\title{
A REDUCTION-PRESERVING COMPLETION FOR PROVING CONFLUENCE OF NON-TERMINATING TERM REWRITING SYSTEMS *
}

\author{
TAKAHITO AOTO AND YOSHIHITO TOYAMA
}

RIEC, Tohoku University, 2-1-1 Katahira, Aoba-ku, Sendai, Miyagi, 980-8577, Japan

e-mail address: \{aoto,toyama\}@nue.riec.tohoku.ac.jp

\begin{abstract}
We give a method to prove confluence of term rewriting systems that contain non-terminating rewrite rules such as commutativity and associativity. Usually, confluence of term rewriting systems containing such rules is proved by treating them as equational term rewriting systems and considering $E$-critical pairs and/or termination modulo $E$. In contrast, our method is based solely on usual critical pairs and it also (partially) works even if the system is not terminating modulo $E$. We first present confluence criteria for term rewriting systems whose rewrite rules can be partitioned into a terminating part and a possibly non-terminating part. We then give a reduction-preserving completion procedure so that the applicability of the criteria is enhanced. In contrast to the well-known KnuthBendix completion procedure which preserves the equivalence relation of the system, our completion procedure preserves the reduction relation of the system, by which confluence of the original system is inferred from that of the completed system.
\end{abstract}

\section{INTRODUCTION}

Confluence is one of the most important properties of term rewriting systems (TRSs for short) and hence many efforts have been spent on developing techniques to prove this property [4, 21. One of the classes of TRSs for which many known confluence proving methods are not effective is the class of TRSs containing associativity and commutativity rules (ACrules). Such TRSs are non-terminating by the existence of AC-rules (more precisely, the commutativity rule is self-looping and associativity rules are looping under the presence of the commutativity rule) and hence the Knuth-Bendix criterion (i.e. terminating TRSs are confluent iff all critical pairs are joinable) does not apply. Furthermore, confluence criteria regardless of termination based on critical pairs often do not apply either.

1998 ACM Subject Classification: D.3.1, F.3.1, F.4.2, I.2.2.

Key words and phrases: Confluence, Completion, Equational Term Rewriting Systems, Confluence Modulo Equations.

* This is a revised and extended version of the paper: Takahito Aoto and Yoshihito Toyama, A ReductionPreserving Completion for Proving Confluence of Non-Terminating Term Rewriting Systems, in Proceedings of the 22nd International Conference on Rewriting Techniques and Applications, LIPIcs, Vol.10, Schloss Dagstuhl - Leibniz-Zentrum fuer Informatik, pp.91-106, 2011. IN COMPUTER SCIENCE
DOI:10.2168/LMCS-8 (1:31) 2012
A REDUCTION-PRESERVING COMPLETION FOR PROVING CONFLUENCE Creative Commons 
A well-known approach to deal with TRSs containing AC-rules is to deal with them as equational term rewriting systems [8, 9, 19]. In this approach, non-terminating rules such as AC-rules are treated exceptionally as an equational subsystem $\mathcal{E}$. Then the confluence of equational term rewriting system $\langle\mathcal{R}, \mathcal{E}\rangle$ is obtained if $\mathcal{R}$ is terminating modulo $\mathcal{E}$ [ 8 , 9, 19, and either the $\mathcal{E}$-critical pairs of $\mathcal{R}$ satisfy certain conditions [9, 19] or $\mathcal{R}$ is left-linear and the $\mathcal{E} / \mathcal{R}$-critical pairs satisfy a certain condition [8]. This approach, however, only works if $\mathcal{R}$ is terminating modulo $\mathcal{E}$. Furthermore, the computation of $\mathcal{E}$-critical pairs requires a finite and complete $\mathcal{E}$-unification algorithm which depends on $\mathcal{E}$.

In this paper, we give a method to prove confluence of TRSs that contain non-terminating rewrite rules such as AC-rules. In contrast to the traditional approach described above, our method is based solely on usual critical pairs and it also (partially) works even if the system is not terminating modulo $\mathcal{E}$. Thus the implementation of the method requires few special ingredients and the method is easily integrated into confluence provers and combined with other confluence proving methods.

Let us explain the idea of our approach via concrete examples.

Example 1.1. Let $\mathcal{R}_{1}$ be the TRS consisting of the commutativity rule and an associativity rule.

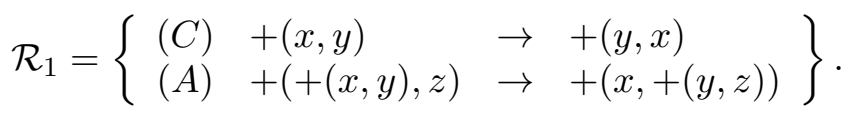

This TRS is non-terminating and many known critical pair conditions for left-linear TRSs do not apply. However, $\mathcal{R}_{1}$ is confluent, i.e. $s \stackrel{*}{\rightarrow} \mathcal{R}_{1} t_{0}$ and $s \stackrel{*}{\rightarrow} \mathcal{R}_{1} t_{1}$ imply $t_{0} \stackrel{*}{\rightarrow}_{\mathcal{R}_{1}} u$ and $t_{1} \stackrel{*}{\rightarrow}_{\mathcal{R}_{1}} u$ for some $u$. Here $s \stackrel{*}{\rightarrow}_{\mathcal{R}_{1}} t$ denotes that $s$ rewrites to $t$ in arbitrary many rewrite steps. One way to prove this is by observing that $\mathcal{R}_{1}$ is reversible, i.e. $s \stackrel{*}{\rightarrow}_{\mathcal{R}_{1}} t$ iff $t \stackrel{*}{\rightarrow}_{\mathcal{R}_{1}} s$. This holds because for any rewrite rule $l \rightarrow r \in \mathcal{R}_{1}$ we have $r \stackrel{*}{\rightarrow}_{\mathcal{R}_{1}} l$ : for the $(C)$-rule, this holds obviously, and for the $(A)$-rule, this holds because $+(x,+(y, z)) \stackrel{*}{\rightarrow}_{\mathcal{R}_{1}}+(+(x, y), z)$ viz.

$$
\begin{aligned}
+(x,+(y, z)) & \rightarrow_{C}+(+(y, z), x) \\
& \rightarrow_{A}+(y,+(z, x)) \\
& \rightarrow_{C}+(+(z, x), y) \\
& \rightarrow_{A}+(z,+(x, y)) \\
& \rightarrow_{C}+(+(x, y), z) .
\end{aligned}
$$

Thus for any $s_{1} \rightarrow \mathcal{R}_{1} s_{2} \rightarrow \mathcal{R}_{1} \cdots \rightarrow \rightarrow_{\mathcal{R}_{1}} s_{n}$, we have $s_{n} \stackrel{*}{\rightarrow}_{\mathcal{R}_{1}} s_{n-1} \stackrel{*}{\rightarrow} \mathcal{R}_{1} \cdots \stackrel{*}{\rightarrow} \mathcal{R}_{1} s_{1}$. Hence $s \stackrel{*}{\rightarrow} \mathcal{R}_{1} t_{0}$ and $s \stackrel{*}{\rightarrow} \mathcal{R}_{1} t_{1}$ imply $t_{0} \stackrel{*}{\rightarrow} \mathcal{R}_{1} s$ and $t_{1} \stackrel{*}{\rightarrow}_{\mathcal{R}_{1}} s$.

Example 1.2. Next we consider the TRS $\mathcal{R}_{2}$, which extends $\mathcal{R}_{1}$ slightly.

$$
\mathcal{R}_{2}=\left\{\begin{array}{lll}
\left(\operatorname{add}_{1}\right) & +(0, y) & \rightarrow y \\
\left(\operatorname{add}_{2}\right) & +(\mathrm{s}(x), y) & \rightarrow \mathrm{s}(+(x, y)) \\
(C) & +(x, y) & \rightarrow+(y, x) \\
(A) & +(+(x, y), z) & \rightarrow+(x,+(y, z))
\end{array}\right\} .
$$

$\mathcal{R}_{2}$ is a TRS consisting of rules for addition of natural numbers denoted by $0, \mathrm{~s}(0), \mathrm{s}(\mathrm{s}(0)), \ldots$ and AC-rules for plus. The TRS $\mathcal{R}_{2}$ is again non-terminating and many known critical pair conditions for left-linear TRSs also do not apply. However, $\mathcal{R}_{2}$ is confluent. This can be explained like this. Since $+(y, 0) \rightarrow_{\mathcal{R}_{2}}+(0, y) \rightarrow_{\mathcal{R}_{2}} y$ and $+(y, \mathrm{~s}(x)) \rightarrow_{\mathcal{R}_{2}}+(\mathrm{s}(x), y) \rightarrow_{\mathcal{R}_{2}}$ $\mathrm{s}(+(x, y))$, together with $\left(\operatorname{add}_{1}\right),\left(\operatorname{add}_{2}\right)$-rules, all occurrences of the symbol 0 in a term can be eliminated and all occurrences of the symbol $\mathrm{s}$ can be moved to the top of the term. 
Hence, for any term $t$, we have $t \stackrel{*}{\rightarrow}_{\mathcal{R}_{2}} \mathrm{~s}^{k}(+(\cdots x \cdots))$ where $k$ is the number of occurrences of the symbol $\mathrm{s}$ in the term $t$ and the part " $+(\cdots x \cdots)$ " denotes the addition of all variables contained in the term $t$. Thus for any $u_{1}, u_{2}$ such that $t \stackrel{*}{\rightarrow}_{\mathcal{R}_{2}} u_{1}$ and $t \stackrel{*}{\rightarrow}_{\mathcal{R}_{2}} u_{2}$, we have $u_{1} \stackrel{*}{\rightarrow}_{\mathcal{R}_{2}} \mathrm{~s}^{k}(+(\cdots x \cdots))$ and $u_{2} \stackrel{*}{\rightarrow}_{\mathcal{R}_{2}} \mathrm{~s}^{k}(+(\cdots x \cdots))$. It remains to use the reversibility of AC-rules (i.e. $\left.\mathcal{R}_{1}\right)$ to join two terms of the form $\mathrm{s}^{k}(+(\cdots x \cdots))$ because they are equivalent modulo associativity and commutativity.

A key point of this method is that, in addition to rewrite rules of $\mathcal{R}_{2}$, we considered auxiliary rewrite rules $\operatorname{add}_{3}:+(y, 0) \rightarrow y$ and $\operatorname{add}_{4}:+(y, \mathrm{~s}(x)) \rightarrow \mathrm{s}(+(x, y))$. In our method, such rewrite rules are added via a reduction-preserving completion procedure. In contrast to the well-known Knuth-Bendix completion procedure which preserves the equivalence relation of the system, our completion procedure preserves the reduction relation of the system, by which confluence of the original system is inferred from that of the completed system. We note that the Knuth-Bendix completion procedure for equational term rewriting systems was initiated by [11, 12, 13, 19] and is generalized in [5, 9]. Since the Knuth-Bendix completion procedure needs to preserve equivalence relation but not necessarily reduction relation, much flexibilities are allowed for the Knuth-Bendix completion procedure compared to our reduction-preserving completion procedure.

The contribution of this paper is summarized as follows:

(1) new abstract criterion for the property Church-Rosser modulo (Theorem 2.2),

(2) new confluence criteria (Theorems 3.8 and 3.28,

(3) reduction-preserving completion for proving confluence and

(4) implementation and experiments for these techniques.

This paper is a revised and extended version of [2]. Compared to [2], Theorems 2.2, 3.8 and 3.28 are new - these extend the results in [2] which are adapted as Corollaries $12.3,3.10$ and 3.29 respectively in the present paper.

The rest of the paper is organized as follows. We first present a criterion for ChurchRosser modulo in an abstract setting (Section 2). Then based on this abstract criterion, we present confluence criteria for TRSs whose rewrite rules can be partitioned into a terminating part and a possibly non-terminating part (Section 3). We then give a reductionpreserving completion procedure so that the applicability of the criteria is enhanced (Section 4). Finally we report on our implementation and results of experiments (Section 5).

\section{Abstract Criterion for Church-Rosser modulo}

In this section, after providing some preliminaries (subsection 1), we present a criterion for Church-Rosser modulo an equivalence relation and present some corollaries of the criterion that have been appeared in the literature (subsection 2). Then we compare our criterion and other abstract criteria for Church-Rosser modulo an equivalence relation (subsection $3)$.

\footnotetext{
${ }^{1}$ Corollaries 2.3 and 3.10 are incorrectly claimed to be original in [2].
} 
2.1. Preliminaries. In this subsection, we fix some notions and notations on relations that will be used throughout the paper.

Let $\rightarrow$ be a relation on a set $A$. The inverse of $\rightarrow$ is denoted by $\leftarrow$. The reflexive closure (the symmetric closure, the transitive closure, the reflexive and transitive closure, the equivalence closure $)$ of $\rightarrow$ is denoted by $\stackrel{\bar{\rightarrow}}{\rightarrow}(\leftrightarrow, \stackrel{+}{\rightarrow}, \stackrel{*}{\rightarrow} \stackrel{*}{\leftrightarrow}$, respectively). We will also use $\rightarrow_{0}, \rightarrow_{1}, \ldots, \Rightarrow, \leadsto, \triangleright, \ldots$ for binary relations, $\mapsto, \bowtie, \ldots$ for symmetric relations and $\sim, \ldots$ for equivalence relations. Closures for such relations are written in the similar way.

The union of two relations $\rightarrow$ and $\Rightarrow$ is written as $\rightarrow \cup \Rightarrow$. For any two relations $\rightarrow$ and $\Rightarrow$, we write $\rightarrow \subseteq \Rightarrow$ if $a \rightarrow b$ implies $a \Rightarrow b$ for any $a, b$. The composition of relations $\rightarrow$ and $\Rightarrow$ is written as $\rightarrow 0 \Rightarrow$. For a (possibly infinite) number of indexed relations $\left(\rightarrow_{\alpha}\right)_{\alpha \in I}$ where $I$ is a set of indexes, $\bigcup_{\alpha \in I} \rightarrow_{\alpha}$ is written as $\rightarrow_{I}$. We will identify element and singleton set in this notation, i.e. $\rightarrow_{\{\alpha\}}=\rightarrow_{\alpha}$. Closures for such relations are written in the similar way.

A relation $\rightarrow$ is well-founded if there exists no infinite descending chain $a_{0} \rightarrow a_{1} \rightarrow \cdots$. The relation $\rightarrow$ is said to be confluent if $\stackrel{*}{\leftarrow} \circ \stackrel{*}{\rightarrow} \subseteq \stackrel{*}{\rightarrow} \circ \stackrel{*}{\leftarrow}$ holds. The relation $\rightarrow$ is said to be Church-Rosser modulo an equivalence relation $\sim(C R M$ in short $)$ if $\stackrel{*}{\bowtie} \subseteq \stackrel{*}{\rightarrow} \circ \sim \circ \stackrel{*}{\leftarrow}$ holds, where $\bowtie=\leftrightarrow \cup \sim$.

2.2. Abstract criterion for Church-Rosser modulo. In this subsection, we give a new criterion for Church-Rosser modulo an equivalence relation.

In what follows, we consider a (strict) partial order $\succ$ on the set $I$ of indexes. Let $I$ be the set of indexes. For a set $J \subseteq I$ of indexes, the set $\{\beta \in I \mid \exists \alpha \in J . \beta \prec \alpha\}$ is written as $\curlyvee J$. If $J=\{\alpha\}$, we write $\curlyvee \alpha$ instead of $\curlyvee\{\alpha\}$. We assume that $\curlyvee$ associates stronger than $\cup$ i.e. $\curlyvee I_{1} \cup \curlyvee I_{2}=\left(\curlyvee I_{1}\right) \cup\left(\curlyvee I_{2}\right)$. The next lemma, which is the basis of our abstract criterion for Church-Rosser modulo, is obtained by induction on the set of indexes w.r.t. the well-founded order $\succ$.

Lemma 2.1. Let I be a set of indexes equipped with a well-founded order $\succ$. Let $\vdash_{\alpha}, \rightarrow_{\alpha}$ be relations on a set $A$ such that $\mapsto_{\alpha}$ is symmetric for each $\alpha \in I$. Let $\Rightarrow_{\alpha}=\mapsto_{\alpha} \cup \rightarrow_{\alpha}$ for each $\alpha \in I$. Suppose (i) $\leftarrow_{\alpha} \circ \rightarrow_{\beta} \subseteq \stackrel{*}{\Leftrightarrow}_{\curlyvee \alpha \cup \curlyvee \beta}$ and (ii) $\mapsto_{\alpha} \circ \rightarrow_{\beta} \subseteq \stackrel{*}{\Leftrightarrow}_{\curlyvee \alpha \cup \curlyvee \beta}$. Then $\rightarrow_{I}$ is Church-Rosser modulo ${ }^{*}{ }_{I}$.

Proof. For each sequence $a_{0} \Leftrightarrow_{\alpha_{0}} a_{1} \Leftrightarrow_{\alpha_{1}} \cdots \Leftrightarrow_{\alpha_{n-1}} a_{n}$, let its weight be the multiset consisting of the indexes of the each steps i.e. $\left\{\alpha_{0}, \alpha_{1}, \ldots, \alpha_{n-1}\right\}$. Let $\gg$ be the multiset extension of the well-founded order $\succ$. We show by well-founded induction on the weight of the sequence w.r.t. $\gg$ that for any sequence $a_{0} \stackrel{*}{\Leftrightarrow} I a_{n}$ there exists a sequence $\left.a_{0} \stackrel{*}{\rightarrow}_{I} \circ \vdash^{*}\right|_{I}$ $\circ \stackrel{*}{\leftarrow} I a_{n}$.

(1) Suppose there exists $k$ such that $a_{k-1} \leftarrow_{\alpha} a_{k} \rightarrow_{\beta} a_{k+1}$. Then by assumption (i), there exists a sequence $a_{k-1} \stackrel{*}{\Leftrightarrow} \curlyvee \alpha \cup \curlyvee \beta a_{k+1}$. Thus we have a sequence $a_{0} \stackrel{*}{\Leftrightarrow}_{I} a_{k-1} \stackrel{*}{\Leftrightarrow} \curlyvee \alpha \cup \curlyvee \beta$ $a_{k+1} \stackrel{*}{\Leftrightarrow} I a_{n}$. Since this new sequence has a weight less than the original sequence $a_{0} \stackrel{*^{*}}{*} a_{n}$, it follows that there exists a sequence $a_{0} \stackrel{*}{\rightarrow}_{I} \circ{\stackrel{*}{H}}_{I} \circ \stackrel{*^{*}}{\leftarrow} a_{n}$ by the induction hypothesis.

(2) Suppose that there exists $k$ such that $a_{k-1} \vdash_{\alpha} a_{k} \rightarrow_{\beta} a_{k+1}$. Then by assumption (ii), there exists a sequence $a_{k-1} \stackrel{*}{\Leftrightarrow} \curlyvee \alpha \cup \curlyvee \beta a_{k+1}$. Thus, it follows that there exists a sequence $a_{0} \stackrel{*}{\rightarrow} I \circ \stackrel{*}{H}_{I} \circ \stackrel{*}{\leftarrow} I a_{n}$ by the induction hypothesis as in the previous case. 
(3) Suppose that there exists $k$ such that $a_{k-1} \leftarrow_{\alpha} a_{k} \mapsto_{\beta} a_{k+1}$. Then one can show that there exists a sequence $a_{0} \stackrel{*}{\rightarrow}_{I} \circ \stackrel{*}{H}_{I} \circ \stackrel{*}{\leftarrow}_{I} a_{n}$ in the same way as the case (2).

(4) It remains to treat the case that $(\alpha)$ there exists no $k$ such that $a_{k-1} \leftarrow \leftarrow_{I} a_{k} \rightarrow_{I} a_{k+1}$, $(\beta)$ there exists no $k$ such that $a_{k-1} \mapsto_{I} a_{k} \rightarrow_{I} a_{k+1}$ and $(\gamma)$ there exists no $k$ such that $a_{k-1} \leftarrow_{I} a_{k} \mapsto_{I} a_{k+1}$. We show by induction on the length of $a_{0} \stackrel{*}{\Leftrightarrow} a_{n}$ that this sequence has the form $a_{0} \stackrel{*}{\rightarrow}_{I} \circ \stackrel{*}{H}_{I} \circ \stackrel{*}{\leftarrow}_{I} a_{n}$. The case $n=0$ is trivial. Suppose $a_{0} \Leftrightarrow_{I} a_{1} \stackrel{*}{\Leftrightarrow} I a_{n}$. By induction hypothesis we have $a_{1} \stackrel{*}{\rightarrow}_{I} a_{l} \stackrel{*}{\mapsto}_{I} a_{m} \stackrel{*^{*}}{\leftarrow} a_{n}$ for some $1 \leq l, m \leq n$. We distinguish three cases:

(a) $a_{0} \mapsto_{I} a_{1}$. By $(\beta)$, it follows that we have $a_{0} \dashv_{I} a_{1}=a_{l} \mapsto^{*}{ }_{I} a_{m} \stackrel{*}{\leftarrow} I a_{n}$. Hence the conclusion follows.

(b) $a_{0} \rightarrow_{I} a_{1}$. Since we have $a_{0} \rightarrow_{I} a_{1} \stackrel{*}{\rightarrow} I a_{l} \mapsto^{*}{ }_{I} a_{m} \stackrel{*}{\leftarrow} I a_{n}$, the conclusion follows.

(c) $a_{0} \leftarrow \leftarrow_{I} a_{1}$. Then by $(\alpha)$, it follows that we have $a_{0} \leftarrow \leftarrow_{I} a_{1}=a_{l} \stackrel{*}{\vdash}_{I} a_{m} \stackrel{*}{\leftarrow} I a_{n}$. Furthermore, by $(\gamma)$, it follows that $a_{0} \leftarrow_{I} a_{1}=a_{l}=a_{m} \stackrel{*}{\leftarrow} I a_{n}$. Hence the conclusion follows.

The following abstract criterion for Church-Rosser modulo will be used as the basis of all of our confluence criteria presented in this paper.

Theorem 2.2 (abstract criteria for CRM). Let $\mapsto, \rightarrow, \sim$ be relations on a set $A$ such that $\mapsto$ is symmetric, $\leadsto \subseteq \mapsto$, and $\rightarrow \circ \stackrel{*}{\sim}$ is well-founded. Let $\Rightarrow=\leadsto \cup \rightarrow$. Suppose

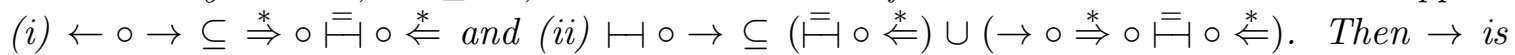
Church-Rosser modulo ${ }^{*}$.

Proof. Let $\rightarrow=\mapsto \cup \rightarrow$. Suppose that the index of a step $a \rightarrow b$ be given by the multiset $l a b(a \rightarrow b)$ defined like this: $\operatorname{lab}(a \mapsto b)=\{a, b\}$ (i.e. $\operatorname{la} b(a \leadsto b)=\{a, b\}), \operatorname{la}(a \rightarrow b)=$ $\{a\}$. Let $\succ$ be the multiset extension of the transitive closure of the well-founded relation $\rightarrow \circ \stackrel{*}{\rightarrow}$. Then by our assumption it readily follows that (i) $\leftarrow_{\alpha} \circ \rightarrow_{\beta} \subseteq \stackrel{*}{\rightarrow} \triangleright_{\curlyvee \alpha \cup \curlyvee \beta}$ and (ii) $\mapsto_{\alpha} \circ \rightarrow_{\beta} \subseteq \stackrel{*}{\leftrightarrow} \triangleright_{\curlyvee \alpha \cup \curlyvee \beta}$ are satisfied. Thus, from Lemma 2.1. $\rightarrow$ is Church-Rosser modulo $\stackrel{*}{*}$.

Several corollaries of the theorem follow.

Corollary 2.3 (Corollary of Propositions 1 and 3 of [10]). Let $\mapsto, \rightarrow$ be relations on a set A such that $\mapsto$ is symmetric and $\rightarrow$ is well-founded. Suppose $(i) \leftarrow \circ \rightarrow \subseteq \stackrel{*}{\rightarrow} \circ \models^{*} \circ \stackrel{*}{\leftarrow}$ and (ii) $\mapsto \circ \rightarrow \subseteq \stackrel{*}{\rightarrow} \circ \stackrel{\models}{\models} \circ \stackrel{*}{\leftarrow}$. Then $\rightarrow$ is Church-Rosser modulo $\vdash^{*}$.

Proof. Take $\leadsto:=\emptyset$ in Theorem 2.2 .

Corollary 2.4. Let $\mapsto, \rightarrow, \leadsto$ be relations on a set $A$ such that $\mapsto$ is symmetric, $\leadsto \subseteq \mapsto$, and $\rightarrow \circ \stackrel{*}{\sim}$ is well-founded. Let $\Rightarrow=\rightarrow \cup \leadsto$. Suppose (i) $\leftarrow \circ \rightarrow \subseteq \stackrel{*}{\Rightarrow} \circ \vdash \circ \stackrel{*}{\models}$ and (ii) $\mapsto \circ \rightarrow \subseteq \rightarrow \circ \stackrel{*}{\Rightarrow} \circ \vdash^{=} \circ \stackrel{*}{\models}$. Then $\rightarrow$ is Church-Rosser modulo $\vdash^{*}$.

Proof. Take the case $\mapsto \circ \rightarrow \subseteq \rightarrow \circ \stackrel{*}{\Rightarrow} \circ \stackrel{=}{\models} \circ \stackrel{*}{\models}$ for the condition (ii) in Theorem 2.2

In case $\leadsto:=\mapsto$, necessary and sufficient conditions for CRM are obtained.

Corollary 2.5. Let $\mapsto, \rightarrow$ be relations on a set $A$ such that $\mapsto$ is symmetric and $\rightarrow \circ \mapsto^{*}$ is well-founded. Let $\Rightarrow=\rightarrow \cup \mapsto$. Then $\rightarrow$ is Church-Rosser modulo ${ }^{*}$ if and only if $(i)$ $\leftarrow \circ \rightarrow \subseteq \stackrel{*}{\Rightarrow} \circ \stackrel{*}{\Leftarrow}$ and $(i i) \mapsto \circ \rightarrow \subseteq \rightarrow \circ \stackrel{*}{\Rightarrow} \circ \stackrel{*}{\Leftarrow}$. 


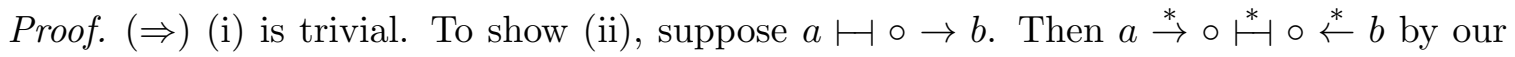
assumption. Since $\stackrel{+}{\rightarrow} \circ \stackrel{*}{\vdash}^{\circ} \circ \stackrel{*}{\leftarrow} \subseteq \rightarrow \circ \stackrel{*}{\Rightarrow} \circ \stackrel{*}{\leftarrow}$, it remains to exclude the case $a \stackrel{*}{*} \circ \stackrel{*}{\leftarrow} b$. If $a \stackrel{*}{\vdash} \circ \stackrel{*}{\leftarrow} b$ then $a \mapsto \circ \rightarrow b \stackrel{*}{\rightarrow} \circ \stackrel{*}{\vdash}^{\prime} a$. This contradicts our assumption that $\rightarrow \circ \vdash^{*}$ is well-founded. $(\Leftarrow)$ follows from Corollary 2.4 .

The conditions (i), (ii) can be replaced with particular forms.

Corollary 2.6. Let $\mapsto, \rightarrow$ be relations on a set $A$ such that $\mapsto$ is symmetric and $\rightarrow \circ \mapsto^{*}$ is

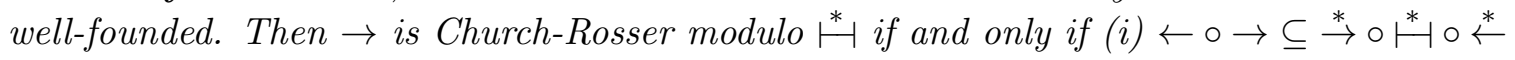
and (ii) $\mapsto \circ \rightarrow \subseteq \stackrel{+}{\rightarrow} \circ \vdash^{*} \circ \stackrel{*}{\leftarrow}$.

Proof. $(\Rightarrow)$ (i) is trivial. To show (ii), suppose $a \mapsto \circ \rightarrow b$. Then $a \stackrel{*}{\rightarrow} \circ \vdash^{*} \circ \stackrel{*}{\leftarrow} b$ by our assumption. Thus it remains to exclude the case $a \stackrel{*}{\mapsto} \circ \stackrel{*}{\leftarrow} b$. If $a \vdash^{*} \circ \stackrel{*}{\leftarrow} b$ then

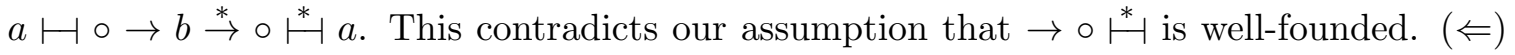
Let $\Rightarrow=\rightarrow \cup \mapsto$. Then $\leftarrow \circ \rightarrow \subseteq \stackrel{*}{\rightarrow} \circ \vdash^{*} \circ \circ \stackrel{*}{\leftarrow} \subseteq \stackrel{*}{\Rightarrow} \circ \stackrel{*}{\leftarrow}$ and $\mapsto \circ \rightarrow \subseteq \stackrel{+}{\rightarrow} \circ \vdash^{*} \circ \stackrel{*}{\leftarrow} \subseteq \rightarrow \circ \stackrel{*}{\Rightarrow} \circ \stackrel{*}{\leftarrow}$. Hence the claim follows from Corollary 2.5.

Remark 2.7. Note that since Corollaries 2.5 and 2.6 give the necessary and sufficient conditions for CRM, the conditions (i), (ii) of Corollary 2.5 imply the conditions (i), (ii) of Corollary 2.6.

The condition (ii) $\mapsto \circ \rightarrow \subseteq \stackrel{+}{\rightarrow} \circ \vdash^{*} \circ \stackrel{*}{\leftarrow}$ in this corollary can be replaced with (ii) $\mapsto \circ \rightarrow \subseteq \stackrel{*}{\rightarrow} \circ \vdash^{*} \circ \stackrel{*}{\leftarrow}$ as shown in the next corollary.

Corollary 2.8 (Lemma 2.8 of [8]). Let $\mapsto, \rightarrow$ be relations on a set $A$ such that $\mapsto$ is symmetric and $\rightarrow \circ{ }^{*}$ is well-founded. Then $\rightarrow$ is Church-Rosser modulo ${ }^{*}$ if and only if (i) $\leftarrow \circ \rightarrow \subseteq \stackrel{*}{\rightarrow} \circ \vdash^{*} \circ \stackrel{*}{\leftarrow}$ and $($ ii $) \mapsto \circ \rightarrow \subseteq \stackrel{*}{\rightarrow} \circ \vdash^{*} \circ \stackrel{*}{\leftarrow}$.

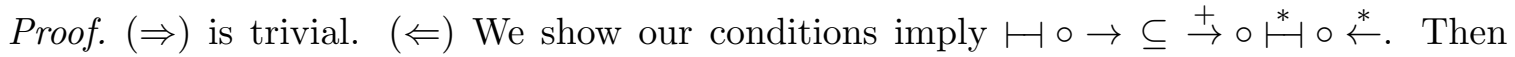
the claim follows from Corollary 2.6. Suppose contrarily that we have $a \mapsto \circ \rightarrow b$ but not $a \stackrel{+}{\rightarrow} \circ \vdash^{*} \circ \stackrel{*}{\leftarrow} b$. From $a \mapsto \circ \rightarrow b$, we have $a \stackrel{*}{\rightarrow} \circ \vdash^{*} \circ \stackrel{*}{\leftarrow} b$ by our assumption. Hence $a \stackrel{*}{\vdash} \circ \stackrel{*}{\leftarrow} b$ holds. Thus $a \mapsto \circ \rightarrow b \stackrel{*}{\rightarrow} \circ \vdash^{*} a$. This contradicts our assumption that $\rightarrow \circ \vdash^{*}$ is well-founded.

Remark 2.9. The proof of Corollary 2.8 given in $[8]$ is based on a combinatorial argument; another proof of Corollary 2.8 based on an argument similar to the proof of Lemma 2.1 has been given in [14].

We now give a proof of Theorem 5 of [9] based on Lemma 2.1.

Lemma 2.10. Let $\mapsto, \rightarrow$ be relations on a set $A$ such that $\mapsto$ is symmetric and $\rightarrow \circ \mapsto^{*}$ is well-founded. Let be a relation on $A$ satisfying $\rightarrow \subseteq \backslash \subseteq \vdash^{*} \circ \rightarrow \circ \vdash^{*}$. Then $\downarrow$ is

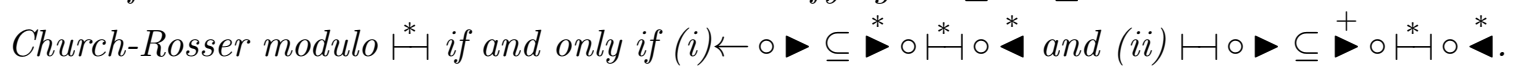

Proof. $\left(\Rightarrow\right.$ ) (i) is trivial. (ii) Suppose $a \longmapsto \circ \diamond b$. Then $a \stackrel{*}{\bullet^{*}} \circ \stackrel{*}{\bullet}^{*} b$ by our assumption. Thus it remains to exclude the case $a \vdash^{*} \circ \bullet^{*} b$. If $a \vdash^{*} \circ \bullet^{*} b$ then $a \mapsto \circ \bullet b \stackrel{*}{\bullet} \circ \vdash^{*} a$. Hence $\left.a\left(\vdash^{*} \circ \rightarrow \circ \vdash^{*}\right)\right)^{+} a$. This contradicts our assumption that $\rightarrow \circ \vdash^{*}$ is well-founded. $(\Leftarrow)$ By assumption, $\vdash^{*} \circ \rightarrow \circ \vdash^{*}$ is well-founded. Let $\triangleright=\left(\vdash^{*} \circ \rightarrow \circ \vdash^{*} \mid\right)^{+}$. Clearly, $\triangleright$ is well-founded. Note that $a \vdash^{*} \circ \circ \vdash^{*} b$ implies $a \triangleright b$. Let $\Rightarrow=\triangleright \cup \mapsto$. For each step $a \Leftrightarrow b$, let its index be given by the multiset $l a b(a \mapsto b)=\{a, b\}, l a b(a-b)=\{a\}$ and 
$l a b(a \triangleleft b)=\{b\}$. Let $\succ$ be the multiset extension of $\triangleright$. Then, $\succ$ is a well-founded relation on the set of indexes. By Lemma 2.1. it suffices to show (i') $\boldsymbol{\triangleleft}_{\alpha} \circ \boldsymbol{}_{\beta} \subseteq \stackrel{*}{\Leftrightarrow}_{\curlyvee \alpha \cup \curlyvee \beta}$ and (ii') $\mapsto_{\alpha} \circ \boldsymbol{D}_{\beta} \subseteq \stackrel{*}{\Leftrightarrow}_{\curlyvee \alpha \cup \curlyvee \beta}$.

To prove (i'), we claim that for any $b \triangleleft a=a_{0} \mapsto a_{1} \mapsto \cdots \mapsto a_{m} \rightarrow c^{\prime} \mapsto^{*} c$, we have $b \stackrel{*}{\Leftrightarrow}_{\curlyvee\{a\}} c$. As $\alpha=\beta=\{a\},\left(\mathrm{i}^{\prime}\right)$ immediately follows from this claim. Our proof proceeds by induction on $m$.

- Suppose $m=0$. Then we have $b \triangleleft a \rightarrow c^{\prime} \vdash^{*} c$. Then, by our condition (i), $b \stackrel{*}{\vdash} \circ \vdash^{*} \circ \bullet^{*}$ $c^{\prime} \vdash^{*} \mid c$. Since $b \triangleleft a$ and $a \triangleright c^{\prime}$, we have $b \stackrel{*}{\Leftrightarrow} \curlyvee\{a\} c$.

- Suppose $m>0$. Then, since we have $b \triangleleft a \mapsto a_{1}$, by our condition (ii), $b \stackrel{*}{\bullet} \circ \vdash^{*} \circ \bullet^{*}$ $a_{1}^{\prime} \triangleleft a_{1}$ for some $a_{1}^{\prime}$. Then we have $a_{1}^{\prime} \triangleleft a_{1} \mapsto \cdots \mapsto a_{m} \rightarrow c^{\prime} \mapsto^{*} c$, and hence, by induction hypothesis, $a_{1}^{\prime}{\stackrel{*}{\Leftrightarrow} \curlyvee\left\{a_{1}\right\}} c$. Since $a \mapsto a_{1}, a_{1} \triangleright d$ implies $a \triangleright d$ for any $d$. Hence

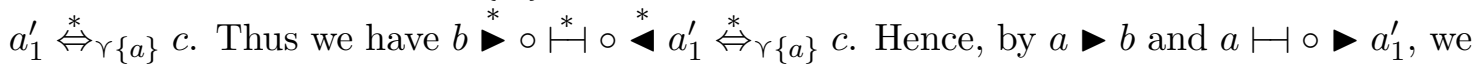
have $b \stackrel{*}{\Leftrightarrow} \curlyvee\{a\} c$.

To prove (ii'), we claim that for any $b \mapsto a \triangleright c$, we have $b \stackrel{*}{\Leftrightarrow} \curlyvee\{a, b\} c$. From the condition (ii) we have $b \triangleright b^{\prime} \stackrel{*}{\bullet} \circ \vdash^{*} \circ \stackrel{*}{\hookrightarrow} c$. By $a \mapsto b \triangleright b^{\prime}$ and $a \triangleright c$ we have $b^{\prime} \stackrel{*}{\Leftrightarrow} \curlyvee\{a\} c$, and by $b \triangleright b^{\prime}$ we have $b \stackrel{*}{\Leftrightarrow} \curlyvee\{a, b\} b^{\prime}$. Hence, we obtain $b \stackrel{*}{\Leftrightarrow} \curlyvee\{a, b\} c$.

Thus, from Lemma 2.1, we conclude $\rightarrow$ is Church-Rosser modulo ${ }^{*}$.

Let be a relation on a set $A$ satisfying $\rightarrow \subseteq \triangleright \wedge^{*} \circ \rightarrow \circ{ }^{*}$. Then $\rightarrow$ is said to be -Church-Rosser modulo $\vdash^{*}$ if $\stackrel{*}{\bowtie} \subseteq \stackrel{*}{\bullet} \circ \vdash^{*} \circ \stackrel{*}{\triangleleft}$, where $\bowtie=\leftrightarrow \cup \mapsto$.

Corollary 2.11 (Theorem 5 of [9]). Let $\mapsto, \rightarrow$ be relations on a set $A$ such that $\mapsto$ is symmetric and $\rightarrow \circ \vdash^{*}$ is well-founded. Let be a relation on $A$ satisfying $\rightarrow \subseteq \triangleright \subseteq$

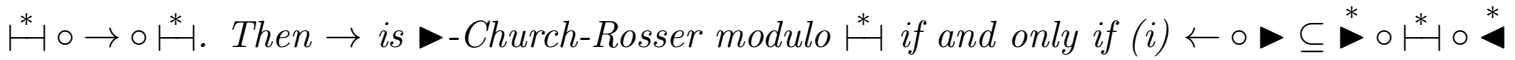

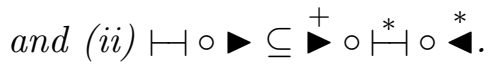

Proof. Let $\bowtie=\leftrightarrow \cup \mapsto$ and $\Rightarrow=\triangleright \cup \mapsto$. From $\rightarrow \subseteq \triangleright \subseteq \vdash^{*} \circ \rightarrow \circ \vdash^{*}$, it follows that $\bowtie \subseteq \Leftrightarrow \subseteq \stackrel{*}{\bowtie}$. Hence, we have $\stackrel{*}{\bowtie}=\stackrel{*}{\Leftrightarrow}$. Thus, from Lemma 2.10, the claim follows.

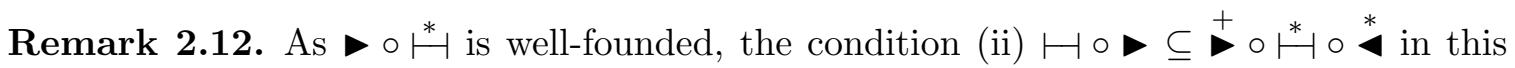

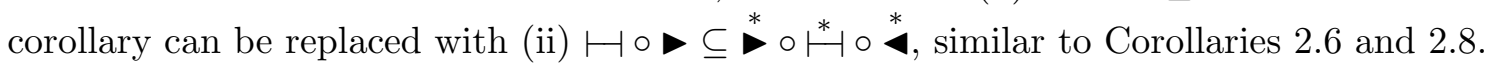

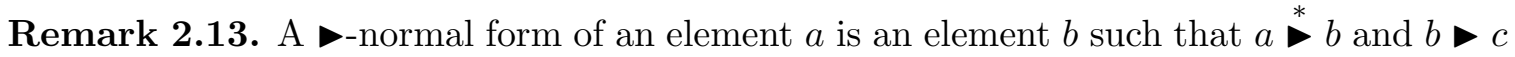
for no $c$. The condition "(i) and (ii)" can be replaced with the condition that, for any $a, b$ and their respective -normal forms $\hat{a}, \hat{b},\left(\mathrm{i}^{\prime}\right) a \leftarrow \circ-b$ implies $\hat{a} \vdash^{*} \hat{b}$ and (ii') $a \mapsto \circ>b$ implies $\hat{a} \vdash^{*} \hat{b}$, which is explained as follows. Clearly, (i') and (ii') imply (i) and (ii). To show the reverse direction, suppose (i) and (ii). Let $\hat{a}, \hat{b}$ be -normal forms $a, b$, respectively.

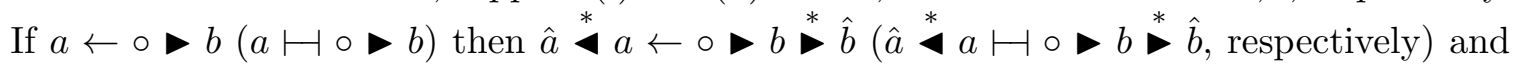
thus $\hat{a}{ }^{*} \hat{b}$, where $\bowtie=\leftrightarrow \cup \mapsto$. Thus $\hat{a} \stackrel{*}{\bullet} \vdash^{*} \circ \bullet^{*} \hat{b}$, as $\rightarrow$ is $\triangleright$-Church-Rosser modulo $\vdash^{*}$. Since $\hat{a}, \hat{b}$ are -normal forms, we conclude $\hat{a} \vdash^{*} \hat{b}$. 
2.3. Related works. Several other abstract criteria for CRM have been obtained in [8] and [15]. In this subsection, we compare our criterion with these.

The following necessary and sufficient criterion for CRM is obtained in [8].

Proposition 2.14 (Corollary of Lemmas 2.6 and 2.7 of [8]). Let $\mapsto, \rightarrow$ be relations on a set $A$ such that $\mapsto$ is symmetric and $\rightarrow$ is well-founded. Then $\rightarrow$ is Church-Rosser modulo ${ }^{*}$ if and only if $\left(i^{\prime}\right) \leftarrow \circ \rightarrow \subseteq \stackrel{*}{\rightarrow} \circ \vdash^{*} \circ \stackrel{*}{\leftarrow}$ and $\left(i i^{\prime}\right){ }^{*} \circ \rightarrow \subseteq \stackrel{*}{\rightarrow} \circ \vdash^{*} \circ \stackrel{*}{\leftarrow}$.

In this proposition, $\rightarrow$ is supposed to be well-founded, similarly to Corollary 2.3. This proposition and Corollary 2.3, however, differ in the following points.

(1) Proposition 2.14 gives necessary and sufficient conditions for CRM, while Corollary 2.3 gives only sufficient conditions.

(2) The condition part of $\left(i i^{\prime}\right)$ of Proposition 2.14 is not localized (i.e. $\vdash^{*} \circ \rightarrow$ is assumed), while that of $(i i)$ of Corollary 2.3 is localized (i.e. $\mapsto \circ \rightarrow$ is assumed).

(3) In the conclusion parts of $\left(i^{\prime}\right)$ and $\left(i i^{\prime}\right)$ of Proposition 2.14 an arbitrary number of $\vdash-$ steps are allowed, while in those of $(i)$ and $(i i)$ of Corollary 2.3 the number of $\mapsto$-steps needs to be at most one.

The decreasing diagram technique [24] is a powerful technique to obtain many confluence criteria. In [15], the technique is extended to obtain a criterion for CRM.

Proposition 2.15 (Theorem 14 of [15]). Let I be a set of indexes equipped with a wellfounded order $\succ$. Let $\rightarrow_{\alpha}$ be a relation on a set $A$ for each $\alpha \in I$ and $\mapsto$ a symmetric

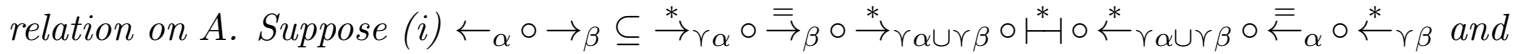

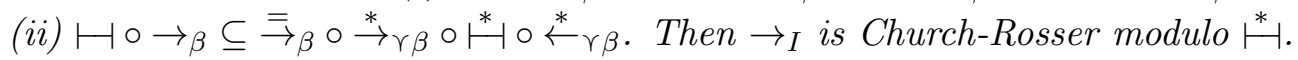

This proposition is given in terms of indexed relations as in Lemma 2.1. This proposition and Lemma 2.1 differ in the following points.

(1) In Lemma 2.1 $\vdash$-steps are indexed, while in Proposition $2.15 \mapsto$-steps are not indexed.

(2) In the case $\succ=\emptyset$, Lemma 2.1 is meaningless, while Proposition 2.15 still remains as a simple criterion for CRM.

Remark 2.16. The labelings like $l a b(a \rightarrow b)=\{a\}$ and $l a b(a \rightarrow b)=\{a, b\}$ used in the proof of Theorem 2.2 are called source and step labelings in [26], which are used to obtain abstract confluence criteria from the decreasing diagram criterion.

\section{Confluence CRiteria}

In this section, we develop several confluence criteria for TRSs that can be partitioned into terminating TRS $\mathcal{S}$ and reversible TRS $\mathcal{P}$. After the preliminaries (subsection 1), we present our first criterion that works for the case that $\mathcal{S}$ is linear (subsection 2). Next we claim another criterion effective for left-linear $\mathcal{S}$ - for this, we first give a criterion using the usual notion of the critical pairs (subsection 3) and then extend the criterion using the notion of parallel critical pairs (subsection 4). Finally, we give some examples and describe relations among the given criteria (subsection 5). 
3.1. Preliminaries. In this subsection, we fix some notions and notations on term rewriting systems and after that we present some lemmas that will be used in later subsections.

Let $\mathcal{F}$ be a set of fixed arity function symbols and $\mathcal{V}$ be a set of variables. The set $\mathrm{T}(\mathcal{F}, \mathcal{V})$ of terms over $\mathcal{F}$ and $\mathcal{V}$ is defined like this: (1) $\mathcal{V} \subseteq \mathrm{T}(\mathcal{F}, \mathcal{V}) ;(2)$ if $f \in \mathcal{F}$ has arity $n$ and $t_{1}, \ldots, t_{n} \in \mathrm{T}(\mathcal{F}, \mathcal{V})$ then $f\left(t_{1}, \ldots, t_{n}\right) \in \mathrm{T}(\mathcal{F}, \mathcal{V})$. The sets of function symbols and variables occurring in a term $t$ are denoted by $\mathcal{F}(t)$ and $\mathcal{V}(t)$, respectively. A linear term is a term in which any variable occurs at most once. Positions are finite sequences of positive integers. The empty sequence is denoted by $\epsilon$. The set of positions in a term $t$ is denoted by $\operatorname{Pos}(t)$. The concatenation of positions $p, q$ is denoted by $p . q$. We use $\leq$ for prefix ordering on positions, i.e. $p \leq q$ iff $\exists o$. $p . o=q$. We write $p<q$ iff $p \leq q$ and $p \neq q$. For positions $p, q$ such that $p \leq q$, the position $o$ satisfying $p . o=q$ is denoted by $q / p$. Positions $p_{1}, \ldots, p_{n}$ are parallel if $p_{i} \not \leq p_{j}$ for any $i \neq j$. We write $p \| q$ if two positions $p, q$ are parallel. For sets $U, V$ of positions, we write $U \| V$ if $p \| q$ holds for any $p \in U$ and $q \in V$. If $p$ is a position in a term $t$, then the symbol in $t$ at the position $p$ is written as $t(p)$, the subterm of $t$ at the position $p$ is written as $t / p$, and the term obtained by replacing the subterm $t / p$ by a term $s$ is written as $t[s]_{p}$. For any $P \subseteq \operatorname{Pos}(t)$, we define $\mathcal{V}_{P}(t)=\bigcup_{p \in P} \mathcal{V}(t / p)$. For $X \subseteq \mathcal{F} \cup \mathcal{V}$, we put $\operatorname{Pos}_{X}(t)=\{p \in \operatorname{Pos}(t) \mid t(p) \in X\}$. For parallel positions $p_{1}, \ldots, p_{n}$ in a term $t$, the term obtained by replacing each subterm $t / p_{i}$ by a term $s_{i}$ is written as $t\left[s_{1}, \ldots, s_{n}\right]_{p_{1}, \ldots, p_{n}}$. A context is an expression $t[, \ldots,]_{p_{1}, \ldots, p_{n}}$ in which such subterms are dropped.

A map $\sigma$ from $\mathcal{V}$ to $\mathrm{T}(\mathcal{F}, \mathcal{V})$ is a substitution if the domain $\operatorname{dom}(\sigma)$ of $\sigma$ is finite where $\operatorname{dom}(\sigma)=\{x \in \mathcal{V} \mid \sigma(x) \neq x\}$. As usual, we identify each substitution with its homomorphic extension. For a substitution $\sigma$ and a term $t, \sigma(t)$ is also written as $t \sigma$. A relation $R$ on $\mathrm{T}(\mathcal{F}, \mathcal{V})$ is stable if for any terms $s, t \in \mathrm{T}(\mathcal{F}, \mathcal{V}), s R t$ implies $s \theta R t \theta$ for any substitution $\theta$; it is monotone if $s R t$ implies $f(\ldots, s, \ldots) R f(\ldots, t, \ldots)$ for any $f \in \mathcal{F}$. A relation $R$ on $\mathrm{T}(\mathcal{F}, \mathcal{V})$ is a rewrite relation if it is stable and monotone.

For a set $\mathcal{E}$ of equations, we write $\mathcal{E}^{-1}=\{r \approx l \mid l \approx r \in \mathcal{E}\}$. Equations are identified modulo renaming (of variables), for example, $+(x, y)=+(y, x)$ equals to $+(y, z)=+(z, y)$. A set $\mathcal{E}=\left\{s_{1} \approx t_{1}, \ldots, s_{n} \approx t_{n}\right\}$ of equations is unifiable if there exists a substitution $\sigma$ such that $s_{i} \sigma=t_{i} \sigma$ for all $i$; the substitution $\sigma$ is a unifier of $\mathcal{E}$. The most general unifier is abbreviated as mgu. If there exists an equation $l \approx r \in \mathcal{E}$ or $r \approx l \in \mathcal{E}$ and a position $p$ in a term $s$ and substitution $\theta$ such that $s / p=l \theta$ and $t=s[r \theta]_{p}$, then we write $s \leftrightarrow_{\mathcal{E}} t$.

An equation $l \approx r$ is a rewrite rule if it satisfies the conditions (1) $l \notin \mathcal{V}$ and (2) $\mathcal{V}(r) \subseteq \mathcal{V}(l)$. A rewrite rule $l \approx r$ is written as $l \rightarrow r$. A rewrite rule $l \rightarrow r$ is linear (left-linear) if $l$ and $r$ are linear ( $l$ is linear, respectively); it is bidirectional if $r \approx l$ is a rewrite rule. A term rewriting system (TRS for short) is a finite set of rewrite rules. A TRS is left-linear (linear, bidirectional) if so are all its rewrite rules. If a TRS $\mathcal{R}$ is bidirectional then $\mathcal{R}^{-1}=\{r \rightarrow l \mid l \rightarrow r \in \mathcal{R}\}$ is a TRS and $\mathcal{R} \cup \mathcal{R}^{-1}$ is a bidirectional TRS. Let $\mathcal{R}$ be a TRS. If there exists a rewrite rule $l \rightarrow r \in \mathcal{R}$ and a position $p$ in a term $s$ and substitution $\theta$ such that $s / p=l \theta$ and $t=s[r \theta]_{p}$, we write $s \rightarrow_{p, \mathcal{R}} t$. If $p(p$ and $\mathcal{R})$ is clear from the context, $s \rightarrow_{p, \mathcal{R}} t$ is written as $s \rightarrow_{\mathcal{R}} t$ ( $s \rightarrow t$, respectively). We call $s \rightarrow_{p, \mathcal{R}} t$ a rewrite step; the subterm $s / p$ is the redex of this rewrite step. We say $t$ is obtained by contracting the redex $s / p$. The relation $\rightarrow_{\mathcal{R}}$ on $\mathrm{T}(\mathcal{F}, \mathcal{V})$ is a rewrite relation and called the rewrite relation of $\mathcal{R}$. A term $s$ is normal if $s \rightarrow_{\mathcal{R}} t$ for no term $t$. The set of normal terms is denoted by $\operatorname{NF}(\mathcal{R})$. A normal form (or $\mathcal{R}$-normal form) of a term $s$ is a term $t \in \mathrm{NF}(\mathcal{R})$ such that $s \stackrel{*}{\rightarrow}_{\mathcal{R}} t$. Two terms $s$ and $t$ are said to be joinable if $s \stackrel{*}{\rightarrow}_{\mathcal{R}} \circ \stackrel{*}{\leftarrow}_{\mathcal{R}} t$. A TRS $\mathcal{R}$ is terminating if $\rightarrow_{\mathcal{R}}$ is well-founded; $\mathcal{R}$ is confluent if $\rightarrow_{\mathcal{R}}$ is confluent. A TRS $\mathcal{R}$ is 
terminating relative to a TRS $\mathcal{P}$ if $\rightarrow_{\mathcal{R}} \circ \stackrel{*}{\rightarrow}_{\mathcal{P}}$ is well-founded; A TRS $\mathcal{R}$ is terminating modulo a set $\mathcal{E}$ of equations if $\rightarrow_{\mathcal{R}} \circ \stackrel{*}{\leftrightarrow} \mathcal{E}$ is well-founded.

Let $s, t$ be terms whose variables are disjoint. The term $s$ overlaps on $t$ (at a position $p$ ) when there exists a non-variable subterm $u=t / p$ of $t$ such that $u$ and $s$ are unifiable. Let $l_{1} \rightarrow r_{1}$ and $l_{2} \rightarrow r_{2}$ be rewrite rules. W.l.o.g. let their variables be disjoint. Suppose that $l_{1}$ overlaps on $l_{2}$ at a position $p$ and $\sigma$ is the mgu of $l_{1}$ and $l_{2} / p$. Then the term $l_{2}\left[l_{1}\right]_{p} \sigma$ yields a critical pair $\left\langle l_{2}\left[r_{1}\right]_{p} \sigma, r_{2} \sigma\right\rangle$ obtained by the overlap of $l_{1} \rightarrow r_{1}$ on $l_{2} \rightarrow r_{2}$ at the position $p$. In the case of self-overlap (i.e. when $l_{1} \rightarrow r_{1}$ and $l_{2} \rightarrow r_{2}$ are identical modulo renaming), we do not consider the case $p=\epsilon$. We call the critical pair outer if $p=\epsilon$ and inner if $p>\epsilon$. The set of outer (inner) critical pairs obtained by the overlaps of a rewrite rule from $\mathcal{R}$ on a rewrite rule from $\mathcal{Q}$ is denoted by $\mathrm{CP}_{\text {out }}(\mathcal{R}, \mathcal{Q})\left(\mathrm{CP}_{\text {in }}(\mathcal{R}, \mathcal{Q})\right.$, respectively). We put $\mathrm{CP}(\mathcal{R}, \mathcal{Q})=\mathrm{CP}_{\text {out }}(\mathcal{R}, \mathcal{Q}) \cup \mathrm{CP}_{\text {in }}(\mathcal{R}, \mathcal{Q})$. For a set $C$ of pairs of terms, we write $C^{-1}=\{\langle v, u\rangle \mid\langle u, v\rangle \in C\}$. We note that $\mathrm{CP}_{\text {out }}(\mathcal{R}, \mathcal{Q})=\mathrm{CP}_{\text {out }}(\mathcal{Q}, \mathcal{R})^{-1}$.

Example 3.1. Let $\mathcal{R}_{2}=\left\{\left(\operatorname{add}_{1}\right),\left(\operatorname{add}_{2}\right),(C),(A)\right\}$ be the TRS for addition of natural numbers and AC-rules for plus given in Example 1.2. Let $\mathcal{S}=\left\{\left(\operatorname{add}_{1}\right),\left(\operatorname{add}_{2}\right)\right\}$ and $\mathcal{P}=$ $\{(C),(A)\}$. Then we have $\mathrm{CP}_{\text {in }}(\mathcal{P}, \mathcal{S})=\emptyset$,

$$
\mathrm{CP}_{\text {out }}(\mathcal{S}, \mathcal{P})=\mathrm{CP}_{\text {out }}(\mathcal{P}, \mathcal{S})^{-1}=\left\{\begin{array}{l}
\langle y,+(y, 0)\rangle \\
\langle\mathrm{s}(+(x, y)),+(y, \mathrm{~s}(x))\rangle
\end{array}\right\}
$$

and

$$
\mathrm{CP}_{i n}(\mathcal{S}, \mathcal{P})=\left\{\begin{array}{l}
\langle+(y, z),+(0,+(y, z))\rangle \\
\langle+(\mathrm{s}(+(x, y)), z),+(\mathrm{s}(x),+(y, z))\rangle
\end{array}\right\}
$$

The parallel extension $\Pi_{\mathcal{R}}$ of the rewrite relation $\rightarrow_{\mathcal{R}}$ of a TRS $\mathcal{R}$ is defined like this: $s \#_{\left\{p_{1}, \ldots, p_{n}\right\}, \mathcal{R}} t$ if $p_{1}, \ldots, p_{n}$ are parallel positions in the term $s$ and there exist rewrite rules $l_{1} \rightarrow r_{1}, \ldots, l_{n} \rightarrow r_{n} \in \mathcal{R}$ and substitution $\theta_{1}, \ldots, \theta_{n}$ such that $s / p_{i}=l_{i} \theta_{i}$ for each $i$ and $t=s\left[r_{1} \theta_{1}, \ldots, r_{n} \theta_{n}\right]_{p_{1}, \ldots, p_{n}}$. If the missing information is clear from the context, $s \mathbb{H}_{\left\{p_{1}, \ldots, p_{n}\right\}, \mathcal{R}} t$ is written as $s \mathbb{H}_{\mathcal{R}} t$ or $s \rightarrow t$. We call $s \mathbb{H}_{\mathcal{R}} t$ a parallel rewrite step. For substitutions $\rho, \rho^{\prime}$, we write $\rho \mathbb{H}_{\mathcal{R}} \rho^{\prime}$ if $\rho(x) \mathbb{H}_{\mathcal{R}} \rho(x)$ for any $x \in \mathcal{V}$. We note that $\mathbb{H}_{\mathcal{R}}$ includes the identity relation, i.e. $t \mathbb{H}_{\mathcal{R}} t$ for any term $t$.

In the rest of this subsection, we present several lemmas that will be used several times in later subsections. The first lemma is used to connect our abstract criterion for ChurchRosser modulo with concrete criteria for confluence. For this, we introduce the notion of reversibility.

Definition 3.2 (reversible relation). A relation $\rightarrow$ is said to be reversible if $\rightarrow \subseteq \stackrel{*}{\leftarrow}$. A TRS $\mathcal{R}$ is reversible if $\rightarrow_{\mathcal{R}}$ is reversible.

Lemma 3.3 (confluence by CRM and reversibility). Let $\mathcal{P}, \mathcal{S}$ be TRSs such that $\mathcal{P}$ is reversible. If $\rightarrow_{\mathcal{S}}$ is Church-Rosser modulo $\stackrel{*}{\leftrightarrow}_{\mathcal{P}}$ then $\mathcal{S} \cup \mathcal{P}$ is confluent.

Proof. Suppose $s \stackrel{*}{\rightarrow} \mathcal{S} \cup \mathcal{P} t_{0}$ and $s \stackrel{*}{\rightarrow} \mathcal{S} \cup \mathcal{P} t_{1}$. Then $t_{0} \stackrel{*}{\rightarrow} \mathcal{S} \cup \mathcal{P} t_{1}$. Since $\rightarrow \mathcal{S}$ is Church-Rosser modulo $\stackrel{*}{\leftrightarrow} \mathcal{P}$, we have $t_{0} \stackrel{*}{\rightarrow} \mathcal{S} u \stackrel{*}{\leftrightarrow} \mathcal{P} v \stackrel{*}{\leftarrow} \mathcal{S} t_{1}$. By the reversibility of $\mathcal{P}$, we have $u \stackrel{*}{\rightarrow} \mathcal{P} v$. Hence $t_{0} \stackrel{*}{\rightarrow} \mathcal{S} \cup \mathcal{P} v \stackrel{*}{\leftarrow} \mathcal{S} \cup \mathcal{P} t_{1}$. 
It is well-known that $\mathcal{S}$ is locally confluent, i.e. $\leftarrow \mathcal{S} \circ \rightarrow_{\mathcal{S}} \subseteq \stackrel{*}{\rightarrow}_{\mathcal{S}} \circ \stackrel{*}{\leftarrow}^{S}$, if $\operatorname{CP}(\mathcal{S}, \mathcal{S}) \subseteq$ $\stackrel{*}{\rightarrow} \mathcal{S} \circ \stackrel{*}{\leftarrow} \mathcal{S}$. The next lemma parametrized this fact by a rewrite relation $\rightarrow$.

Lemma 3.4. Let $\mathcal{S}$ be a TRS and be a rewrite relation. Suppose that $\mathrm{CP}(\mathcal{S}, \mathcal{S}) \subseteq \neg$ and $\stackrel{*}{\rightarrow} \mathcal{S} \circ \stackrel{*}{\leftarrow} \mathcal{S} \subseteq \boldsymbol{\nabla}$. Then $\leftarrow \mathcal{S} \circ \rightarrow \mathcal{S} \subseteq \boldsymbol{\nabla}$.

Proof. Suppose $t_{0} \leftarrow_{p, \mathcal{S}} s \rightarrow_{q, \mathcal{S}} t_{1}$. We distinguish the cases by relative positions of $p$ and $q$. If $p \| q$ then $s=s\left[l \sigma, l^{\prime} \rho\right]_{p, q}$ and $t_{0}=s\left[r \sigma, l^{\prime} \rho\right]_{p, q} \leftarrow_{p} s \rightarrow_{q} s\left[l \sigma, r^{\prime} \rho\right]_{p, q}=t_{1}$ for some rewrite rules $l \rightarrow r, l^{\prime} \rightarrow r^{\prime} \in \mathcal{S}$ and substitutions $\sigma, \rho$. Then we have $t_{0}=s\left[r \sigma, l^{\prime} \rho\right]_{p, q} \rightarrow_{q}$

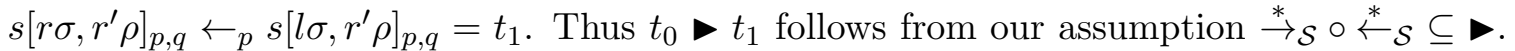
Suppose $q \leq p$. Let $s / q=l \sigma$ and $l \rightarrow r \in \mathcal{S}$. Then either (1) $p / q \in \operatorname{Pos}_{\mathcal{F}}(l)$ or (2) there exists $q_{x} \in \operatorname{Pos} \mathcal{V}(l)$ such that $l / q_{x}=x \in \mathcal{V}$ and $q \cdot q_{x} \leq p$.

(1) Then $t_{0}=s[u \rho]_{q}$ and $t_{1}=s[v \rho]_{q}$ for some $\langle u, v\rangle \in \operatorname{CP}(\mathcal{S}, \mathcal{S})$ and substitution $\rho$. Thus by assumption $u \triangleright v$. Then, since is a rewrite relation, we have $t_{0}=s[u \rho]_{q}>s[v \rho]_{q}=t_{1}$.

(2) Then $t_{1}=s[r \sigma]_{q}$ and $s=s[l \sigma]_{q} \rightarrow_{p, \mathcal{S}} t_{0} \stackrel{*}{\rightarrow} s \quad s\left[l \sigma^{\prime}\right]_{q}$ for some substitution $\sigma^{\prime}$ such that $\sigma(x) \rightarrow_{p /\left(q . q_{x}\right), \mathcal{S}} \sigma^{\prime}(x)$ and $\sigma^{\prime}(y)=\sigma(y)$ for any $y \neq x$. Thus $t_{0} \stackrel{*}{\rightarrow}_{\mathcal{S}} s\left[l \sigma^{\prime}\right]_{q} \rightarrow_{\mathcal{S}}$ $s\left[r \sigma^{\prime}\right]_{q} \stackrel{*}{\leftarrow} \mathcal{S} s[r \sigma]_{q}=t_{1}$. The claim follows from our assumption $\stackrel{*}{\rightarrow} \mathcal{S} \circ \stackrel{*}{\leftarrow} \mathcal{S} \subseteq \triangleright$.

The case of $p<q$ follows similarly to the case of $q \leq p$ using the condition $\operatorname{CP}(\mathcal{S}, \mathcal{S}) \subseteq$

Lemma 3.5. Let $\mathcal{Q}, \mathcal{R}$ be TRSs such that $\mathcal{Q}$ is bidirectional, and let be a rewrite relation.

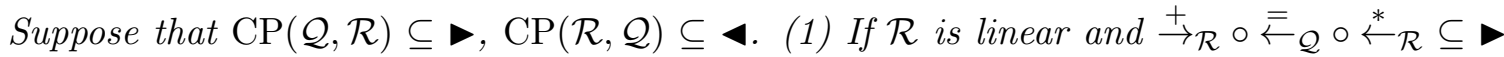
then $\leftarrow_{\mathcal{Q}} \circ \rightarrow_{\mathcal{R}} \subseteq$. (2) If $\mathcal{R}$ is left-linear and $\stackrel{+}{\rightarrow}_{\mathcal{R}} \circ \mathbb{H}_{\mathcal{Q}} \circ \stackrel{*}{\leftarrow}_{\mathcal{R}} \subseteq \triangleright$ then $\leftarrow_{\mathcal{Q}} \circ \rightarrow_{\mathcal{R}} \subseteq \triangleright$.

Proof. Below we present a proof for (1). Any difference to the proof of (2) will be mentioned in the proof. Suppose $t_{0} \leftarrow_{p, \mathcal{Q}} s \rightarrow_{q, \mathcal{R}} t_{1}$. We distinguish the cases by relative positions of $p$ and $q$.

(i) $p \| q$. Then $s=s\left[l \sigma, l^{\prime} \rho\right]_{p, q}$ and $t_{0}=s\left[r \sigma, l^{\prime} \rho\right] \leftarrow_{p, \mathcal{Q}} s \rightarrow_{q, \mathcal{R}} C\left[l \sigma, r^{\prime} \rho\right]_{p, q}=t_{1}$ for some $l \rightarrow r \in \mathcal{Q}, l^{\prime} \rightarrow r^{\prime} \in \mathcal{R}$. Then we have $t_{0}=s\left[r \sigma, l^{\prime} \rho\right]_{p, q} \rightarrow_{q, \mathcal{R}} s\left[r \sigma, r^{\prime} \rho\right]_{p, q} \leftarrow_{p, \mathcal{Q}}$ $s\left[l \sigma, r^{\prime} \rho\right]_{p, q}=t_{1}$. Since $\stackrel{+}{\rightarrow}_{\mathcal{R}} \circ \stackrel{F}{\leftarrow}_{\mathcal{Q}} \circ \stackrel{*}{\leftarrow}_{\mathcal{R}} \subseteq\left(^{+} \stackrel{+}{\rightarrow}_{\mathcal{R}} \circ \mathbb{H}_{\mathcal{Q}} \circ \stackrel{*}{\leftarrow}_{\mathcal{R}} \subseteq \boldsymbol{\nabla}\right.$ in the proof of $\left.(2)\right)$ by our assumption, $t_{0}>t_{1}$.

(ii) $p \leq q$. Let $s / p=l \sigma$ and $l \rightarrow r \in \mathcal{Q}$. Then either (a) $p / q \in \operatorname{Pos}_{\mathcal{F}}(l)$ or (b) there exists $p_{x} \in \operatorname{Pos} \mathcal{V}(l)$ such that $l / p_{x}=x \in \mathcal{V}$ and $p . p_{x} \leq q$.

(a) Then $t_{0}=s[v \rho]_{p}$ and $t_{1}=s[u \rho]_{p}$ for some $\langle u, v\rangle \in \mathrm{CP}(\mathcal{R}, \mathcal{Q})$ and substitution $\rho$. Thus by assumption $v \triangleright u$. Since $\boldsymbol{\triangleright}$ is a rewrite relation, we have $t_{0}=s[v \rho]_{p}$ $s[u \rho]_{p}=t_{1}$.

(b) Then $t_{0}=s[r \sigma]_{p}$ and we have $t_{1} \stackrel{*}{\rightarrow}_{\mathcal{R}} s\left[l \sigma^{\prime}\right]_{p}$ for some substitution $\sigma^{\prime}$ such that $\sigma(x) \rightarrow_{q /\left(p . p_{x}\right), \mathcal{R}} \sigma^{\prime}(x)$ and $\sigma(y)=\sigma^{\prime}(y)$ for any $y \neq x$. Since $\mathcal{Q}$ is bidirectional, we have $\mathcal{V}(l)=\mathcal{V}(r)$. Thus $t_{0}=s[r \sigma]_{p} \stackrel{+}{\rightarrow}_{\mathcal{R}} s\left[r \sigma^{\prime}\right]_{p} \leftarrow_{\mathcal{Q}} s\left[l \sigma^{\prime}\right]_{p} \stackrel{*}{\leftarrow} \mathcal{R} t_{1}$. Since

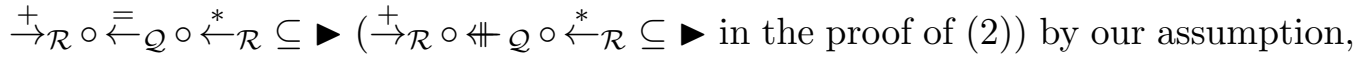
$t_{0} \triangleright t_{1}$.

(iii) $p>q$. Let $s / q=l^{\prime} \rho$ and $l^{\prime} \rightarrow r^{\prime} \in \mathcal{R}$. Then either (a) $p / q \in \operatorname{Pos}_{\mathcal{F}}\left(l^{\prime}\right)$ or (b) there exists $q_{x} \in \operatorname{Pos} \mathcal{V}\left(l^{\prime}\right)$ such that $l^{\prime} / q_{x}=x \in \mathcal{V}$ and $q . q_{x} \leq p$.

(a) Then $t_{0}=s[u \sigma]_{q}$ and $t_{1}=s[v \sigma]_{q}$ for some $\langle u, v\rangle \in \operatorname{CP}(\mathcal{Q}, \mathcal{R})$ and substitution $\sigma$. By assumption $u \triangleright v$. Since $>$ is a rewrite relation, $t_{0}=s[u \sigma]_{q}>s[v \sigma]_{q}=t_{1}$.

(b) Then $t_{1}=s\left[r^{\prime} \rho\right]_{q}$, and by the left-linearity of $\mathcal{R}, t_{0}=s\left[l^{\prime} \rho^{\prime}\right]_{q}$ for some substitution $\rho^{\prime}$ such that $\rho(x) \rightarrow_{p /\left(q . q_{x}\right), \mathcal{Q}} \rho^{\prime}(x)$ and $\rho(y)=\rho^{\prime}(y)$ for any $y \neq x$. Furthermore, 
by the right-linearity of $\mathcal{R}, s\left[r^{\prime} \rho\right]_{q} \stackrel{\bar{J}}{\rightarrow}_{\mathcal{Q}} s\left[r^{\prime} \rho^{\prime}\right]_{q}$. (In the proof of (2), we have $\left.s\left[r^{\prime} \rho\right]_{q} \rightarrow_{\mathcal{Q}} s\left[r^{\prime} \rho^{\prime}\right]_{q}.\right)$ Thus, $t_{0}=s\left[l^{\prime} \rho^{\prime}\right]_{q} \rightarrow_{q, \mathcal{R}} s\left[r^{\prime} \rho^{\prime}\right]_{q} \stackrel{\equiv}{\leftarrow}_{\mathcal{Q}} s\left[r^{\prime} \rho\right]_{q}=t_{1}\left(t_{0}=\right.$

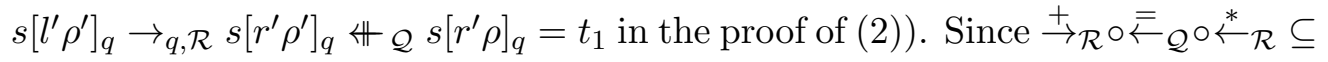

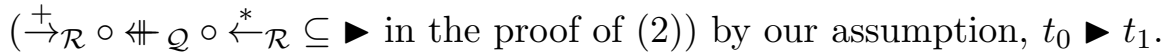

3.2. Confluence criterion for linear TRSs. In this subsection, we give a confluence criterion for TRSs that can be partitioned into linear terminating TRS $\mathcal{S}$ and reversible TRS $\mathcal{P}$. We then discuss the possibility of relaxing linearity condition to left-linearity.

The next two lemmas are corollaries of Lemmas 3.4 and 3.5 , respectively.

Lemma 3.6. Let $\mathcal{P}, \mathcal{S}, \mathcal{P}^{\prime}$ be TRSs such that $\mathcal{P}$ is bidirectional. Suppose $\operatorname{CP}(\mathcal{S}, \mathcal{S}) \subseteq$

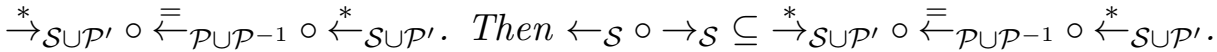

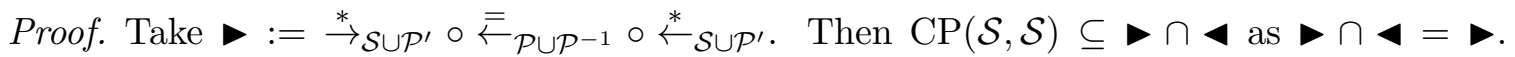
Furthermore, we have $\stackrel{*}{\rightarrow} \mathcal{S} \circ \stackrel{*}{\leftarrow} \mathcal{S} \subseteq \boldsymbol{~ . ~ H e n c e ~ t h e ~ c l a i m ~ f o l l o w s ~ f r o m ~ L e m m a ~} 3.4$.

Lemma 3.7. Let $\mathcal{P}, \mathcal{S}, \mathcal{P}^{\prime}$ be TRSs such that $\mathcal{P}$ is bidirectional and $\mathcal{S}$ is linear. Let $\mathbf{}=$

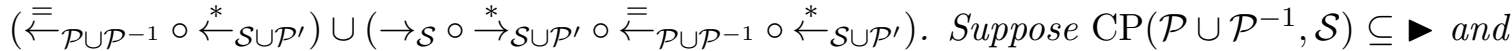
$\mathrm{CP}\left(\mathcal{S}, \mathcal{P} \cup \mathcal{P}^{-1}\right) \subseteq$ ४. Then $\leftarrow \mathcal{P} \cup \mathcal{P}^{-1} \circ \rightarrow \mathcal{S} \subseteq$.

Proof. Take $\mathcal{Q}:=\mathcal{P} \cup \mathcal{P}^{-1}$, which is a bidirectional TRS by bidirectionality of $\mathcal{P}$, and $\mathcal{R}:=\mathcal{S}$ in Lemma 3.5. Then by the condition, $\operatorname{CP}\left(\mathcal{P} \cup \mathcal{P}^{-1}, \mathcal{S}\right) \subseteq \boldsymbol{\vee}$ and $\operatorname{CP}\left(\mathcal{S}, \mathcal{P} \cup \mathcal{P}^{-1}\right) \subseteq \varangle$. Furthermore, $\stackrel{+}{\rightarrow}_{\mathcal{R}} \circ \stackrel{\equiv}{\leftarrow}_{\mathcal{Q}} \circ \stackrel{*}{\leftarrow}_{\mathcal{R}}=\stackrel{+}{\rightarrow}_{\mathcal{S}} \circ \stackrel{\equiv}{\leftarrow}_{\mathcal{P} \cup \mathcal{P}^{-1}} \circ \stackrel{*}{\leftarrow}_{\mathcal{S}} \subseteq \triangleright$. Hence the claim follows from Lemma $3.5(1)$.

We arrive at our first criterion for confluence.

Theorem 3.8 (confluence criterion for linear $\mathcal{S}$ ). Let $\mathcal{P}, \mathcal{S}, \mathcal{P}^{\prime}$ be TRSs such that $\mathcal{S}$ is linear, $\mathcal{P}$ is reversible, $\mathcal{P}^{\prime} \subseteq \mathcal{P} \cup \mathcal{P}^{-1}$ and $\mathcal{S}$ is terminating relative to $\mathcal{P}^{\prime}$. Suppose (i) $\operatorname{CP}(\mathcal{S}, \mathcal{S}) \subseteq$

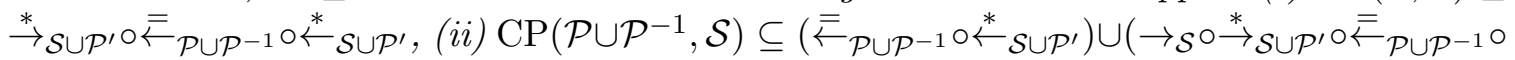

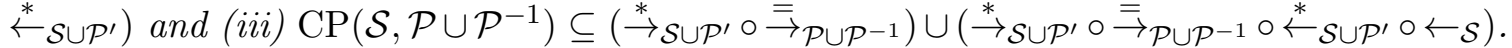
Then $\mathcal{S} \cup \mathcal{P}$ is confluent.

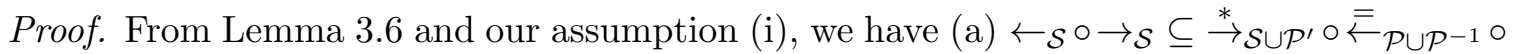

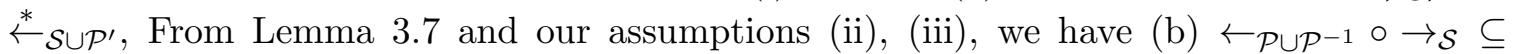
$\left({\stackrel{F}{\leftarrow} \mathcal{P} \cup \mathcal{P}^{-1}} \circ \stackrel{*}{\leftarrow}_{\mathcal{S} \cup \mathcal{P}^{\prime}}\right) \cup\left(\rightarrow \mathcal{S} \circ \stackrel{*}{\rightarrow}_{\mathcal{S} \cup \mathcal{P}^{\prime}} \circ \stackrel{\Sigma}{\leftarrow}_{\mathcal{P} \cup \mathcal{P}^{-1}} \circ \stackrel{*}{\leftarrow}_{\mathcal{S} \cup \mathcal{P}^{\prime}}\right)$. Note that, by the definition of rewrite rules, reversible TRSs are bidirectional. Take $\mapsto:=\leftrightarrow_{\mathcal{P}}=\leftarrow \mathcal{P} \cup \mathcal{P}^{-1}, \leadsto:=\rightarrow \mathcal{P}^{\prime}$ and $\rightarrow:=\rightarrow \mathcal{S}$. Then since $\mathcal{S}$ is terminating relative to $\mathcal{P}^{\prime}$, the relation $\rightarrow 0 \stackrel{*}{\sim}$ is well-founded. Thus one can apply Theorem 2.2 so as to prove $\rightarrow \mathcal{S}$ is Church-Rosser modulo $\stackrel{*}{\leftrightarrow} \mathcal{P}$. Since $\mathcal{P}$ is reversible, $\rightarrow \mathcal{S} \cup \mathcal{P}$ is confluent by Lemma 3.3 .

Remark 3.9. Conditions like (i)-(iii) are referred to as critical pair conditions in the sequel.

By taking $\mathcal{P}^{\prime}=\emptyset$ in Theorem 3.8 , we obtain the next corollary.

Corollary 3.10 (Theorem 1 of [10]). Let $\mathcal{P}, \mathcal{S}$ be TRSs such that $\mathcal{S}$ is linear, $\mathcal{P}$ is reversible and $\mathcal{S}$ is terminating. Suppose (i) $\operatorname{CP}(\mathcal{S}, \mathcal{S}) \subseteq \stackrel{*}{\rightarrow}_{\mathcal{S}} \circ \stackrel{F_{\leftarrow}^{\leftarrow}}{\leftarrow} \cup \mathcal{P}^{-1} \circ \stackrel{*}{\leftarrow}_{\mathcal{S}}$, (ii) $\operatorname{CP}\left(\mathcal{P} \cup \mathcal{P}^{-1}, \mathcal{S}\right) \subseteq$

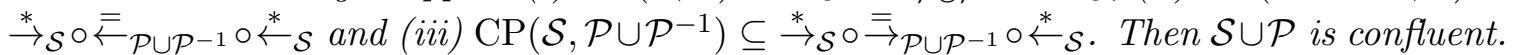


To generalize Theorem 3.8 for (possibly not right-linear) left-linear $\mathcal{S}$, we have to use Lemma 3.5 (2) instead of Lemma 3.5 (1). For this, we needs condition $\stackrel{+}{\rightarrow}_{\mathcal{R}} \circ \mathbb{H}_{\mathcal{Q}} \circ \stackrel{*}{\leftarrow}_{\mathcal{R}} \subseteq$ -. However, this fact reduces the application of Theorem 2.2 to just an application of Corollary 2.5 (and hence that of Corollary 2.6), because the $=$-step in the condition does not contribute and hence we have to take $\leadsto:=\mapsto$. In the rest of this subsection, we sketch how a proof analogous to the linear case can be applied to obtain a confluence criterion based on the Corollary 2.6 .

Lemma 3.11. Let $\mathcal{P}, \mathcal{S}$ be TRSs such that $\mathcal{P}$ is bidirectional. Suppose $\operatorname{CP}(\mathcal{S}, \mathcal{S}) \subseteq{ }^{*} \rightarrow \mathcal{S} \circ$

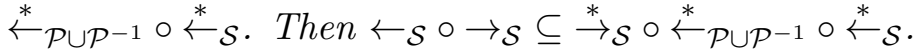

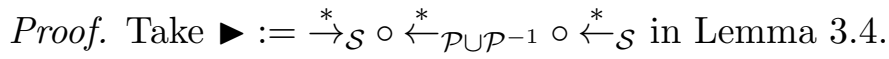

Lemma 3.12. Let $\mathcal{P}, \mathcal{S}$ be TRSs such that $\mathcal{P}$ is bidirectional and $\mathcal{S}$ is left-linear. Suppose (i) $\mathrm{CP}\left(\mathcal{P} \cup \mathcal{P}^{-1}, \mathcal{S}\right) \subseteq \stackrel{+}{\rightarrow} \mathcal{S} \circ \stackrel{*}{\leftarrow} \mathcal{P} \cup \mathcal{P}^{-1} \circ \stackrel{*}{\leftarrow} \mathcal{S}$ and (ii) $\mathrm{CP}\left(\mathcal{S}, \mathcal{P} \cup \mathcal{P}^{-1}\right) \subseteq \stackrel{*}{\rightarrow} \mathcal{S} \circ \stackrel{*}{\rightarrow} \mathcal{P} \cup \mathcal{P}^{-1} \circ \stackrel{+}{\leftarrow} \mathcal{S}$. Then $\leftarrow \mathcal{P} \cup \mathcal{P}^{-1} \circ \rightarrow \mathcal{S} \subseteq \stackrel{+}{\rightarrow}_{\mathcal{S}} \circ \stackrel{*}{\leftarrow} \mathcal{P} \cup \mathcal{P}^{-1} \circ \stackrel{*}{\leftarrow} \mathcal{S}$.

Proof. Take $:=\stackrel{+}{\rightarrow}_{\mathcal{S}} \circ \stackrel{*}{\leftarrow}_{\mathcal{P} \cup \mathcal{P}^{-1}} \circ \stackrel{*}{\leftarrow} \mathcal{S}$ in Lemma 3.5 (2).

Proposition 3.13 (Theorem 3.3 of [8]). Let $\mathcal{P}, \mathcal{S}$ be TRSs such that $\mathcal{S}$ is left-linear, $\mathcal{P}$ is reversible and $\mathcal{S}$ is terminating relative to $\mathcal{P}$. Suppose (i) $\mathrm{CP}(\mathcal{S}, \mathcal{S}) \subseteq \stackrel{*}{\rightarrow}_{\mathcal{S}} \circ \stackrel{*}{\leftarrow} \mathcal{P} \cup \mathcal{P}^{-1} \circ \stackrel{*}{\leftarrow} \mathcal{S}$

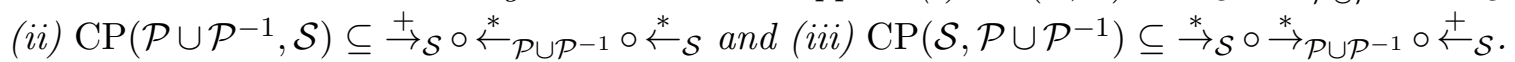
Then $\mathcal{S} \cup \mathcal{P}$ is confluent.

Proof. Since $\mathcal{P}$ is reversible, $\mathcal{S}$ is terminating modulo $\mathcal{P} \cup \mathcal{P}^{-1}$. By Lemmas 3.11 and 3.12 and Corollary 2.6. $\rightarrow_{\mathcal{S}}$ is Church-Rosser modulo $\stackrel{*}{\rightarrow} \mathcal{P}$. Since $\mathcal{P}$ is reversible, $\rightarrow_{\mathcal{S} \cup \mathcal{P}}$ is confluent by Lemma 3.3 .

Remark 3.14. It is straightforward to modify Lemmas 3.11 and 3.12 and use either Corollary 2.5 (or Corollary 2.8) to replace conditions (i)-(iii) of Proposition 3.13 with (i)

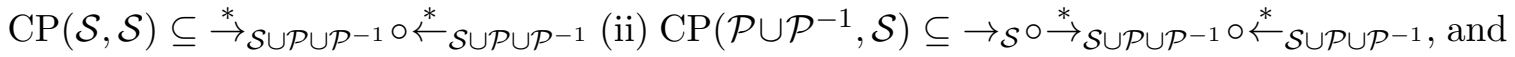

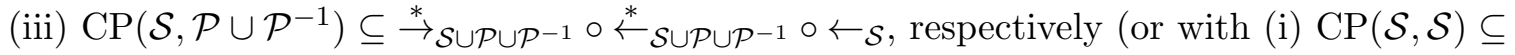
$\stackrel{*}{\rightarrow} \mathcal{S} \circ \stackrel{*}{\leftarrow} \mathcal{P} \cup \mathcal{P}^{-1} \circ \stackrel{*}{\leftarrow} \mathcal{S}$, (ii) $\mathrm{CP}\left(\mathcal{P} \cup \mathcal{P}^{-1}, \mathcal{S}\right) \subseteq \stackrel{*}{\rightarrow} \mathcal{S} \circ \stackrel{*}{\leftarrow} \mathcal{P} \cup \mathcal{P}^{-1} \circ \stackrel{*}{\leftarrow} \mathcal{S}$, and (iii) $\operatorname{CP}\left(\mathcal{S}, \mathcal{P} \cup \mathcal{P}^{-1}\right) \subseteq$ $\stackrel{*}{\rightarrow}_{\mathcal{S}} \circ \stackrel{*}{\rightarrow}_{\mathcal{P} \cup \mathcal{P}^{-1}} \circ \stackrel{*}{\leftarrow} \mathcal{S}$, respectively). Similar to the abstract case, such replacements do not strengthen or weaken the applicability of the proposition (c.f. Remark 2.7).

3.3. Confluence criterion based on parallel rewrite steps. As discussed in the previous subsection, if we put $\mapsto:=\leftrightarrow_{\mathcal{P}}=\leftarrow_{\mathcal{P} \cup \mathcal{P}^{-1}}$, the application of our abstract criterion (Theorem 2.2) to the left-linear case reduces to the application of Corollary 2.6. In this subsection, we relax the linear condition of the $\mathcal{S}$-part to left-linear by considering

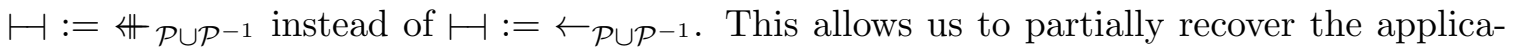
bility of Theorem 2.2 .

The next lemma is analogous to Lemma 3.6, which is obtained from Lemma 3.4 again.

Lemma 3.15. Let $\mathcal{P}, \mathcal{S}, \mathcal{P}^{\prime}$ be TRSs such that $\mathcal{P}$ is bidirectional. Suppose that $\operatorname{CP}(\mathcal{S}, \mathcal{S}) \subseteq$

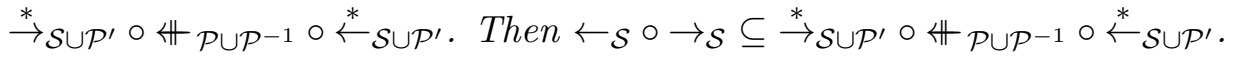

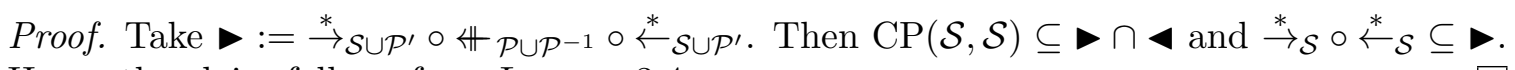
Hence the claim follows from Lemma 3.4 . 
To present an analogy of Lemma 3.7, we first present the parametrized version of the lemma in the same spirit as Lemma 3.5 .

Lemma 3.16. Let $\mathcal{Q}, \mathcal{R}$ be TRSs such that $\mathcal{Q}$ is bidirectional and $\mathcal{R}$ is left-linear. Let be a rewrite relation such that $s_{i} \triangleright t_{i}$ and $s_{j} \leftarrow_{\mathcal{Q}} t_{j}$ for any $j \in\{1, \ldots, n\} \backslash\{i\}$ implies $C\left[s_{1}, \ldots, s_{n}\right] \triangleright C\left[t_{1}, \ldots, t_{n}\right]$. Suppose (i) $\mathrm{CP}_{\text {in }}(\mathcal{Q}, \mathcal{R})=\emptyset$ and (ii) $\mathrm{CP}(\mathcal{R}, \mathcal{Q}) \subseteq \triangleleft$. If $\stackrel{+}{\rightarrow}_{\mathcal{R}} \circ \mathbb{H}_{\mathcal{Q}} \circ \stackrel{*}{\leftarrow}_{\mathcal{R}} \subseteq$ then $\mathbb{H}_{\mathcal{Q}} \circ \rightarrow_{\mathcal{R}} \subseteq \boldsymbol{\nabla}$.

Proof. Suppose $t_{0} \mathbb{H}_{U, \mathcal{Q}} s \rightarrow_{q, \mathcal{R}} t_{1}$. Let $U=\left\{p_{1}, \ldots, p_{n}\right\}$ where $p_{1}, \ldots, p_{n}$ are positions from left to right, $s / p_{i}=l_{i} \sigma_{i}$ for $l_{i} \rightarrow r_{i} \in \mathcal{Q}$ and substitutions $\sigma_{i}(1 \leq i \leq n)$ and $s / q=l^{\prime} \rho$ for $l^{\prime} \rightarrow r^{\prime} \in \mathcal{R}$ and a substitution $\rho$. We distinguish two cases: (1) the case that $\exists p \in U . p \leq q$ and (2) the case that $\forall p \in U . p \not \leq q$.

(1) Suppose $p_{i} \in U$ and $p_{i} \leq q$. Then either (a) $q / p_{i} \in \operatorname{Pos}_{\mathcal{F}}\left(l_{i}\right)$ or (b) there exists $p_{x} \in \operatorname{Pos} \mathcal{V}\left(l_{i}\right)$ such that $l_{i} / p_{x}=x \in \mathcal{V}$ and $p_{i} \cdot p_{x} \leq q$.

(a) Then $t_{0} / p_{i}=v \rho$ and $t_{1} / p_{i}=u \rho$ for some $\langle u, v\rangle \in \operatorname{CP}(\mathcal{R}, \mathcal{Q})$ and substitution $\rho$. Then, from our assumption (ii), we have $v \triangleright u$. Since $>$ is a rewrite relation, $t_{0} / p_{i}=v \rho \triangleright u \rho=t_{1} / p_{i}$. By the assumption on $\triangleright$, since for any $j \neq i$, $s / p_{j}=r_{j} \sigma_{j} \leftarrow_{\mathcal{Q}} l_{j} \sigma_{j}=t_{1} / p_{j}$, we have $t_{0}=s\left[r_{1} \sigma_{1}, \ldots, t_{0} / p_{i}, \ldots, r_{n} \sigma_{n}\right]_{p_{1}, \ldots, p_{i}, \ldots, p_{n}}$ $s\left[l_{1} \sigma_{1}, \ldots, t_{1} / p_{i}, \ldots, l_{n} \sigma_{n}\right]_{p_{1}, \ldots, p_{i}, \ldots, p_{n}}=t_{1}$.

(b) Then we have $t_{0} / p_{i}=r_{i} \sigma_{i}$ and $t_{1} / p_{i} \stackrel{*}{\rightarrow}_{\mathcal{R}} l_{i} \sigma_{i}^{\prime}$ for some substitution $\sigma_{i}^{\prime}$ such that $\sigma_{i}(x) \rightarrow_{q /\left(p_{i} . p_{x}\right), \mathcal{R}} \sigma_{i}^{\prime}(x)$ and $\sigma_{i}^{\prime}(y)=\sigma_{i}(y)$ for any $y \neq x$. Since $\mathcal{Q}$ is bidirectional, we have $\mathcal{V}\left(l_{i}\right)=\mathcal{V}\left(r_{i}\right)$. Thus we have

$$
\begin{array}{rll}
t_{0} & = & C\left[r_{1} \sigma_{1}, \ldots, r_{i} \sigma_{i}, \ldots, r_{n} \sigma_{n}\right]_{p_{1}, \ldots, p_{i}, \ldots, p_{n}} \\
& \stackrel{+}{\mathcal{R}}_{\mathcal{R}} & C\left[r_{1} \sigma_{1}, \ldots, r_{i} \sigma_{i}^{\prime}, \ldots, r_{n} \sigma_{n}\right]_{p_{1}, \ldots, p_{i}, \ldots, p_{n}} \\
& \mathbb{H}_{\mathcal{Q}} & C\left[l_{1} \sigma_{1}, \ldots, l_{i} \sigma_{i}^{\prime}, \ldots, l_{n} \sigma_{n}\right]_{p_{1}, \ldots, p_{i}, \ldots, p_{n}} \\
& { }^{*} \mathcal{R} & C\left[l_{1} \sigma_{1}, \ldots, t_{1} / p_{i}, \ldots, l_{n} \sigma_{n}\right]_{p_{1}, \ldots, p_{i}, \ldots, p_{n}}=t_{1} .
\end{array}
$$

From our assumption that $\stackrel{+}{\rightarrow}_{\mathcal{R}} \circ \mathbb{H}_{\mathcal{Q}} \circ \stackrel{*}{\leftarrow}_{\mathcal{R}} \subseteq \triangleright$ it follows $t_{0} \triangleright t_{1}$.

(2) Suppose $\forall p \in U . p \not \leq q$. Let $U^{\prime}=\left\{p_{i} \in U \mid q<p_{i}\right\}=\left\{p_{l}, \ldots, p_{k}\right\}, q_{i}=p_{i} / q$ for $l \leq i \leq k$, and thus $l^{\prime} \rho=l^{\prime} \rho\left[l_{l} \sigma_{l}, \ldots, l_{k} \sigma_{k}\right]_{q_{l}, \ldots, q_{k}}$. By our assumption (i), for each $p_{i} \in \overline{U^{\prime}}$ there exists $q_{x} \in \operatorname{Pos} \mathcal{V}\left(l^{\prime}\right)$ such that $l^{\prime} / q_{x}=x \in \mathcal{V}$ and $q . q_{x} \leq p_{i}$. Thus, $s / q=l^{\prime} \rho=l^{\prime} \rho\left[l_{l} \sigma_{l}, \ldots, l_{k} \sigma_{k}\right]_{q_{l}, \ldots, q_{k}} \rightarrow \mathcal{R} r^{\prime} \rho=r^{\prime} \rho\left[l_{j_{1}} \sigma_{j_{1}}, \ldots, l_{j_{m}} \sigma_{j_{m}}\right]_{o_{1}, \ldots, o_{m}}=t_{1} / q$ for some positions $o_{1}, \cdots, o_{m}$ and $j_{1}, \ldots, j_{m} \in\{l, \ldots, k\}$. Furthermore, by the left-linearity of $\mathcal{R}$, we have $l^{\prime} \rho\left[r_{l} \sigma_{l}, \ldots, r_{k} \sigma_{k}\right]_{q_{l}, \ldots, q_{k}} \rightarrow_{\mathcal{R}} r^{\prime} \rho\left[r_{j_{1}} \sigma_{j_{1}}, \ldots, r_{j_{m}} \sigma_{j_{m}}\right]_{o_{1}, \ldots, o_{m}}$. Thus,

$$
\begin{aligned}
& t_{0}=s\left[r_{1} \sigma_{1}, \ldots, l^{\prime} \rho\left[r_{l} \sigma_{l}, \ldots, r_{k} \sigma_{k}\right]_{q_{l}, \ldots, q_{k}}, \ldots, r_{n} \sigma_{n}\right]_{p_{1}, \ldots, q, \ldots, p_{n}} \\
& \rightarrow_{\mathcal{R}} \quad s\left[r_{1} \sigma_{1}, \ldots, r^{\prime} \rho\left[r_{j_{1}} \sigma_{j_{1}}, \ldots, r_{j_{m}} \sigma_{j_{m}}\right]_{o_{1}, \ldots, o_{m}}, \ldots, r_{n} \sigma_{n}\right]_{p_{1}, \ldots, q, \ldots, p_{n}} \\
& \mathbb{H}_{\mathcal{Q}} s\left[l_{1} \sigma_{1}, \ldots, r^{\prime} \rho\left[l_{j_{1}} \sigma_{j_{1}}, \ldots, l_{j_{m}} \sigma_{j_{m}}\right]_{o_{1}, \ldots, o_{m}}, \ldots, l_{n} \sigma_{n}\right]_{p_{1}, \ldots, q, \ldots, p_{n}}=t_{1} \text {. }
\end{aligned}
$$

From our assumption that $\stackrel{+}{\rightarrow}_{\mathcal{R}} \circ \# \mathbb{Q}_{\mathcal{O}} \circ \stackrel{*}{\leftarrow}_{\mathcal{R}} \subseteq$ follows $t_{0} \triangleright t_{1}$.

The next lemma is an analogy of Lemma 3.7 based on parallel steps.

Lemma 3.17. Let $\mathcal{P}, \mathcal{S}, \mathcal{P}^{\prime}$ be TRSs such that $\mathcal{P}$ is bidirectional and $\mathcal{S}$ is left-linear. Let

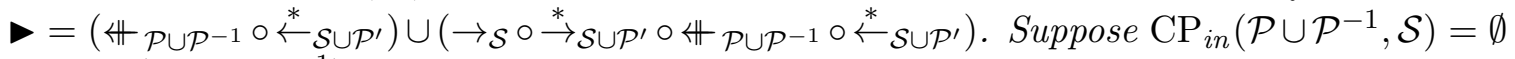
and $\mathrm{CP}\left(\mathcal{S}, \mathcal{P} \cup \mathcal{P}^{-1}\right) \subseteq \varangle$. Then $\mathbb{H}_{\mathcal{P} \cup \mathcal{P}^{-1}} \circ \rightarrow_{\mathcal{S}} \subseteq \triangleright$.

Proof. Take $\mathcal{Q}:=\mathcal{P} \cup \mathcal{P}^{-1}$, which is a bidirectional TRS by bidirectionality of $\mathcal{P}$, and $\mathcal{R}:=\mathcal{S}$ in Lemma 3.16. Then by the condition, $\mathrm{CP}_{i n}(\mathcal{Q}, \mathcal{R})=\emptyset$ and $\operatorname{CP}(\mathcal{R}, \mathcal{Q}) \subseteq \varangle$. Furthermore, 
$\stackrel{+}{\rightarrow}_{\mathcal{R}} \circ \mathbb{H}_{\mathcal{Q}} \circ \stackrel{*}{\leftarrow} \mathcal{R} \subseteq \rightarrow_{\mathcal{S}} \circ \stackrel{*}{\rightarrow}_{\mathcal{S} \cup \mathcal{P}^{\prime}} \circ \mathbb{H}_{\mathcal{P} \cup \mathcal{P}^{-1}} \circ \stackrel{*}{\leftarrow} \mathcal{S} \cup \mathcal{P}^{\prime} \subseteq \triangleright$ and $s_{i} \bullet t_{i}$ and $s_{j} \leftarrow_{\mathcal{Q}} t_{j}$ for any $j \in\{1, \ldots, n\} \backslash\{i\}$ implies $C\left[s_{1}, \ldots, s_{n}\right] \triangleright C\left[t_{1}, \ldots, t_{n}\right]$. Hence the claim follows from Lemma 3.16 .

We arrive at our second criterion for confluence.

Theorem 3.18 (confluence criterion based on parallel rewrite steps). Let $\mathcal{P}, \mathcal{S}, \mathcal{P}^{\prime}$ be TRSs such that $\mathcal{S}$ is left-linear, $\mathcal{P}$ is reversible, $\mathcal{P}^{\prime} \subseteq \mathcal{P} \cup \mathcal{P}^{-1}$, and $\mathcal{S}$ is terminating relative to

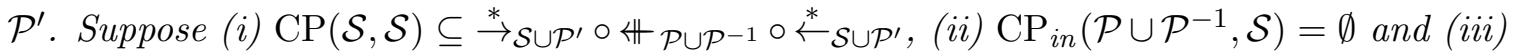

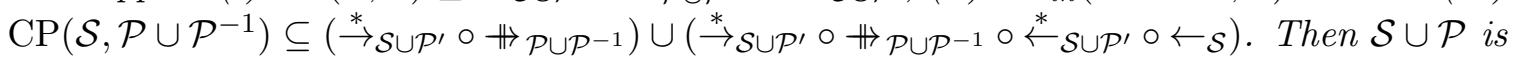
confluent.

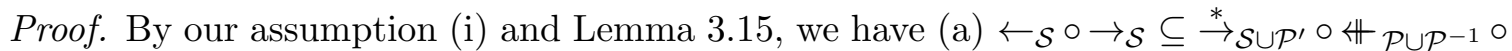

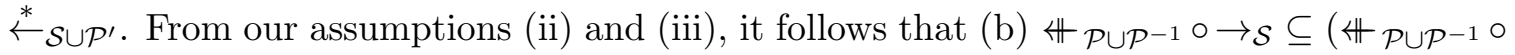
$\left.\stackrel{*}{\leftarrow} \mathcal{S} \cup \mathcal{P}^{\prime}\right) \cup\left(\rightarrow \mathcal{S} \circ \stackrel{*}{\rightarrow} \mathcal{S} \cup \mathcal{P}^{\prime} \circ \mathbb{H} \mathcal{P} \cup \mathcal{P}^{-1} \circ \stackrel{*}{\leftarrow} \mathcal{S} \cup \mathcal{P}^{\prime}\right)$ by Lemma 3.17. Take $\mapsto:=\mathbb{P} \cup \mathcal{P}^{-1}, \rightarrow:=\rightarrow \mathcal{S}$ and $\leadsto:=\rightarrow \mathcal{P}^{\prime}$. Then, by the termination of $\mathcal{S}$ relative to $\mathcal{P}^{\prime}, \rightarrow \circ \stackrel{*}{\sim}$ is well-founded. Thus one can apply Theorem 2.2 so as to prove $\rightarrow_{\mathcal{S}}$ is Church-Rosser modulo $\stackrel{*}{*}_{\mathcal{P} \cup \mathcal{P}^{-1}}$. Since $\stackrel{*}{\mathbb{H}_{\mathcal{P}} \cup \mathcal{P}^{-1}}=\stackrel{*}{\leftrightarrow}_{\mathcal{P}}$, it follows that $\rightarrow_{\mathcal{S}}$ is Church-Rosser modulo $\stackrel{*}{\leftrightarrow} \mathcal{P}$. Hence, since $\rightarrow_{\mathcal{P}}$ is reversible, $\rightarrow \mathcal{S} \cup \mathcal{P}$ is confluent by Lemma 3.3 .

Comparing to our first criterion for confluence (Theorem 3.8), we impose the condition $\mathrm{CP}_{\text {in }}\left(\mathcal{P} \cup \mathcal{P}^{-1}, \mathcal{S}\right)=\emptyset$ while relaxing the linearity condition of $\mathcal{S}$ to left-linearity. Hence Theorems 3.8 and 3.18 are incomparable.

By taking $\mathcal{P}^{\prime}=\emptyset$ in Theorem 3.18 , we obtain the next corollary.

Corollary 3.19. Let $\mathcal{P}, \mathcal{S}$ be TRSs such that $\mathcal{S}$ is left-linear, $\mathcal{P}$ is reversible, and $\mathcal{S}$ is terminating. Suppose (i) $\mathrm{CP}(\mathcal{S}, \mathcal{S}) \subseteq \stackrel{*}{\rightarrow} \mathcal{S} \circ \mathbb{H}_{\mathcal{P} \cup \mathcal{P}^{-1}} \circ \stackrel{*}{\leftarrow}^{S}$, (ii) $\mathrm{CP}_{\text {in }}\left(\mathcal{P} \cup \mathcal{P}^{-1}, \mathcal{S}\right)=\emptyset$ and (iii) $\mathrm{CP}\left(\mathcal{S}, \mathcal{P} \cup \mathcal{P}^{-1}\right) \subseteq \stackrel{*}{\rightarrow}_{\mathcal{S}} \circ \mathbb{H}_{\mathcal{P} \cup \mathcal{P}^{-1}} \circ \stackrel{*}{\leftarrow} \mathcal{S}$. Then $\mathcal{S} \cup \mathcal{P}$ is confluent.

3.4. Confluence criterion based on parallel critical pairs. In this subsection, we relax the condition (ii) $\mathrm{CP}_{i n}\left(\mathcal{P} \cup \mathcal{P}^{-1}, \mathcal{S}\right)=\emptyset$ of the previous theorem using the notion of parallel critical pairs [7, 20].

Definition 3.20 (parallel critical pairs [7, 20]). Let $s_{1}, \ldots, s_{n}, t(n \geq 1)$ be terms whose variables are disjoint. The terms $s_{1}, \ldots, s_{n}$ parallel-overlap on $t$ (at parallel positions $\left.p_{1}, \ldots, p_{n}\right)$ if $t / p_{i} \notin \mathcal{V}$ for any $1 \leq i \leq n$ and $\left\{s_{1} \approx t / p_{1}, \ldots, s_{n} \approx t / p_{n}\right\}$ is unifiable. Let $l_{1} \rightarrow r_{1}, \ldots, l_{n} \rightarrow r_{n}$ and $l^{\prime} \rightarrow r^{\prime}$ be rewrite rules. W.l.o.g. let their variables be mutually disjoint. Suppose that $l_{1}, \ldots, l_{n}$ parallel-overlap on $l^{\prime}$ at parallel positions $p_{1}, \ldots, p_{n}$ and $\sigma$ is the mgu of $\left\{l_{1} \approx l^{\prime} / p_{1}, \ldots, l_{n} \approx l^{\prime} / p_{n}\right\}$. Then the term $l^{\prime}\left[l_{1}, \ldots, l_{n}\right]_{p_{1}, \ldots, p_{n}} \sigma$ yields a parallel critical pair $\left\langle l^{\prime}\left[r_{1}, \ldots, r_{n}\right]_{p_{1}, \ldots, p_{n}} \sigma, r^{\prime} \sigma\right\rangle$ obtained by the parallel-overlap of $l_{1} \rightarrow r_{1}, \ldots, l_{n} \rightarrow r_{n}$ on $l^{\prime} \rightarrow r^{\prime}$ at positions $p_{1}, \ldots, p_{n}$. In the case of self-overlap (i.e. when $n=1$ and $l_{1} \rightarrow r_{1}$ and $l^{\prime} \rightarrow r^{\prime}$ are identical modulo renaming), we do not consider the case $p_{1}=\epsilon$. We write $\left\langle l^{\prime}\left[r_{1}, \ldots, r_{n}\right]_{p_{1}, \ldots, p_{n}} \sigma, r^{\prime} \sigma\right\rangle_{X}$ if $X=\mathcal{V}_{\left\{p_{1}, \ldots, p_{n}\right\}}\left(l^{\prime} \sigma\right)$. We call the parallel critical pair outer if $n=1$ and $p_{1}=\epsilon$, and inner if $p_{i}>\epsilon$ for all $i$. The set of outer (inner) parallel critical pairs obtained by the parallel-overlaps of rewrite rules from $\mathcal{R}$ on a rewrite rule from $\mathcal{Q}$ is denoted by $\mathrm{PCP}_{\text {out }}(\mathcal{R}, \mathcal{Q})\left(\mathrm{PCP}_{\text {in }}(\mathcal{R}, \mathcal{Q})\right.$, respectively). (Note, however, that $\operatorname{PCP}_{\text {out }}(\mathcal{R}, \mathcal{Q})=\mathrm{CP}_{\text {out }}(\mathcal{R}, \mathcal{Q})$.) We put $\operatorname{PCP}(\mathcal{R}, \mathcal{Q})=\operatorname{PCP}_{\text {out }}(\mathcal{R}, \mathcal{Q}) \cup \mathrm{PCP}_{\text {in }}(\mathcal{R}, \mathcal{Q})$. 
Example 3.21 (parallel critical pairs). Let $\mathcal{R}=\{\mathrm{f}(\mathrm{g}(x), \mathrm{g}(y)) \rightarrow \mathrm{h}(\mathrm{g}(x))\}$ and $\mathcal{Q}=$ $\{\mathrm{g}(x) \rightarrow \mathrm{h}(x)\}$. Then we have $\operatorname{PCP}(\mathcal{R}, \mathcal{Q})=\operatorname{PCP}_{\text {out }}(\mathcal{Q}, \mathcal{R})=\emptyset$ and $\operatorname{PCP}_{\text {in }}(\mathcal{Q}, \mathcal{R})=$ $\left\{\langle\mathrm{f}(\mathrm{h}(x), \mathrm{h}(y)), \mathrm{h}(\mathrm{g}(x))\rangle_{\{x, y\}},\langle\mathrm{f}(\mathrm{g}(x), \mathrm{h}(y)), \mathrm{h}(\mathrm{g}(x))\rangle_{\{y\}},\langle\mathrm{f}(\mathrm{h}(x), \mathrm{g}(y)), \mathrm{h}(\mathrm{g}(x))\rangle_{\{x\}}\right\}$.

We first present a property of parallel rewrite steps that will be used to prove a key lemma below.

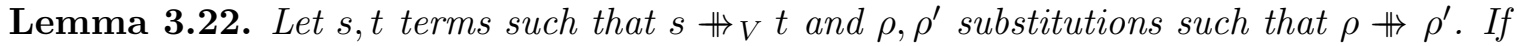
$\operatorname{dom}(\rho) \cap \mathcal{V}_{V}(t)=\emptyset$ then $s \rho \pitchfork t \rho^{\prime}$.

Proof. We have $s \rho \mathbb{H}_{V} t \rho \mathbb{H}_{W} t \rho^{\prime}$ for some $W$ such that for any $q \in W$ there exists $q^{\prime} \in \operatorname{Pos}_{\operatorname{dom}(\rho)}(t)$ such that $q^{\prime} \leq q$. Since $\operatorname{dom}(\rho) \cap \mathcal{V}_{V}(t)=\emptyset, p \| q^{\prime}$ holds for any $p \in V$ and $q^{\prime} \in \operatorname{Pos}_{\operatorname{dom}(\rho)}(t)$. Thus $p \| q$ for any $p \in V$ and $q \in W$. Hence $s \rho \boxplus t \rho^{\prime}$.

The following lemma is a key lemma which shows the preservation of parallel rewrite steps via substitutions. For this lemma, a variable condition on parallel critical pairs and parallel rewrite steps is essential.

Lemma 3.23. Let $\mathcal{S}, \mathcal{P}$ be TRSs, $\langle u, v\rangle_{X} \in \mathrm{PCP}_{\text {in }}(\mathcal{P}, \mathcal{S})$ and $\rho, \rho^{\prime}$ substitutions such that $\rho \# \mathcal{P} \rho^{\prime}$ and $\operatorname{dom}(\rho) \cap X=\emptyset$. If $u \stackrel{*}{\rightarrow} u^{\prime} \mathbb{H}_{V, \mathcal{P}} v^{\prime} \stackrel{*}{\leftarrow} v$ and $\mathcal{V}_{V}\left(u^{\prime}\right) \subseteq X$ then $u \rho^{\prime} \stackrel{*}{\rightarrow} u^{\prime} \rho^{\prime} \mathbb{H}_{\mathcal{P}}$ $v^{\prime} \rho \stackrel{*}{\leftarrow} v \rho$, where $u \rho^{\prime} \stackrel{*}{\rightarrow} u^{\prime} \rho^{\prime}$ and $v^{\prime} \rho \stackrel{*}{\leftarrow}$ v $\rho$ are the obvious instances of $u \stackrel{*}{\rightarrow} u^{\prime}$ and $v^{\prime} \stackrel{*}{\leftarrow} v$, respectively.

Proof. It is clear from our assumption that $u \rho^{\prime} \stackrel{*}{\rightarrow} u^{\prime} \rho^{\prime} \mathbb{H}_{\mathcal{P}} u^{\prime} \rho \mathbb{H} V, \mathcal{P} v^{\prime} \rho \stackrel{*}{\leftarrow} v \rho$. Thus it remains to show $u^{\prime} \rho^{\prime} \mathbb{H} \mathcal{P} v^{\prime} \rho$. Since $\mathcal{V}_{V}\left(u^{\prime}\right) \subseteq X$ and $\operatorname{dom}(\rho) \cap X=\emptyset, \operatorname{dom}(\rho) \cap \mathcal{V}_{V}\left(u^{\prime}\right)=\emptyset$. Thus, since $u^{\prime} \mathbb{H}_{V, \mathcal{P}} v^{\prime}$, it follows that $u^{\prime} \rho^{\prime} \mathbb{H} \mathcal{P} v^{\prime} \rho$ by Lemma 3.22 .

Example 3.24. Suppose we have TRSs $\mathcal{S}=\{+(0, y) \rightarrow y,+(\mathrm{s}(x), y) \rightarrow \mathbf{s}(+(x, y))\}$ and $\mathcal{P}=\{\mathrm{s}(\mathrm{s}(x)) \rightarrow \mathrm{s}(x)\}$. Then we have $\left\langle+(\mathrm{s}(x), y), \mathrm{s}(+(\mathrm{s}(x), y)\rangle_{\{x\}} \in \mathrm{PCP}_{i n}(\mathcal{P}, \mathcal{S})\right.$. This critical pair is joinable as $+(\mathrm{s}(x), y) \rightarrow \mathcal{S} \mathrm{s}(+(x, y))=u^{\prime} \mathbb{H}\{\epsilon\}, \mathcal{P} v^{\prime}=\mathrm{s}(\mathrm{s}(+(x, y))) \leftarrow \mathcal{S}$ $\mathrm{s}(+(\mathrm{s}(x), y))$. However, since $\mathcal{V}_{V}\left(u^{\prime}\right)=\mathcal{V}_{\{\epsilon\}}(\mathrm{s}(+(x, y)))=\{x, y\} \nsubseteq X=\{x\}$, the variable condition of Lemma 3.23 is not satisfied. Take $\rho, \rho^{\prime} \operatorname{such}$ that $\operatorname{dom}(\rho)=\operatorname{dom}\left(\rho^{\prime}\right)=\{y\}$, $\rho(y)=\mathrm{s}(\mathrm{s}(z))$ and $\rho^{\prime}(y)=\mathrm{s}(z)$. Then we have $\rho \rightarrow \mathcal{P} \rho^{\prime}$. Now, we can construct a rewrite sequence $+(\mathrm{s}(x), y) \rho^{\prime}=+(\mathrm{s}(x), \mathrm{s}(z)) \rightarrow \mathcal{S} \mathrm{s}(+(x, \mathrm{~s}(z))) \stackrel{*}{\leftarrow} \mathcal{P} \mathrm{s}(\mathrm{s}(+(x, \mathrm{~s}(\mathrm{~s}(z))))) \leftarrow \mathcal{S}$ $\mathrm{s}(+(\mathrm{s}(x), \mathrm{s}(\mathrm{s}(z))))=\mathrm{s}(+(\mathrm{s}(x), y)) \rho$. However, $\mathrm{s}(+(x, \mathrm{~s}(z))$ Ht $\mathcal{P} \mathrm{s}(\mathrm{s}(+(x, \mathrm{~s}(\mathrm{~s}(z)))))$ does not hold, as the two redex occurrences are not parallel.

Remark 3.25. The variable condition $\mathcal{V}_{V}\left(u^{\prime}\right) \subseteq X$ in Lemma 3.23 is not equivalent to $\mathcal{V}_{V}\left(v^{\prime}\right) \subseteq X$ if there exists $l \rightarrow r \in \mathcal{P}$ such that $\mathcal{V}(r) \subsetneq \mathcal{V}(l)$. In the sequel, however, $\mathcal{V}(r)=\mathcal{V}(l)$ for all $l \rightarrow r \in \mathcal{P}$ holds whenever we apply the lemma.

We extend Lemma 3.16 as follows.

Lemma 3.26. Let $\mathcal{Q}, \mathcal{R}$ be $T R S$ s such that $\mathcal{Q}$ is bidirectional and $\mathcal{R}$ is left-linear. Let be rewrite relations such that $s_{i} \triangleright t_{i}$ and $s_{j} \leftarrow_{\mathcal{Q}} t_{j}$ for any $j \in\{1, \ldots, n\} \backslash\{i\}$ implies $C\left[s_{1}, \ldots, s_{n}\right] \triangleright C\left[t_{1}, \ldots, t_{n}\right]$. Suppose $(i)$ for any $\langle u, v\rangle_{X} \in \mathrm{PCP}_{i n}(\mathcal{Q}, \mathcal{R})$ and substitutions $\rho, \rho^{\prime}$ such that $\rho \mathbb{H}_{\mathcal{Q}} \rho^{\prime}$ and $\operatorname{dom}(\rho) \cap X=\emptyset$, we have $u \rho^{\prime}-v \rho$ and (ii) $\operatorname{CP}(\mathcal{R}, \mathcal{Q}) \subseteq 4$. If $\stackrel{+}{\rightarrow}_{\mathcal{R}} \circ \mathbb{H}_{\mathcal{Q}} \circ \stackrel{*}{\leftarrow}_{\mathcal{R}} \subseteq$ then $\mathbb{H}{ }_{\mathcal{Q}} \circ \rightarrow_{\mathcal{R}} \subseteq \boldsymbol{\vee}$.

Proof. Suppose $t_{0} \mathbb{H}_{U, \mathcal{Q}} s \rightarrow_{q, \mathcal{R}} t_{1}$. Let $U=\left\{p_{1}, \ldots, p_{n}\right\}$ where $p_{1}, \ldots, p_{n}$ are positions from left to right, $s / p_{i}=l_{i} \sigma_{i}$ for $l_{i} \rightarrow r_{i} \in \mathcal{Q}$ and substitutions $\sigma_{i}(1 \leq i \leq n)$ and $s / q=l^{\prime} \rho$ for $l^{\prime} \rightarrow r^{\prime} \in \mathcal{R}$ and substitution $\rho$. The same proof as in Lemma 3.16 applies other than 
the case of $\forall p \in U . p \not \leq q$. Let $\left\{p_{k}, \ldots, p_{m}\right\}=\left\{p_{i} \in U \mid q \leq p_{i}\right\}$. For each $p_{i}(k \leq i \leq m)$ either $p_{i} / q \in \operatorname{Pos}_{\mathcal{F}}\left(l^{\prime}\right)$ or there exists $q_{x} \in \operatorname{Pos}_{\mathcal{V}}\left(l^{\prime}\right)$ such that $q . q_{x} \leq p_{i}$. W.l.o.g. let $\left\{p_{k}, \ldots, p_{l}\right\}=\left\{p_{i} \mid p_{i} / q \in \operatorname{Pos}_{\mathcal{F}}\left(l^{\prime}\right)\right\}$ and $\left\{p_{l+1}, \ldots, p_{m}\right\}=\left\{p_{i} \mid \exists q_{x} \in \operatorname{Pos} \mathcal{V}\left(l^{\prime}\right) . q \cdot q_{x} \leq p_{i}\right\}$. Then there exists a parallel critical pair $\langle u, v\rangle_{X}$ obtained from overlaps of $l_{k} \rightarrow r_{k}, \ldots, l_{l} \rightarrow r_{l}$ on $l^{\prime} \rightarrow r^{\prime}$ at $p_{k} / q, \ldots, p_{l} / q$. Since $l^{\prime}$ is linear (and $\mathcal{V}\left(l^{\prime}\right), \mathcal{V}\left(l_{1}\right), \ldots, \mathcal{V}\left(l_{m}\right)$ are mutually disjoint), $t_{0} / q=u \rho^{\prime}$ and $t_{1} / q=v \rho$ for some substitutions $\rho, \rho^{\prime}$ such that $\rho \boxplus \mathcal{Q} \rho^{\prime}$ and $\operatorname{dom}(\rho) \cap X=\emptyset$. Hence by our assumption $u \rho^{\prime} \vee v \rho$. Thus by our assumption on $\boldsymbol{\nabla}$, it follows that $t_{0}=s\left[r_{1} \sigma_{1}, \ldots, r_{k-1} \sigma_{k-1}, u \rho^{\prime}, r_{m+1} \sigma_{m+1}, \ldots, r_{n} \sigma_{n}\right]_{p_{1}, \ldots, p_{k-1}, q, p_{l+1}, \ldots, p_{n}}$ $s\left[l_{1} \sigma_{1}, \ldots, l_{k-1} \sigma_{k-1}, v \rho, l_{m+1} \sigma_{m+1}, \ldots, l_{n} \sigma_{n}\right]_{p_{1}, \ldots, p_{k-1}, q, p_{l+1}, \ldots, p_{n}}=t_{1}$.

The following lemma is analogous to Lemmas 3.7, 3.17 .

Lemma 3.27. Let $\mathcal{P}, \mathcal{S}, \mathcal{P}^{\prime}$ be TRSs such that $\mathcal{P}$ is bidirectional and $\mathcal{S}$ is left-linear. Let

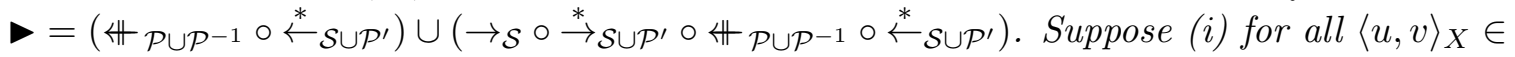
$\mathrm{PCP}_{\text {in }}\left(\mathcal{P} \cup \mathcal{P}^{-1}, \mathcal{S}\right)$, either $u=u^{\prime} \mathbb{H}_{V, \mathcal{P} \cup \mathcal{P}^{-1}} \circ \stackrel{*}{\leftarrow} \mathcal{S} \cup \mathcal{P}^{\prime}$ v or $u \rightarrow_{\mathcal{S}} \circ \stackrel{*}{\rightarrow}_{\mathcal{S} \cup \mathcal{P}^{\prime}} u^{\prime} \mathbb{H}_{V, \mathcal{P} \cup \mathcal{P}^{-1}}$ $\circ \stackrel{*}{\leftarrow} \mathcal{S} \cup \mathcal{P}^{\prime} v$ for some $u^{\prime}$ and $V$ satisfying $\mathcal{V}_{V}\left(u^{\prime}\right) \subseteq X$ and $\left(\right.$ ii) $\operatorname{CP}\left(\mathcal{S}, \mathcal{P} \cup \mathcal{P}^{-1}\right) \subseteq \varangle$. Then $\mathbb{H}_{\mathcal{P} \cup \mathcal{P}^{-1}} \circ \rightarrow \mathcal{S} \subseteq \triangleright$.

Proof. Take $\mathcal{Q}:=\mathcal{P} \cup \mathcal{P}^{-1}$, which is a bidirectional TRS by bidirectionality of $\mathcal{P}$, and $\mathcal{R}:=\mathcal{S}$ in Lemma 3.26. Then by the condition (ii) we have $\operatorname{CP}(\mathcal{R}, \mathcal{Q}) \subseteq \varangle$. Furthermore, by the condition (i) and Lemma 3.23, for any $\langle u, v\rangle_{X} \in \mathrm{PCP}_{i n}(\mathcal{Q}, \mathcal{R})$ and substitutions $\rho, \rho^{\prime}$ such that $\rho \mathbb{R}_{\mathcal{Q}} \rho^{\prime}$ and $\operatorname{dom}(\rho) \cap X=\emptyset$, we have either $u \rho^{\prime}=u^{\prime} \rho^{\prime} \mathbb{H} \mathcal{P} \cup \mathcal{P}^{-1} \circ \stackrel{*}{\leftarrow} \mathcal{S} \cup \mathcal{P}^{\prime}$ $v \rho$ or $u \rho^{\prime} \rightarrow \mathcal{S} \circ \stackrel{*}{\rightarrow} \mathcal{S} \cup \mathcal{P}^{\prime} u^{\prime} \rho^{\prime} \mathbb{H}_{\mathcal{P} \cup \mathcal{P}^{-1}} \circ \stackrel{*}{\leftarrow} \mathcal{S} \cup \mathcal{P}^{\prime} v \rho$ and hence $u \rho^{\prime} \vee v \rho$. We also have

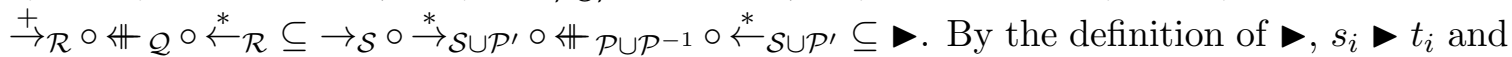
$s_{j} \leftarrow \mathcal{P} \cup \mathcal{P}^{-1} t_{j}$ for any $j \in\{1, \ldots, n\} \backslash\{i\}$ implies $C\left[s_{1}, \ldots, s_{n}\right] \triangleright C\left[t_{1}, \ldots, t_{n}\right]$. Hence the claim follows from Lemma 3.26 .

The next theorem strengthens Theorem 3.18 .

Theorem 3.28. Let $\mathcal{P}, \mathcal{S}, \mathcal{P}^{\prime}$ be TRSs such that $\mathcal{S}$ is left-linear, $\mathcal{P}$ is reversible, $\mathcal{P}^{\prime} \subseteq$

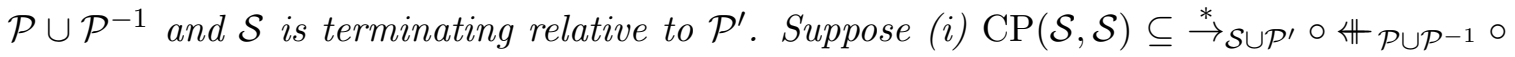
$\stackrel{*}{\leftarrow} \mathcal{S} \cup \mathcal{P}^{\prime}$, (ii) for all $\langle u, v\rangle_{X} \in \mathrm{PCP}_{i n}\left(\mathcal{P} \cup \mathcal{P}^{-1}, \mathcal{S}\right)$, either $u=u^{\prime} \|_{V, \mathcal{P} \cup \mathcal{P}^{-1}} \circ \stackrel{*}{\leftarrow} \mathcal{S} \cup \mathcal{P}^{\prime} v$ or

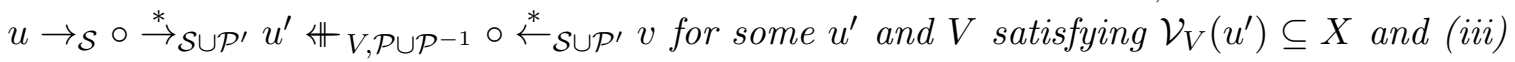
$\mathrm{CP}\left(\mathcal{S}, \mathcal{P} \cup \mathcal{P}^{-1}\right) \subseteq\left(\stackrel{*}{\rightarrow}_{\mathcal{S} \cup \mathcal{P}^{\prime}} \circ \mathbb{H}_{\mathcal{P} \cup \mathcal{P}^{-1}}\right) \cup\left(\stackrel{*}{\rightarrow}_{\mathcal{S} \cup \mathcal{P}^{\prime}} \circ \#_{\mathcal{P} \cup \mathcal{P}^{-1}} \circ \stackrel{*}{\leftarrow} \mathcal{S} \cup \mathcal{P}^{\prime} \circ \leftarrow \mathcal{S}\right)$. Then $\mathcal{S} \cup \mathcal{P}$ is confluent.

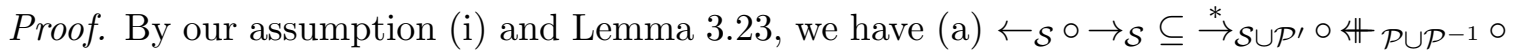

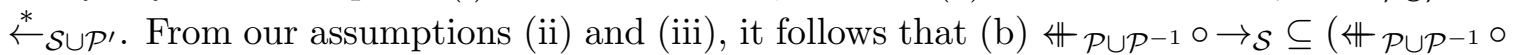

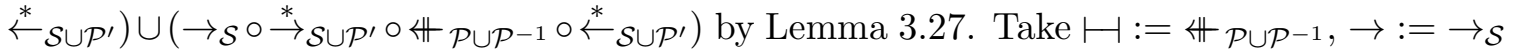
and $\leadsto:=\rightarrow \mathcal{P}^{\prime}$. Then, by the termination of $\mathcal{S}$ relative to $\mathcal{P}^{\prime}, \rightarrow \circ \stackrel{*}{\sim}$ is well-founded. Thus

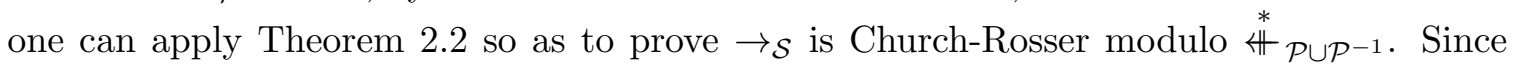
$\stackrel{*}{\mathbb{H}_{\mathcal{P}} \cup \mathcal{P}^{-1}}=\stackrel{*}{\leftrightarrow} \mathcal{P}$, it follows that $\rightarrow_{\mathcal{S}}$ is Church-Rosser modulo $\stackrel{*^{\prime}}{\leftrightarrow} \mathcal{P}$. Hence, since $\rightarrow_{\mathcal{P}}$ is reversible, $\rightarrow \mathcal{S} \cup \mathcal{P}$ is confluent by Lemma 3.3 .

By the definition of parallel critical pairs, $\mathrm{CP}_{\text {in }}\left(\mathcal{P} \cup \mathcal{P}^{-1}, \mathcal{S}\right)=\emptyset$ implies $\operatorname{PCP}_{\text {in }}(\mathcal{P} \cup$ $\left.\mathcal{P}^{-1}, \mathcal{S}\right)=\emptyset$. Thus the condition (ii) of Theorem 3.18 is a particular case of condition (ii) of Theorem 3.28. Hence Theorem 3.18 is subsumed by Theorem 3.28 .

By taking $\mathcal{P}^{\prime}=\emptyset$ in Theorem 3.28 , we obtain the next corollary. 
Corollary 3.29. Let $\mathcal{P}, \mathcal{S}$ be TRSs such that $\mathcal{S}$ is left-linear, $\mathcal{P}$ is reversible and $\mathcal{S}$ is terminating. Suppose (i) $\mathrm{CP}(\mathcal{S}, \mathcal{S}) \subseteq \stackrel{*}{\rightarrow} \mathcal{S}^{\prime} \circ \mathbb{H} \mathcal{P} \cup \mathcal{P}^{-1} \circ \stackrel{*}{\leftarrow} \mathcal{S}$, (ii) for all $\langle u, v\rangle_{X} \in \mathrm{PCP}_{\text {in }}(\mathcal{P} \cup$ $\left.\mathcal{P}^{-1}, \mathcal{S}\right), u \stackrel{*}{\rightarrow} \mathcal{S}^{\prime} u^{\prime} \|_{V, \mathcal{P} \cup \mathcal{P}^{-1}} \circ \stackrel{*}{\leftarrow} \mathcal{S} v$ for some $u^{\prime}$ and $V$ satisfying $\mathcal{V}_{V}\left(u^{\prime}\right) \subseteq X$ and (iii) $\mathrm{CP}\left(\mathcal{S}, \mathcal{P} \cup \mathcal{P}^{-1}\right) \subseteq \stackrel{*}{\rightarrow} \mathcal{S} \circ \boxplus \mathcal{P} \cup \mathcal{P}^{-1} \circ \stackrel{*}{\leftarrow} \mathcal{S}$. Then $\mathcal{S} \cup \mathcal{P}$ is confluent.

Considering a particular case of the condition (ii), we obtain the following corollary.

Corollary 3.30 (Theorem 2 of [10]). Let $\mathcal{P}, \mathcal{S}$ be TRSs such that $\mathcal{S}$ is left-linear, $\mathcal{P}$ is

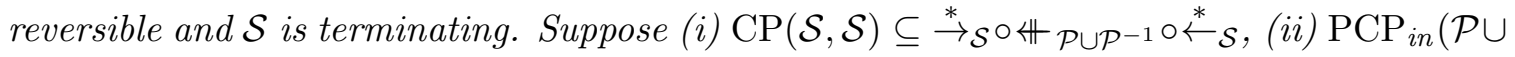
$\left.\mathcal{P}^{-1}, \mathcal{S}\right) \subseteq \stackrel{*}{\rightarrow} \mathcal{S} \circ \stackrel{*}{\leftarrow}^{S}$ and $\left(\right.$ iii) $\mathrm{CP}\left(\mathcal{S}, \mathcal{P} \cup \mathcal{P}^{-1}\right) \subseteq \stackrel{*}{\rightarrow} \mathcal{S} \circ \boxplus \mathcal{P} \cup \mathcal{P}-1 \circ \stackrel{*}{\leftarrow} \mathcal{S}$. Then $\mathcal{S} \cup \mathcal{P}$ is confluent.

3.5. Examples and comparison. In this subsection, some examples to illustrate applicability of our confluence criteria in previous subsections are presented. Relations among our confluence criteria and locations of given examples are summarized in Figure 1.

Example 3.31. Let $\mathcal{R}_{3}=\left\{\left(\operatorname{add}_{1}\right),\left(\operatorname{add}_{2}\right),(C),(A)\right\} \cup$

$$
\left\{\begin{array}{ll}
\left(\operatorname{add}_{3}\right)+(x, 0) & \rightarrow x \\
\left(\operatorname{add}_{4}\right)+(x, \mathrm{~s}(y)) & \rightarrow \mathrm{s}(+(x, y))
\end{array}\right\}
$$

where $\left(\operatorname{add}_{1}\right),\left(\operatorname{add}_{2}\right),(C),(A)$ are rewrite rules given in Example 1.2. We now prove confluence of $\mathcal{R}_{3}$ using Theorem 3.18. For this, put $\mathcal{S}=\left\{\left(\operatorname{add}_{1}\right),\left(\operatorname{add}_{2}\right),\left(\operatorname{add}_{3}\right),\left(\operatorname{add}_{4}\right)\right\}$ and $\mathcal{P}=\{(C),(A)\}$. Then $\mathcal{S}$ is linear and terminating. As demonstrated in Example $1.2, \mathcal{P}$ is reversible. We have $\mathrm{CP}(\mathcal{S}, \mathcal{S})=$

$$
\begin{aligned}
& \left\{\begin{array}{llll}
\langle 0,0\rangle & \in \stackrel{*}{\leftarrow} \mathcal{S}^{*} & \langle\mathrm{~s}(y), \mathrm{s}(+(0, y))\rangle & \in \leftarrow \mathcal{S} \\
\langle\mathrm{s}(+(x, 0)), \mathrm{s}(x)\rangle & \in \rightarrow \mathcal{S} & \langle\mathrm{s}(x), \mathrm{s}(+(x, 0))\rangle & \in \leftarrow \mathcal{S} \\
\langle\mathrm{s}(+(0, y)), \mathrm{s}(y)\rangle & \in \rightarrow \mathcal{S} & \langle\mathrm{s}(+(x, \mathrm{~s}(y))), \mathrm{s}(+(\mathrm{s}(x), y))\rangle \in \rightarrow \mathcal{S} \circ \leftarrow \mathcal{S} \\
\langle\mathrm{s}(+(\mathrm{s}(x), y)), \mathrm{s}(+(x, \mathrm{~s}(y)))\rangle \in \rightarrow \mathcal{S} \circ \leftarrow \mathcal{S} &
\end{array}\right\}, \\
& \mathrm{CP}_{i n}\left(\mathcal{P} \cup \mathcal{P}^{-1}, \mathcal{S}\right)=\emptyset \text { and } \operatorname{CP}\left(\mathcal{S}, \mathcal{P} \cup \mathcal{P}^{-1}\right)=
\end{aligned}
$$

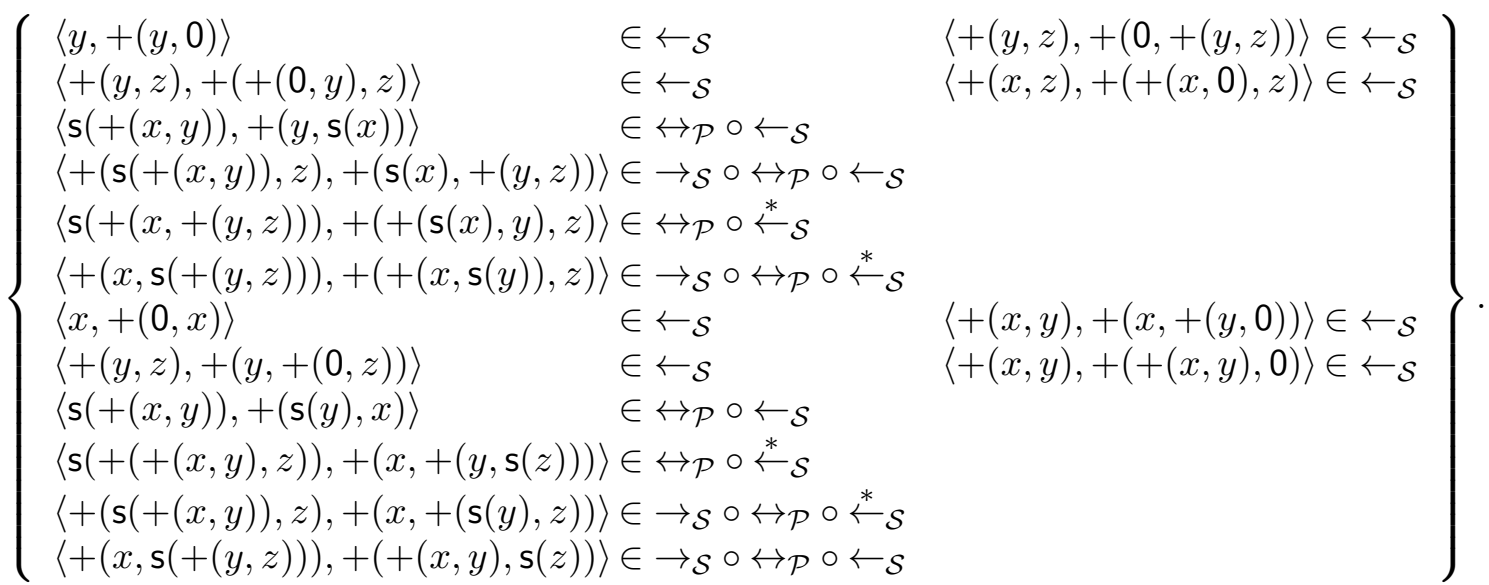

Thus one can apply Theorem 3.18 to obtain the confluence of $\mathcal{R}_{3}=\mathcal{S} \cup \mathcal{P}$. To this example, one can apply Theorem 3.8, Corollaries $3.10,3.19$ and Proposition 3.13 as well. 


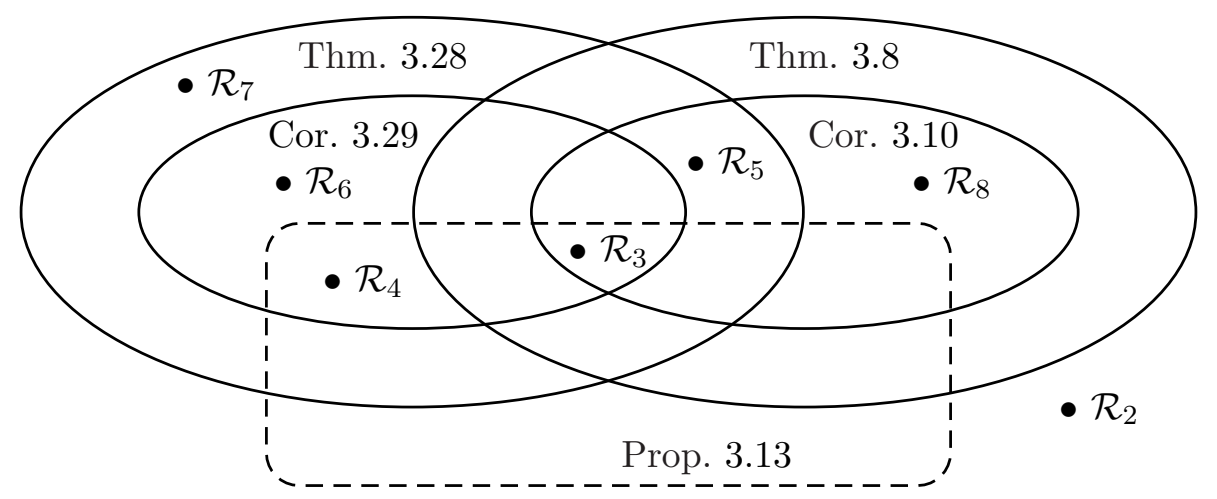

Figure 1: Relations among confluence criteria

Example 3.32. Let us consider $\mathcal{R}_{2}$ of Example 1.2. For this, we consider a partition $\mathcal{S}=$ $\left\{\left(\operatorname{add}_{1}\right),\left(\operatorname{add}_{2}\right)\right\}$ and $\mathcal{P}=\{(C),(A)\}$. Then $\mathcal{S}$ is linear and terminating and $\mathcal{P}$ is reversible. But there exists a critical pair $\langle y,+(y, 0)\rangle \in \operatorname{CP}\left(\mathcal{S}, \mathcal{P} \cup \mathcal{P}^{-1}\right)$ obtained by the overlap of

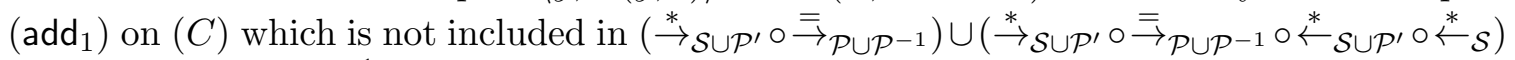
for any $\mathcal{P}^{\prime} \subseteq \mathcal{P} \cup \mathcal{P}^{-1}$. Thus the critical pair conditions of Theorem 3.8 are not satisfied. Since $\langle y,+(y, 0)\rangle$ is obtained by the overlap of $\left(\operatorname{add}_{1}\right)$ on $(C)$, another possible partition $\mathcal{S}=\left\{\left(\operatorname{add}_{1}\right),\left(\operatorname{add}_{2}\right),(A)\right\}$ and $\mathcal{P}=\{(C)\}$ is not effective either. Similarly, Theorem 3.28 and Proposition 3.13 are also not applicable. We will revisit this example in the next section.

Example 3.33. Let

$$
\mathcal{R}_{4}=\mathcal{R}_{3} \cup\{(\mathrm{dbl}) \quad \mathrm{dbl}(x) \rightarrow+(x, x)\} .
$$

One can easily confirm that the confluence of $\mathcal{R}_{1}$ is shown in the same way as $\mathcal{R}_{3}$ using Theorem 3.18 by putting $\mathcal{S}=\left\{\left(\operatorname{add}_{1}\right),\left(\operatorname{add}_{2}\right),\left(\operatorname{add}_{3}\right),\left(\operatorname{add}_{4}\right),(\mathrm{dbl})\right\}$ and $\mathcal{P}=\{(C),(A)\}$. On the other hand, since $\mathcal{S}$ is not linear, Theorem 3.8 does not apply. To this example, one can apply Corollary 3.19 and Proposition 3.13 as well.

Example 3.34. Consider a TRS

$$
\mathcal{R}_{5}=\mathcal{R}_{3} \cup\left\{\begin{array}{lll}
\left(\mathrm{ss}_{1}\right) & \mathrm{s}(x) & \rightarrow \mathrm{s}(\mathrm{s}(x)) \\
\left(\mathrm{ss}_{2}\right) & \mathrm{s}(\mathrm{s}(x)) & \rightarrow \mathrm{s}(x)
\end{array}\right\} .
$$

By putting $\mathcal{S}=\left\{\left(\operatorname{add}_{1}\right),\left(\operatorname{add}_{2}\right),\left(\operatorname{add}_{3}\right),\left(\operatorname{add}_{4}\right)\right\}$ and $\mathcal{P}=\left\{(C),(A),\left(\mathrm{ss}_{1}\right),\left(\mathrm{ss}_{2}\right)\right\}$, one can show the confluence of $\mathcal{R}_{5}$ using Corollary 3.10. On the other hand, the condition of Corollary 3.29 is not satisfied because $\mathrm{CP}_{i n}\left(\mathcal{P} \cup \mathcal{P}^{-1}, \mathcal{S}\right) \neq \emptyset$. Proposition 3.13 does not apply either, since $\mathcal{S}$ is not terminating relative to $\mathcal{P}$ as e.g. $+(\mathrm{s}(\mathrm{s}(x)), y) \rightarrow \mathcal{S} \mathrm{s}(+(\mathrm{s}(x), y)) \rightarrow \mathcal{P}$ $\mathrm{s}(+(\mathrm{s}(\mathrm{s}(x)), y)) \rightarrow_{\mathcal{S}} \cdots$. Take $\mathcal{P}^{\prime}=\left\{\left(\mathrm{ss}_{2}\right)\right\}$. Then the conditions of Theorem 3.28 are satisfied - for example, for $\langle+(\mathrm{s}(\mathrm{s}(x)), y), \mathrm{s}(+(x, y))\rangle_{\{x\}} \in \mathrm{PCP}_{i n}\left(\mathcal{P} \cup \mathcal{P}^{-1}, \mathcal{S}\right)$, we have a

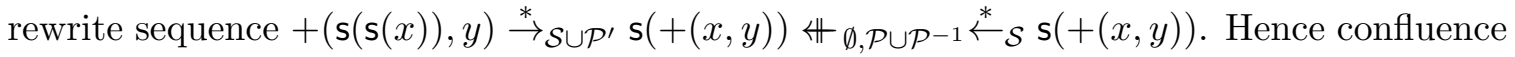
of $\mathcal{R}_{5}$ is shown by Theorem 3.28 .

Example 3.35. Let

$$
\mathcal{R}_{6}=\left\{\left(\operatorname{add}_{1}\right),\left(\operatorname{add}_{2}\right),\left(\operatorname{add}_{3}\right),(C),(A),(\mathrm{dbl})\right\} \cup\left\{\left(\operatorname{add}_{5}\right)+(x, \mathrm{~s}(y)) \rightarrow+(\mathrm{s}(x), y)\right\} .
$$


Let $\mathcal{S}=\left\{\left(\operatorname{add}_{1}\right),\left(\operatorname{add}_{2}\right),\left(\operatorname{add}_{3}\right),\left(\operatorname{add}_{5}\right),(\mathrm{dbl})\right\}$ and $\mathcal{P}=\{(C),(A)\}$. Then $\mathcal{S}$ is not terminating relative to $\mathcal{P}$, because of $+(x, \mathrm{~s}(x)) \rightarrow_{\mathcal{S}}+(\mathrm{s}(x), x) \rightarrow_{\mathcal{P}}+(x, \mathrm{~s}(x)) \rightarrow_{\mathcal{S}} \cdots$. Thus Proposition 3.13 is not applicable. Since $\mathcal{S}$ is not linear, Theorem 3.8 does not apply either. On the other hand, one easily checks the confluence of $\mathcal{R}_{6}$ using Theorems 3.18 or 3.28 . To this example, Corollaries 3.19 and 3.29 also apply.

Example 3.36. Let

$$
\mathcal{R}_{7}=\mathcal{R}_{4} \cup \mathcal{R}_{5}=\left\{\left(\operatorname{add}_{1}\right),\left(\operatorname{add}_{2}\right),\left(\operatorname{add}_{3}\right),\left(\operatorname{add}_{4}\right),(C),(A),(\mathrm{dbl}),\left(\mathrm{ss}_{1}\right),\left(\mathrm{ss}_{2}\right)\right\} .
$$

By the same reason as for $\mathcal{R}_{6}$, Proposition 3.13 and Theorem 3.8 do not apply. By the same reason as for $\mathcal{R}_{5}$, Corollary 3.29 does not apply. Confluence of $\mathcal{R}_{7}$ can be shown as in Example 3.34 by taking $\left.\mathcal{S}=\left(\operatorname{add}_{1}\right),\left(\operatorname{add}_{2}\right),\left(\operatorname{add}_{3}\right),\left(\operatorname{add}_{4}\right),(\mathrm{dbl})\right\}, \mathcal{P}=\left\{(C),(A),\left(\mathrm{ss}_{1}\right),\left(\mathrm{ss}_{2}\right)\right\}$ and $\mathcal{P}^{\prime}=\left\{\left(\mathrm{ss}_{2}\right)\right\}$.

Example 3.37. Let

$$
\mathcal{R}_{8}=\left\{\begin{array}{llll}
(a) \mathrm{f}(\mathrm{g}(x), \mathrm{g}(y)) & \rightarrow \mathrm{f}(\mathrm{g}(x), \mathrm{h}(y)) & (b) \mathrm{f}(\mathrm{h}(x), \mathrm{g}(y)) & \rightarrow \mathrm{f}(\mathrm{g}(x), \mathrm{g}(y)) \\
(c) \mathrm{f}(\mathrm{g}(x), \mathrm{h}(y)) & \rightarrow \mathrm{f}(x, y) & (d) \mathrm{f}(\mathrm{h}(x), \mathrm{h}(y)) & \rightarrow \mathrm{f}(y, x) \\
(e) \mathrm{f}(x, y) & \rightarrow \mathrm{f}(y, x) & (f) \mathrm{g}(x) & \rightarrow \mathrm{h}(x) \\
(g) \mathrm{h}(x) & \rightarrow \mathrm{g}(x) & &
\end{array}\right\} .
$$

Let $\mathcal{S}=\{(a),(b),(c),(d)\}$ and $\mathcal{P}=\{(e),(f),(g)\}$. We have $\operatorname{CP}(\mathcal{S}, \mathcal{S})=\emptyset, \mathrm{CP}_{\text {in }}(\mathcal{P} \cup$ $\left.\mathcal{P}^{-1}, \mathcal{S}\right)=$

$$
\left\{\begin{array}{llll}
\langle\mathrm{f}(\mathrm{h}(x), \mathrm{g}(y)), \mathrm{f}(\mathrm{g}(y), \mathrm{h}(x))\rangle & \in \leftrightarrow_{\mathcal{P}} & \langle\mathrm{f}(\mathrm{g}(x), \mathrm{h}(y)), \mathrm{f}(\mathrm{g}(x), \mathrm{h}(y))\rangle \in i d \\
\langle\mathrm{f}(\mathrm{g}(x), \mathrm{g}(y)), \mathrm{f}(\mathrm{g}(x), \mathrm{g}(y))\rangle & \in i d & & \\
\langle\mathrm{f}(\mathrm{h}(x), \mathrm{h}(y)), \mathrm{f}(\mathrm{g}(x), \mathrm{g}(y))\rangle & \in \rightarrow_{\mathcal{S}} \circ \leftrightarrow_{\mathcal{P}} \circ \stackrel{\leftarrow}{\leftarrow}_{\mathcal{S}} & \\
\langle\mathrm{f}(\mathrm{h}(x), \mathrm{h}(y)), \mathrm{f}(x, y)\rangle & \in \rightarrow \mathcal{S} \circ \leftrightarrow_{\mathcal{P}} & \langle\mathrm{f}(\mathrm{g}(x), \mathrm{g}(y)), \mathrm{f}(x, y)\rangle & \in \stackrel{*}{\rightarrow} \\
\langle\mathrm{f}(\mathrm{g}(x), \mathrm{h}(y)), \mathrm{f}(y, x)\rangle & \in \rightarrow \mathcal{S} \circ \leftrightarrow_{\mathcal{P}} & \langle\mathrm{f}(\mathrm{h}(x), \mathrm{g}(y)), \mathrm{f}(y, x)\rangle & \in \stackrel{\rightarrow}{\mathcal{S}}_{\mathcal{S}} \circ \leftrightarrow_{\mathcal{P}}
\end{array}\right\}
$$

and $\operatorname{CP}\left(\mathcal{S}, \mathcal{P} \cup \mathcal{P}^{-1}\right)=$

$$
\left\{\begin{array}{llll}
\langle\mathrm{f}(\mathrm{g}(x), \mathrm{g}(y)), \mathrm{f}(\mathrm{g}(y), \mathrm{h}(x))\rangle & \in \leftrightarrow_{\mathcal{P}} & \langle\mathrm{f}(\mathrm{g}(x), \mathrm{h}(y)), \mathrm{f}(\mathrm{g}(y), \mathrm{g}(x))\rangle & \in \leftrightarrow_{\mathcal{P}} \\
\langle\mathrm{f}(x, y), \mathrm{f}(\mathrm{h}(y), \mathrm{g}(x))\rangle & \in \leftarrow^{*} \mathcal{S} & \langle\mathrm{f}(x, y), \mathrm{f}(\mathrm{h}(x), \mathrm{h}(y))\rangle & \in \leftrightarrow_{\mathcal{P}} \circ \leftarrow_{\mathcal{S}}
\end{array}\right\} .
$$

Thus $\mathcal{R}_{8}$ is confluent by Corollary 3.10 . Proposition 3.13 does not apply, since $\mathcal{S}$ is not terminating relative to $\mathcal{P}$. Furthermore, the conditions of Theorem 3.28 are not satisfied. For $\langle\mathrm{f}(\mathrm{h}(x), \mathrm{h}(y)), \mathrm{f}(x, y)\rangle_{\{x\}} \in \mathrm{PCP}_{i n}\left(\mathcal{P} \cup \mathcal{P}^{-1}, \mathcal{S}\right)$, the critical pair conditions can not be satisfied. For, any rewrite sequence $\mathrm{f}(\mathrm{h}(x), \mathrm{h}(y)) \stackrel{*}{\rightarrow}_{\mathcal{S} \cup \mathcal{P}^{\prime}} \circ \mathbb{H}_{V, \mathcal{P} \cup \mathcal{P}^{-1}} \circ \stackrel{*}{\leftarrow} \mathcal{S} \cup \mathcal{P}^{\prime} \mathrm{f}(x, y)$ satisfying the critical pair conditions, we have $\mathrm{f}(x, y) \rightarrow \mathrm{f}(y, x) \in \mathcal{P}^{\prime}$. (Note that if we take $\mathcal{P}^{\prime}:=\emptyset$ then the rewrite sequence $\mathrm{f}(\mathrm{h}(x), \mathrm{h}(y)) \stackrel{*}{\rightarrow}_{\mathcal{S}} \mathrm{f}(y, x) \mathbb{H}_{\{\epsilon\}, \mathcal{P} \cup \mathcal{P}^{-1}} \mathrm{f}(x, y)$ does not satisfy the critical pair conditions because of the variable condition as $\mathcal{V}_{\{\epsilon\}}(\mathrm{f}(y, x))=$ $\{x, y\} \nsubseteq\{x\}$.) But then $\mathcal{S}$ is not terminating relative to $\mathcal{P}^{\prime}$.

\section{Theorem 3.38.}

(1) Corollary 3.10 and Theorem 3.28 are incomparable.

(2) Corollary 3.29 and Theorem 3.8 are incomparable.

(3) Theorems 3.8 and 3.28 are incomparable.

(4) Corollaries 3.10 and 3.29 are incomparable.

Proof. Each claim is witnessed by the preceding examples. See Figure 1 . 


$$
\begin{aligned}
& \text { Partition } \frac{\langle\mathcal{S}, \mathcal{P}\rangle}{\left\langle\mathcal{S}^{\prime}, \mathcal{P}^{\prime}\right\rangle} \mathcal{S} \cup \mathcal{P}=\mathcal{S}^{\prime} \cup \mathcal{P}^{\prime}, \mathcal{P}^{\prime} \text { : reversible } \\
& \text { Replacement } \quad \frac{\langle\mathcal{S} \cup\{l \rightarrow r\}, \mathcal{P}\rangle}{\left\langle\mathcal{S} \cup\left\{l \rightarrow r^{\prime}\right\}, \mathcal{P}\right\rangle} r \stackrel{*}{\leftrightarrow} \mathcal{P} r^{\prime} \\
& \text { Addition } \frac{\langle\mathcal{S}, \mathcal{P}\rangle}{\langle\mathcal{S} \cup\{l \rightarrow r\}, \mathcal{P}\rangle} l \stackrel{*}{\leftrightarrow} \mathcal{P} \circ \stackrel{*}{\rightarrow} \mathcal{S} r
\end{aligned}
$$

Figure 2: Inference rules of reduction-preserving completion

\section{Reduction-PRESERVING COMPLETION}

In this section, we give a reduction-preserving completion procedure so that the applicability of the criteria presented in the previous section is enhanced. We first present an abstract procedure formulated in terms of inference rules (subsection 1) and then give a concrete one which forms a basis of our implementation (subsection 2).

4.1. Abstract completion procedure. As witnessed in Example 3.32, there are cases where our confluence criteria are not applicable directly. Our idea is to construct a TRS suitable for applying our criteria by exchanging or adding rewrite rules without changing the reduction relation so that the confluence of the transformed TRS implies that of the original TRS. Using the reversibility of $\mathcal{P}$ allows several flexibilities on such transformations.

The notion of reduction equivalence and the following properties of reduction equivalence are well-known in literature and the latter are easily proved.

Definition 4.1 (reduction equivalence). Two relations $\rightarrow_{0}$ and $\rightarrow_{1}$ are said to be reduction equivalent if $\stackrel{*}{\rightarrow}_{0}=\stackrel{*}{\rightarrow}_{1}$. Two TRSs $\mathcal{R}$ and $\mathcal{Q}$ are reduction equivalent if so are $\rightarrow_{\mathcal{R}}$ and $\rightarrow_{\mathcal{Q}}$

Proposition 4.2 (properties of reduction equivalence). (i) If $\rightarrow_{\mathcal{R}} \subseteq \stackrel{*}{\rightarrow}_{\mathcal{Q}}$ and $\rightarrow_{\mathcal{Q}} \subseteq \stackrel{*}{\rightarrow} \mathcal{R}$ then $\mathcal{R}$ and $\mathcal{Q}$ are reduction equivalent. (ii) If $\mathcal{R}$ and $\mathcal{Q}$ are reduction equivalent then the confluence of $\mathcal{R}$ and $\mathcal{Q}$ coincide.

We now demonstrate how the confluence criteria in the previous section can be applied indirectly using the notion of reduction equivalence.

Example 4.3 (confluence by reduction equivalence). We show the confluence of $\mathcal{R}_{2}=$ $\left\{\left(\operatorname{add}_{1}\right),\left(\operatorname{add}_{2}\right),(C),(A)\right\}$ of Example 1.2 . Theorems 3.28 and 3.8 can not be applied directly to prove this as illustrated in Example 3.32. Consider $\mathcal{R}_{3}=\mathcal{R}_{2} \cup\left\{\left(\operatorname{add}_{3}\right),\left(\operatorname{add}_{4}\right)\right\}$ of Example 3.31. Then since we have $+(x, 0) \rightarrow_{\mathcal{R}_{2}}+(0, x) \rightarrow_{\mathcal{R}_{2}} x$ and $+(x, \mathrm{~s}(y)) \rightarrow_{\mathcal{R}_{2}}$ $+(\mathrm{s}(y), x) \rightarrow_{\mathcal{R}_{2}} \mathrm{~s}(+(y, x)) \rightarrow_{\mathcal{R}_{2}} \mathrm{~s}(+(x, y))$, the inclusions $\rightarrow_{\mathcal{R}_{2}} \subseteq \rightarrow_{\mathcal{R}_{3}} \subseteq \stackrel{*}{\rightarrow}_{\mathcal{R}_{2}}$ hold. Hence $\mathcal{R}_{3}$ and $\mathcal{R}_{2}$ are reduction equivalent by Proposition 4.2 (i). As we have shown in Example 3.31, $\mathcal{R}_{3}$ is confluent. Thus by Proposition 4.2 (ii), $\mathcal{R}_{2}$ is confluent too.

In this example, two additional rewrite rules $\left(\operatorname{add}_{2}\right)$ and $\left(\operatorname{add}_{3}\right)$ are given by hand. But in automated confluence proving procedures, one needs to find such new rewrite rules automatically. We next present a completion-like procedure to automate such additions (or 
more generally transformations) of rewrite rules. We first present an abstract version of the procedure in the form of inference rules and prove its soundness w.r.t. the confluence proof.

Definition 4.4 (abstract reduction-preserving completion procedure). Inference rules of an abstract reduction-preserving completion procedure are listed in Figure 2, The inference rules act on a pair of TRSs $\mathcal{S}$ and $\mathcal{P}$ where $\mathcal{P}$ is reversible. One step derivation using any of inference rules (from upper to lower) is denoted by $\leadsto$. We also write $\sim^{p}\left(\sim^{r}, \sim^{a}\right)$ for an inference step by the rule Partition (Replacement, Addition, respectively).

Theorem 4.5 (soundness of the abstract reduction-preserving completion procedure). Let $\langle\mathcal{R}, \emptyset\rangle=\left\langle\mathcal{S}_{0}, \mathcal{P}_{0}\right\rangle \stackrel{*}{\sim}\left\langle\mathcal{S}_{n}, \mathcal{P}_{n}\right\rangle$ be a derivation of abstract reduction-preserving completion procedure. If $\mathcal{S}_{n} \cup \mathcal{P}_{n}$ is confluent then $\mathcal{R}$ is confluent.

Proof. We show, for any inference step $\left\langle\mathcal{S}_{i}, \mathcal{P}_{i}\right\rangle \leadsto\left\langle\mathcal{S}_{i+1}, \mathcal{P}_{i+1}\right\rangle$, that $\mathcal{S}_{i} \cup \mathcal{P}_{i}$ and $\mathcal{S}_{i+1} \cup \mathcal{P}_{i+1}$ are reduction equivalent and that $\mathcal{P}_{i+1}$ is reversible whenever so is $\mathcal{P}_{i}$.

- $\left\langle\mathcal{S}_{i}, \mathcal{P}_{i}\right\rangle \leadsto\left\langle\mathcal{S}_{i+1}, \mathcal{P}_{i+1}\right\rangle$ by Partition. Then since $\mathcal{S}_{i} \cup \mathcal{P}_{i}=\mathcal{S}_{i+1} \cup \mathcal{P}_{i+1}$ and $\mathcal{P}_{i+1}$ is reversible by the side condition, the claim follows immediately.

- $\left\langle\mathcal{S}_{i}, \mathcal{P}_{i}\right\rangle \leadsto\left\langle\mathcal{S}_{i+1}, \mathcal{P}_{i+1}\right\rangle$ by Replacement. Then $\mathcal{S}_{i}=\mathcal{S}_{i}^{\prime} \cup\{l \rightarrow r\}, r \stackrel{*}{\leftrightarrow} \mathcal{P}_{i} r^{\prime}$ and $\mathcal{S}_{i+1}=$ $\mathcal{S}_{i}^{\prime} \cup\left\{l \rightarrow r^{\prime}\right\}$ for some $\mathcal{S}_{i}^{\prime}, l, r, r^{\prime}$ and $\mathcal{P}_{i+1}=\mathcal{P}_{i}$. By the reversibility of $\mathcal{P}_{i}$, we have $l \rightarrow \mathcal{S}_{i} r \stackrel{*}{\rightarrow} \mathcal{P}_{i} r^{\prime}$ hence $\rightarrow \mathcal{S}_{i+1} \cup \mathcal{P}_{i+1} \subseteq \stackrel{*}{\rightarrow} \mathcal{S}_{i} \cup \mathcal{P}_{i}$. By the reversibility of $\mathcal{P}_{i}$, we also have $l \rightarrow \mathcal{S}_{i+1} r^{\prime} \stackrel{*}{\rightarrow}_{\mathcal{P}_{i}} r$, hence $\rightarrow \mathcal{S}_{i} \cup \mathcal{P}_{i} \subseteq \stackrel{*}{\rightarrow} \mathcal{S}_{i+1} \cup \mathcal{P}_{i+1}$. Thus by Proposition 4.2 (i), $\mathcal{S}_{i} \cup \mathcal{P}_{i}$ and $\mathcal{S}_{i+1} \cup \mathcal{P}_{i+1}$ are reduction equivalent. Hence, by $\mathcal{P}_{i+1}=\mathcal{P}_{i}$, the claim follows.

- $\left\langle\mathcal{S}_{i}, \mathcal{P}_{i}\right\rangle \leadsto\left\langle\mathcal{S}_{i+1}, \mathcal{P}_{i+1}\right\rangle$ by Addition. Then $l \stackrel{*}{\leftrightarrow} \mathcal{P}_{i} \circ \stackrel{*}{\rightarrow} \mathcal{S}_{i} r$ and $\mathcal{S}_{i+1}=\mathcal{S}_{i} \cup\{l \rightarrow r\}$ for some $l, r$ and and $\mathcal{P}_{i+1}=\mathcal{P}_{i}$. Since $\mathcal{S}_{i} \cup \mathcal{P}_{i} \subseteq \mathcal{S}_{i+1} \cup \mathcal{P}_{i+1}$, we have $\rightarrow \mathcal{S}_{i} \cup \mathcal{P}_{i} \subseteq \stackrel{*}{\rightarrow}_{\mathcal{S}_{i+1} \cup \mathcal{P}_{i+1}}$. By the reversibility of $\mathcal{P}_{i}, l \stackrel{*}{\rightarrow} \mathcal{P}_{i} \circ \stackrel{*}{\rightarrow} \mathcal{S}_{i} r^{\prime}$. Hence $\rightarrow \mathcal{S}_{i+1} \cup \mathcal{P}_{i+1} \subseteq \stackrel{*}{\rightarrow} \mathcal{S}_{i} \cup \mathcal{P}_{i}$. Thus by Proposition 4.2 (i), $\mathcal{S}_{i} \cup \mathcal{P}_{i}$ and $\mathcal{S}_{i+1} \cup \mathcal{P}_{i+1}$ are reduction equivalent. Hence, by $\mathcal{P}_{i+1}=\mathcal{P}_{i}$, the claim follows.

Thus by induction on $n$, it follows that $\mathcal{R}$ and $\mathcal{S}_{n} \cup \mathcal{P}_{n}$ are reduction equivalent. Hence if $\mathcal{S}_{n} \cup \mathcal{P}_{n}$ is confluent, $\mathcal{R}$ is confluent by Proposition 4.2 (ii).

Example 4.6 (derivations in abstract reduction-preserving completion procedure). The confluence proof of Example 4.3 is derived by the abstract reduction-preserving completion procedure. Give $\mathcal{R}_{2}=\left\{\left(\operatorname{add}_{1}\right),\left(\operatorname{add}_{2}\right),(C),(A)\right\}$ as the input to the procedure. Let us consider the following derivation.

$$
\begin{aligned}
& \left\langle\left\{\left(\operatorname{add}_{1}\right),\left(\operatorname{add}_{2}\right),(C),(A)\right\}, \emptyset\right\rangle \sim^{p}\left\langle\left\{\left(\operatorname{add}_{1}\right),\left(\operatorname{add}_{2}\right)\right\},\{(C),(A)\}\right\rangle \\
& \sim^{a}\left\langle\left\{\left(\operatorname{add}_{1}\right),\left(\operatorname{add}_{2}\right),\left(\operatorname{add}_{3}\right)\right\},\{(C),(A)\}\right\rangle \\
& \sim^{a}\left\langle\left\{\left(\operatorname{add}_{1}\right),\left(\operatorname{add}_{2}\right),\left(\operatorname{add}_{3}\right),\left(\operatorname{add}_{4}^{\prime}\right)\right\},\{(C),(A)\}\right\rangle \\
& \sim^{r}\left\langle\left\{\left(\operatorname{add}_{1}\right),\left(\operatorname{add}_{2}\right),\left(\operatorname{add}_{3}\right),\left(\operatorname{add}_{4}\right)\right\},\{(C),(A)\}\right\rangle
\end{aligned}
$$

where $\left(\operatorname{add}_{4}^{\prime}\right):+(x, \mathrm{~s}(y)) \rightarrow \mathrm{s}(+(y, x))$. As we have already demonstrated in Example 3.31. $\left\{\left(\operatorname{add}_{1}\right),\left(\operatorname{add}_{2}\right),\left(\operatorname{add}_{3}\right),\left(\operatorname{add}_{4}\right),(A),(C)\right\}=\mathcal{R}_{3}$ is confluent. Thus, by Theorem 4.5, $\mathcal{R}_{2}$ is confluent.

4.2. Concrete completion procedure. In this subsection, we present a concrete reductionpreserving completion procedure that can be used as the basis of an automated completion procedure. The procedure presented below is designed so as to apply Theorem 3.18, but it is straightforward to modify the procedure suitable for Theorems 3.8, 3.28 or Corollaries $3.10,3.19,3.29$ or any combinations of them. 
Definition 4.7 (concrete reduction-preserving completion procedure).

Input: a TRS $\mathcal{R}$

Output: Success or Failure (or may diverge)

Step 1 Put $\mathcal{Q}_{0}:=\mathcal{R}$ and $i:=0$. Proceed to Step 2.

Step 2 Take a partition $\mathcal{S}_{i} \cup \mathcal{P}_{i}=\mathcal{Q}_{i}$ such that $\mathcal{S}_{i}$ is left-linear and terminating, $\mathcal{P}_{i}$ is reversible and $\mathrm{CP}_{i n}\left(\mathcal{P}_{i} \cup \mathcal{P}_{i}^{-1}, \mathcal{S}_{i}\right)=\emptyset$. Proceed to Step 3. If there is no such a partition then return Failure.

Step 3 Set $\mathcal{U}_{1}:=\emptyset$.

For each $\langle u, v\rangle \in \operatorname{CP}\left(\mathcal{S}_{i}, \mathcal{P}_{i} \cup \mathcal{P}_{i}^{-1}\right)$, do:

- Take $\mathcal{S}_{i}$-normal forms $\hat{u}, \hat{v}$ of $u, v$, respectively.

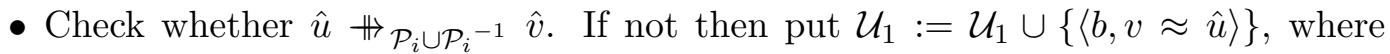
$b:=$ true or $:=$ false according to whether $v$ is $\mathcal{S}_{i}$-normal or not.

Step 4 Set $\mathcal{U}_{2}:=\emptyset$.

For each $\langle u, v\rangle \in \operatorname{CP}\left(\mathcal{S}_{i}, \mathcal{S}_{i}\right)$, do:

- Take $\mathcal{S}_{i}$-normal forms $\hat{u}, \hat{v}$ of $u, v$, respectively.

- Check whether $\hat{u} \mathbb{H}_{\mathcal{P}_{i} \cup \mathcal{P}_{i}{ }^{-1}} \hat{v}$. If not then put $\mathcal{U}_{2}:=\mathcal{U}_{2} \cup\{\langle$ false, $\hat{u} \approx \hat{v}\rangle\}$.

If $\mathcal{U}_{1}=\mathcal{U}_{2}=\emptyset$ then return Success.

Step 5 Let $\mathcal{P}^{\prime}:=\emptyset$.

For each $\langle b, u \approx v\rangle \in \mathcal{U}_{1} \cup \mathcal{U}_{2}$, do:

- Check whether there exist $u^{\prime}, v^{\prime}$ such that $u=u^{\prime} \mathbb{H}_{\mathcal{P}_{i} \cup \mathcal{P}_{i}{ }^{-1}} v^{\prime} \stackrel{*}{\leftarrow} \mathcal{S}_{i} \cup \mathcal{P}_{i} \cup \mathcal{P}_{i}{ }^{-1} v$ if

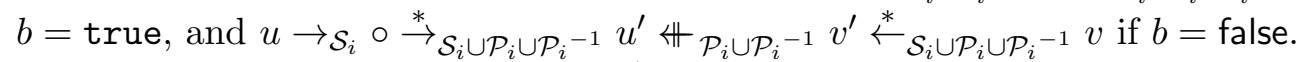

- Put $\mathcal{P}^{\prime}:=\mathcal{P}^{\prime} \cup\left\{l \rightarrow r \in \mathcal{P}_{i} \cup \mathcal{P}_{i}{ }^{-1} \mid l \rightarrow r\right.$ is used in the rewrite sequences $u \stackrel{*}{\rightarrow} \mathcal{S}_{i} \cup \mathcal{P}_{i} \cup \mathcal{P}_{i}{ }^{-1} u^{\prime}$ or $\left.v \stackrel{*}{\rightarrow} \mathcal{S}_{i} \cup \mathcal{P}_{i} \cup \mathcal{P}_{i}{ }^{-1} v^{\prime}\right\}$.

Finally, check whether $\mathcal{S}_{i}$ is terminating relative to $\mathcal{P}^{\prime}$. If it is, then return Success. Otherwise take some set $\mathcal{U}^{\prime} \subseteq\left\{v \rightarrow \hat{u} \mid\langle b, v \approx \hat{u}\rangle \in \mathcal{U}_{1}\right\} \cup\{l \rightarrow r, r \rightarrow l \mid\langle b, l \approx r\rangle \in$ $\left.\mathcal{U}_{2}, l \stackrel{*}{\leftrightarrow} \mathcal{P}_{i} r\right\}$ of rewrite rules and put $\mathcal{Q}_{i+1}:=\mathcal{Q}_{i} \cup \mathcal{U}^{\prime}, i:=i+1$ and go to Step 2.

During the step 2, one may perform the following additional steps.

Step 2a. If there exist $l \rightarrow r \in \mathcal{S}_{i}$ and $r^{\prime}$ such that $r \leftrightarrow_{\mathcal{P}_{i}} r^{\prime}$ and $\mathrm{CP}_{i n}\left(\mathcal{P}_{i} \cup \mathcal{P}_{i}{ }^{-1},\{l \rightarrow r\}\right) \neq \emptyset$, then put $\mathcal{Q}_{i+1}:=\left(\mathcal{Q}_{i} \backslash\{l \rightarrow r\}\right) \cup\left\{l \rightarrow r^{\prime}\right\}, i:=i+1$.

Step 2b. Let $\langle u, v\rangle \in \mathrm{CP}_{i n}\left(\mathcal{P}_{i} \cup \mathcal{P}_{i}^{-1}, \mathcal{S}_{i}\right)$ and let $\hat{v}$ be an $\mathcal{S}_{i}$-normal form of $v$. Then put $\mathcal{Q}_{i+1}:=\mathcal{Q}_{i} \cup\{u \rightarrow \hat{v}\}$ and $i:=i+1$.

Before moving from step 4 to step 2, one may perform the following additional step.

Step 4a. Set $\mathcal{S}_{i}:=\mathcal{S}_{i-1}, \mathcal{P}_{i}:=\mathcal{P}_{i-1}$. If there exist $l \rightarrow r \in \mathcal{S}_{i}$ and $r^{\prime}$ such that $r \leftrightarrow \mathcal{P}_{i} r^{\prime}$ and there exists $\langle u, v\rangle \in \mathrm{CP}\left(\{l \rightarrow r\}, \mathcal{P}_{i} \cup \mathcal{P}_{i}{ }^{-1}\right) \cup \mathrm{CP}\left(\{l \rightarrow r\}, \mathcal{S}_{i}\right) \cup \mathrm{CP}\left(\mathcal{S}_{i},\{l \rightarrow r\}\right)$ such that the critical pair conditions are not satisfied, then put $\mathcal{Q}_{i+1}:=\left(\mathcal{Q}_{i} \backslash\{l \rightarrow\right.$ $r\}) \cup\left\{l \rightarrow r^{\prime}\right\}, i:=i+1$.

Remark 4.8. Steps $2-5$ try to show the condition of Theorem 3.18 directly, and if the relative termination check of $\mathcal{S}_{i}$ relative to $\mathcal{P}^{\prime}$ fails in Step 5, some of critical pairs which lead to the equations in $\mathcal{U}_{1} \cup \mathcal{U}_{2}$ are problematic. Thus, taking some non-empty $\mathcal{U}^{\prime}$ is indispensable for the success of the completion procedure. On the other hand, it is not at all guaranteed whether extra steps $2 \mathrm{a}, 2 \mathrm{~b}, 4 \mathrm{a}$ are helpful for the success of the completion procedure - they just add some flexibilities to modify equations. Adding such flexibilities may be helpful but there is a trade-off between adding such extra steps and efficiency of 
the completion procedure. We here present these extra steps because they perform well for some examples, including Example 4.11 below.

Corollary 4.9 (soundness of the concrete reduction-preserving completion procedure). If the procedure of Definition 4.7 succeeds for the input $\mathcal{R}$, then $\mathcal{R}$ is confluent.

Proof. It suffices to show if the procedure succeeds then there exists a successful derivation of the abstract reduction-preserving completion procedure ending with $\mathcal{S}_{n}, \mathcal{P}_{n}$ satisfying the conditions of Theorem 3.18 . Step 1 corresponds to the empty derivation. Step 2 corresponds to an inference step by Partition. If the procedure succeeds at Step 4 , then $\mathcal{S}_{i}, \mathcal{P}_{i}$ satisfy the conditions of Corollary 3.19 and hence that of Theorem 3.18. If the procedure succeeds at Step 5, then $\mathcal{S}_{i}, \mathcal{P}_{i}$ satisfy the conditions of Theorem 3.18. Suppose that the procedure does not return Success at Steps 4 or 5 . Then it is readily checked that for $l \rightarrow r \in \mathcal{U}^{\prime}$, $l \leftarrow \mathcal{S}_{i} \circ \rightarrow_{\mathcal{P}_{i} \cup \mathcal{P}_{i}^{-1}} r$ or $l \stackrel{*}{\leftrightarrow} \mathcal{P}_{i} r$ holds. Thus, in this case, Step 5 is simulated by multiple inference steps by Addition. Similarly, Step $2 \mathrm{~b}$ is simulated by multiple inference steps by Addition and Steps $2 \mathrm{a}$ and $4 \mathrm{a}$ are simulated by inference steps by Replace.

Example 4.10. Give $\mathcal{R}_{2}=\left\{\left(\operatorname{add}_{1}\right),\left(\operatorname{add}_{2}\right),(C),(A)\right\}$ as the input.

(1) (Step 1) We put $\mathcal{Q}_{0}:=\mathcal{R}_{2}$.

(2) (Step 2) We take $\mathcal{S}_{0}=\left\{\left(\operatorname{add}_{1}\right),\left(\operatorname{add}_{2}\right)\right\}$ and $\mathcal{P}_{0}=\{(C),(A)\}$. Then $\mathcal{S}_{0}$ is left-linear and terminating, $\mathcal{P}_{0}$ is reversible and $\mathrm{CP}_{\text {in }}\left(\mathcal{P}_{0} \cup \mathcal{P}_{0}{ }^{-1}, \mathcal{S}_{0}\right)=\emptyset$.

(3) (Step 3) We have $\mathrm{CP}\left(\mathcal{S}_{0}, \mathcal{P}_{0} \cup \mathcal{P}_{0}{ }^{-1}\right)=$

$$
\left\{\begin{array}{llll}
(1) & \langle+(y, z),+(0,+(y, z))\rangle & (5) & \langle\mathrm{s}(+(x,+(y, z))),+(+(\mathrm{s}(x), y), z)\rangle \\
(2) & \langle+(y, z),+(+(0, y), z)\rangle & (6) & \langle+(\mathrm{s}(+(x, y)), z),+(\mathrm{s}(x),+(y, z))\rangle \\
(3) & \langle+(x, z),+(+(x, 0), z)\rangle & (7) & \langle+(x, \mathrm{~s}(+(y, z))),+(+(x, \mathrm{~s}(y)), z)\rangle \\
(4) & \langle y,+(y, 0)\rangle & (8) & \langle\mathrm{s}(+(x, y)),+(y, \mathrm{~s}(x))\rangle
\end{array}\right\} .
$$

Then for $\langle u, v\rangle \in\{(3),(4),(7),(8)\}, u \stackrel{*}{\rightarrow} \mathcal{S}_{0} \circ \mathbb{H}_{\mathcal{P}_{0} \cup \mathcal{P}_{0}^{-1}} \circ \stackrel{*}{\leftarrow} \mathcal{S}_{0} v$ does not hold and $v$ is $\mathcal{S}_{0}$-normal. Thus we put

$$
\mathcal{U}_{1}:=\left\{\begin{array}{l}
\langle\text { true },+(+(x, 0), z) \approx+(x, z)\rangle \\
\langle\text { true },+(y, 0) \approx y\rangle \\
\langle\operatorname{true},+(+(x, \mathrm{~s}(y)), z) \approx+(x, \mathrm{~s}(+(y, z)))\rangle \\
\langle\text { true },+(y, \mathrm{~s}(x)) \approx \mathrm{s}(+(x, y))\rangle
\end{array}\right\}
$$

and proceed to Step 4.

(4) (Step 4) We have $\operatorname{CP}\left(\mathcal{S}_{0}, \mathcal{S}_{0}\right)=\emptyset$ and thus $\mathcal{U}_{2}=\emptyset$. Since $b=$ true for any $\langle b, u \approx$ $v\rangle \in \mathcal{U}_{1}$, we check $u \stackrel{\#}{\mathcal{P}_{0} \cup \mathcal{P}_{0}}{ }^{-1} \circ \stackrel{*}{\leftarrow} \mathcal{S}_{0} \cup \mathcal{P}_{0} \cup \mathcal{P}_{0}{ }^{-1} v$. But this fails since, for example, $+(y, 0) \mathbb{W}_{\mathcal{P}_{0} \cup \mathcal{P}_{0}{ }^{-1}} \circ \stackrel{*}{\leftarrow} \mathcal{S}_{0} \cup \mathcal{P}_{0} \cup \mathcal{P}_{0}{ }^{-1} y$ does not hold. Now we put

$$
\mathcal{U}^{\prime}:=\left\{\left(\operatorname{add}_{3}\right)+(y, 0) \rightarrow y \quad\left(\operatorname{add}_{4}^{\prime}\right) \quad+(y, \mathrm{~s}(x)) \rightarrow \mathrm{s}(+(x, y))\right\} .
$$

and go to the step 2 .

(5) (Step 2) We take $\mathcal{S}_{1}=\left\{\left(\operatorname{add}_{1}\right),\left(\operatorname{add}_{2}\right),\left(\operatorname{add}_{3}\right),\left(\operatorname{add}_{4}^{\prime}\right)\right\}$ and $\mathcal{P}_{1}=\{(C),(A)\}$. Then $\mathcal{S}_{1}$ is left-linear and terminating, $\mathcal{P}_{1}$ is reversible, and $\mathrm{CP}_{i n}\left(\mathcal{P}_{1} \cup \mathcal{P}_{1}^{-1}, \mathcal{S}_{1}\right)=\emptyset$. 
(6) (Step 3) There are four elements in $\mathrm{CP}^{-1}\left(\mathcal{S}_{1}, \mathcal{P}_{1} \cup \mathcal{P}_{1}^{-1}\right)$ which are not joinable as $u \stackrel{*}{\rightarrow} \mathcal{S}_{1} \circ \mathbb{H}_{\mathcal{P}_{1} \cup \mathcal{P}_{1}^{-1}} \circ \stackrel{*}{\leftarrow} \mathcal{S}_{1} v$, namely

$$
\left\{\begin{array}{cc}
(9) & \langle+(x, \mathrm{~s}(+(y, z)),+(+(x, \mathrm{~s}(y)), z)\rangle \\
(10) & \langle\mathrm{s}(+(x,+(y, z)),+(y,+(z, \mathrm{~s}(x)))\rangle \\
(11) & \langle+(\mathrm{s}(+(x, y)), z),+(y,+(\mathrm{s}(x), z))\rangle \\
(12) & \langle+(x, \mathrm{~s}(+(y, z))),+(+(x, z), \mathrm{s}(y))\rangle
\end{array}\right\}
$$

We put

$$
\mathcal{U}_{1}:=\left\{\begin{array}{l}
\langle\text { false }+(+(x, \mathrm{~s}(y)), z) \approx \mathrm{s}(+(x,+(y, z)))\rangle \\
\langle\mathrm{false},+(y,+(z, \mathrm{~s}(x))) \approx \mathrm{s}(+(x,+(y, z))\rangle \\
\langle\mathrm{false},+(y,+(\mathrm{s}(x), z)) \approx \mathrm{s}(+(+(x, y), z)\rangle \\
\langle\mathrm{false},+(+(x, z), \mathrm{s}(y)) \approx \mathrm{s}(+(x,+(y, z)))\rangle
\end{array}\right\}
$$

and proceed to Step 4.

(7) (Step 4) We have $\operatorname{CP}\left(\mathcal{S}_{1}, \mathcal{S}_{1}\right)=\emptyset$ and thus $\mathcal{U}_{2}=\emptyset$. Since $b=$ false for any $\langle b, u \approx$

$v\rangle \in \mathcal{U}_{1}$, we check $u \rightarrow \mathcal{S}_{1} \circ \stackrel{*}{\rightarrow} \mathcal{S}_{1} \cup \mathcal{P}_{1} \cup \mathcal{P}_{1}-1 \quad u^{\prime} \mathbb{H}_{\mathcal{P}_{1} \cup \mathcal{P}_{1}-1} v^{\prime} \stackrel{*}{\leftarrow} \mathcal{S}_{1} \cup \mathcal{P}_{1} \cup \mathcal{P}_{1}-1 \quad v$. Then we have $u \rightarrow \mathcal{S}_{1} \circ \stackrel{*}{\rightarrow}_{\mathcal{S}_{1} \cup \mathcal{P}^{\prime}} u^{\prime} \mathbb{H}_{\mathcal{P}_{1} \cup \mathcal{P}_{1}-1} v^{\prime} \stackrel{*}{\leftarrow} \mathcal{S}_{1} \cup \mathcal{P}^{\prime} v$ for all $\langle b, u \approx v\rangle \in \mathcal{U}_{1}$ by taking $\mathcal{P}^{\prime}=\{+(+(x, y), z) \rightarrow+(x,+(y, z))\}$. Then $\mathcal{S}_{1}$ is terminating relative to $\mathcal{P}^{\prime}$ and Success is returned.

Example 4.11. Suppose that the relative termination check of $\mathcal{S}_{1}$ relative to $\mathcal{P}^{\prime}$ fails in the last step of Example 4.10. Then the procedure continues as follows.

(8) Here, we put $\mathcal{U}^{\prime}:=\emptyset, \mathcal{Q}_{2}:=\mathcal{Q}_{1}, i:=2$ and proceed to Step 4a.

(9) (Step 4a) Put $\mathcal{S}_{2}:=\mathcal{S}_{1}$ and $\mathcal{P}_{2}:=\mathcal{P}_{1}$. Since (11) $\in \mathrm{CP}\left(\left\{\left(\operatorname{add}_{4}^{\prime}\right)\right\}, \mathcal{P}_{2} \cup \mathcal{P}_{2}{ }^{-1}\right)$ and $\mathrm{s}(+(x, y)) \rightarrow_{\mathcal{P}_{2}} \mathrm{~s}(+(y, x))$, one can put $\left.\mathcal{Q}_{3}:=\left(\mathcal{Q}_{2} \backslash\left\{\left(\operatorname{add}_{4}^{\prime}\right)\right\}\right) \cup\left(\operatorname{add}_{4}\right)\right\}$ and $i:=3$ and go to Step 2.

(10) (Step 2) We take $\mathcal{S}_{3}=\left\{\left(\operatorname{add}_{1}\right),\left(\operatorname{add}_{2}\right),\left(\operatorname{add}_{3}\right),\left(\operatorname{add}_{4}\right)\right\}$ and $\mathcal{P}_{3}=\{(C),(A)\}$. Then $\mathcal{S}_{3}$ is left-linear and terminating, $\mathcal{P}_{3}$ is reversible and $\mathrm{CP}_{\text {in }}\left(\mathcal{P}_{3} \cup \mathcal{P}_{3}{ }^{-1}, \mathcal{S}_{3}\right)=\emptyset$. Thus proceed to Step 3.

(11) (Step 3) For any $\langle u, v\rangle \in \mathrm{CP}\left(\mathcal{S}_{3}, \mathcal{P}_{3} \cup \mathcal{P}_{3}{ }^{-1}\right)$, we have $u \stackrel{*}{\rightarrow} \mathcal{S}_{3} \circ \mathbb{H}_{\mathcal{P}_{3} \cup \mathcal{P}_{3}^{-1}} \circ \stackrel{*}{\leftarrow} \mathcal{S}_{3} v$ (Example 3.31). Thus $\mathcal{U}_{1}:=\emptyset$ and proceed to Step 4.

(12) (Step 4) For any $\langle u, v\rangle \in \mathrm{CP}\left(\mathcal{S}_{3}, \mathcal{S}_{3}\right)$, we have $u \stackrel{*}{\rightarrow}_{\mathcal{S}_{3}} \circ \mathbb{H}_{\mathcal{P}_{3} \cup \mathcal{P}_{3}^{-1}} \circ \stackrel{*}{\leftarrow}_{\mathcal{S}_{3}} v$ (Example 3.31). Thus $\mathcal{U}_{2}:=\emptyset$. Since $\mathcal{U}_{1}=\mathcal{U}_{2}=\emptyset$, success is returned.

\section{IMPLEMENTATION AND EXPERIMENTS}

All the results of this paper have been implemented. The program is written in SML/N $\sqrt{2}$ and is built upon our confluence prover $\mathrm{ACP}^{3}[1,3,27$.

In Figure 3, we present pseudo-code of main functions of our implementation of reductionpreserving completion procedure enough for describing some heuristics employed in the implementation. Short descriptions of functions involved in our pseudo-code and heuristics employed follow.

- (checkConfluence $\mathcal{R}$ ) is the main function of the procedure. It simulates multiple runs in the breadth-first strategy.

\footnotetext{
$2_{\text {http: //www.smlnj.org/ }}$

$3_{\text {http: //www.nue.riec.tohoku.ac.jp/tools/acp/ }}$
} 


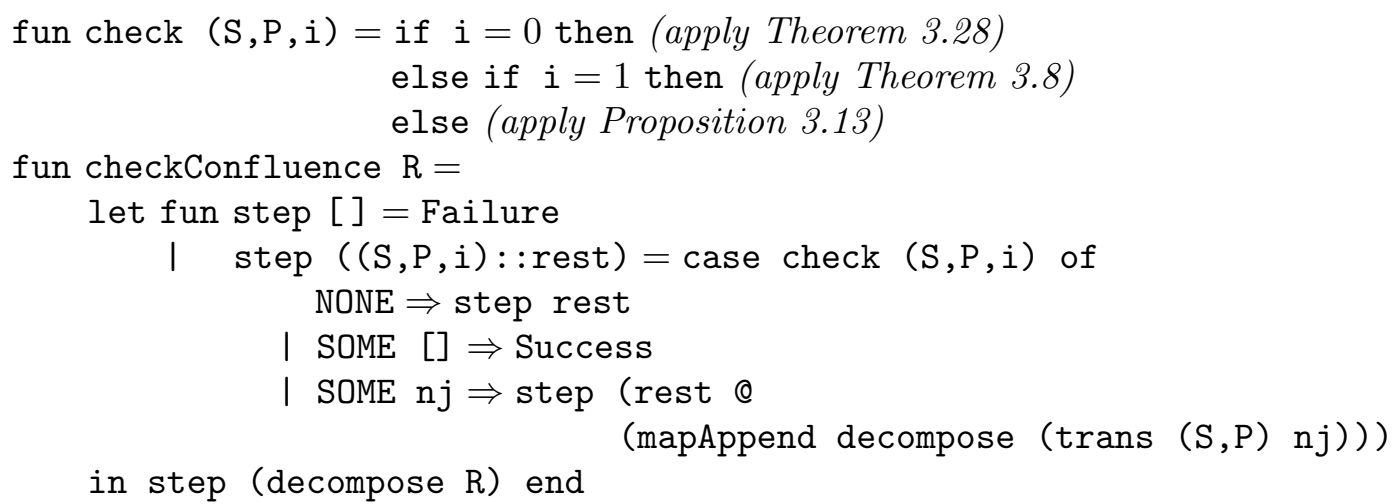

Figure 3: Pseudo-code of main functions

- (decompose $\mathcal{R}$ ) decomposes $\mathcal{R}$ into $\mathcal{S} \cup \mathcal{P}$ and duplicates $\mathcal{S} \cup \mathcal{P}$. Hence a list of triples $(\mathcal{S}, \mathcal{P}, i)$ where $\mathcal{S} \cup \mathcal{P}=\mathcal{R}$ and $i \in\{0,1,2\}$ are returned. Here, however, instead of returning all partitions, we select partitions based on a heuristic, namely that $\mathcal{P}$ is a set of the rules $l \rightarrow r$ satisfying either (1) $r \rightarrow l \in \mathcal{R}$ or $(2) \mathcal{F}(l)=\mathcal{F}(r)$ and $l(\epsilon)=r(\epsilon)$.

- $(\operatorname{check}(\mathcal{S}, \mathcal{P}, i))$ checks whether conditions of Theorem 3.8 when $i=0$ (or Theorem 3.28 when $i=1$, Proposition 3.13 when $i=2$ ) are satisfied based on the algorithm given in our concrete completion procedure (Definition 4.7). Reversibility is tested by checking $r \stackrel{\leq k}{\rightarrow} l$ (i.e. there is a rewrite sequence from $r$ to $l$ of length less than or equal to $k$ steps) for some constant $k$. In our implementation, we set $k=10$.

- (trans $(\mathcal{S}, \mathcal{P}) \mathrm{nj}$ ) returns a collection of transformed TRSs obtained by addition and replacement of rewrite rules constructed from non-joinable critical pairs (Steps 5 and 2b of the Definition 4.7) and rewrite rules generating such critical pairs (Steps 2a and 4a of of the Definition 4.7). Here, the addition of rewrite rules is restricted based on some heuristic.

Table 1 shows the summary of our experiments. We have tested various combinations of our results: Rows (1)-(8) are proofs by confluence criteria of Theorems 3.8, 3.18, 3.28 (Corollaries 3.10, 3.19, 3.29) and by the combination of those of Theorem 3.28 (Corollary 3.29 and Theorem 3.8 (Corollary 3.10, respectively). Those marked with "w/o RT" are the ones without (proper) relative termination checking (i.e. Corollaries 3.10, 3.19 and 3.29 where only termination checking is involved). Rows (9)-(14) are proofs by the reduction-preserving completion without the Replacement rule, i.e. the Steps $2 a, 4 a$ of the concrete reduction-preserving completion (Definition 4.7). Rows (15)-(20) are proofs by the reduction-preserving completion with the Replacement rule. Row (21) are proofs by the reduction-preserving completion with the Replacement rule and Huet's criterion (Proposition 3.13.

For the experiments, we used a collection of 85 TRSs involving non-terminating rules such as commutativity and associativity rules which have been developed in the course of experiment: 4 All experiments have been performed on a Linux platform of a PC equipped

\footnotetext{
${ }^{4}$ Four TRSs have been added to the collection of our proceedings version 2] of the paper to capture Examples 3.35 and 3.37
} 
with $1.2 \mathrm{GHz}$ CPU and $1 \mathrm{~GB}$ memory. The maximal number of steps of the completion procedure is limited to 20 steps; the columns below the title "diverge" show the numbers of examples which exceeded this limit, where these numbers are included in those of "failure." We set the timeout $60 \mathrm{sec}$. The columns below the title "timeout" show the numbers of examples which exceeded this timeout. Total time (real time) is measured in seconds.

When relative termination checking is disabled and only termination checking is used, the applicability of incomparable confluent criteria (Corollary 3.29 and Corollary 3.10) does not show much differences in the number of success. When relative termination checking is activated, Theorem 3.28 has a clear advantage over Theorem 3.8 and at the same time total computation time rises much - this is because of relative termination checking invoked multiple times for solving each problem. The applicability of Theorem 3.18 (Corollary 3.19) which is subsumed by Theorem 3.28 (Corollary 3.29, respectively) is limited compared to these two criteria. Comparing to Huet's criterion (Proposition 3.13) which succeeds at 37 examples, the direct application of the combination of Theorems 3.8 and 3.28 without reduction-preserving completion succeeded at 41 examples.

In each criterion, there is a clear increase of the number of success by adding the completion procedure. The increase of total time by the introduction of completion procedure is not much but this depends on largely our heuristics of choosing partitions and the limitation on the number of completion steps. The number of successful examples, however, does not change even when we increase that limit to 150 steps. Activation of relative termination checking is also effective even in the presence of completion procedure. The introduction of the Replacement inference rule only makes a difference when relative termination checking is not involved.

We have also tested the confluence prover ACP on our collection. ACP is an automated confluence prover in which divide-and-conquer approach based on the persistent, layerpreserving, commutative decompositions is employed and involving many confluence criteria [6, 8, 16, 17, 20, 22, 23, 18, 25] as well as the decreasing diagram techniques [24, 26]. As shown in the table, most of our examples are not coped with by the confluence prover ACP.

The collection of examples and all details of the experiments are available on the webpage http://www.nue.riec.tohoku.ac.jp/tools/acp/experiments/12lmcs/all.html.

\section{Conclusion}

We have presented a new method for proving confluence of TRSs. The scope of our method is a class of TRSs that can be partitioned into a terminating part and a reversible part. Our method is applicable for TRSs containing non-terminating rules such as commutativity and associativity which have been difficult to deal with most of the standard methods.

We have given a new abstract criterion for Church-Rosser modulo (Theorem 2.2) which extends those that appeared in [8] and [9]. Based on this abstract criterion, we have given two new criteria for confluence of TRSs formulated in terms of its terminating part $\mathcal{S}$ and its reversible part $\mathcal{P}$ (Theorems 3.8 and 3.28). These criteria are effective even if $\mathcal{S}$ is not terminating relative to $\mathcal{P}$; in case $\mathcal{S}$ is terminating relative to a part of $\mathcal{P}$ then the applicability of criteria is strengthened. We have also given a reduction-preserving completion by which applicability of our criteria is enhanced. We have implemented the proposed techniques and reported experimental results. 
Table 1: Summary of experiments

\begin{tabular}{l|c|c|c|c|r}
\hline & success & failure & diverge & timeout & time(sec.) \\
\hline (1) linear (w/o RT) (Cor. 3.10) & 28 & 57 & 0 & 0 & 7.28 \\
(2) linear (Thm. 3.8) & 32 & 53 & 0 & 0 & 7.41 \\
(3) parallel (w/o RT) (Cor. 3.19) & 19 & 66 & 0 & 0 & 7.93 \\
(4) parallel (Thm. 3.18) & 24 & 61 & 0 & 0 & 8.20 \\
(5) PCP (w/o RT) (Cor. 3.29) & 28 & 57 & 0 & 0 & 7.97 \\
(6) PCP (Thm. 3.28) & 39 & 46 & 0 & 0 & 21.44 \\
(7) linear\&PCP (w/o RT) (Cor. 3.10\&3.29) & 30 & 55 & 0 & 0 & 8.82 \\
(8) linear\&PCP (Thm. 3.8\&/3.28) & 41 & 44 & 0 & 0 & 21.28 \\
(9) completion (linear,w/o RT) & 47 & 38 & 0 & 0 & 8.17 \\
(10) completion (linear) & 61 & 24 & 0 & 0 & 9.00 \\
(11) completion (PCP,w/o RT) & 50 & 35 & 0 & 0 & 9.85 \\
(12) completion (PCP) & 74 & 11 & 0 & 0 & 30.18 \\
(13) completion (linear\&PCP,w/o RT) & 52 & 33 & 0 & 0 & 10.84 \\
(14) completion (linear\&PCP) & 77 & 8 & 0 & 0 & 28.26 \\
(15) completion+repl (linear,w/o RT) & 61 & 24 & 0 & 0 & 9.51 \\
(16) completion+repl (linear) & 61 & 24 & 0 & 0 & 9.09 \\
(17) completion+repl (PCP,w/o RT) & 66 & 19 & $(3)$ & 0 & 14.90 \\
(18) completion+repl (PCP) & 75 & 10 & 0 & 0 & 29.49 \\
(19) completion+repl (linear\&PCP,w/o RT) & 69 & 16 & $(2)$ & 0 & 14.61 \\
(20) completion+repl (linear\&PCP) & 77 & 8 & 0 & 0 & 29.05 \\
(21) (20) + Huet (Prop. 3.13) & 77 & 8 & 0 & 0 & 40.95 \\
\hline Huet (Prop. 3.13) & 37 & 48 & 0 & 0 & 31.22 \\
ACP [1, 3, 27 & 13 & 72 & - & $(2)$ & 196.40 \\
\hline
\end{tabular}

\section{ACKNOWLEDGMENT}

Thanks are due to Junichi Mitimata for discussions and experiments on preliminary results of this paper. The authors are grateful for Harald Zankl, Aart Middeldorp, Dominik Klein for pointers to related works and/or helpful comments. Thanks are due to anonymous referees for detailed comments. This work was partially supported by grants from JSPS Nos. 20500002 and 22500002.

\section{REFERENCES}

[1] T. Aoto. Automated confluence proof by decreasing diagrams based on rule-labelling. In C. Lynch, editor, Proc. of RTA 2010, volume 6 of LIPIcs, pages 7-16. Schloss Dagstuhl, 2010.

[2] T. Aoto and Y. Toyama. A reduction-preserving completion for proving confluence of non-terminating term rewriting systems. In M. Schmidt-Schauß, editor, Proc. of RTA 2011, volume 10 of LIPIcs, pages 91-106. Schloss Dagstuhl, 2011.

[3] T. Aoto, Y. Yoshida, and Y. Toyama. Proving confluence of term rewriting systems automatically. In R. Treinen, editor, Proc. of RTA 2009, volume 5595 of LNCS, pages 93-102. Springer-Verlag, 2009.

[4] F. Baader and T. Nipkow. Term Rewriting and All That. Cambridge University Press, 1998.

[5] L. Bachmair and N. Dershowitz. Completion for rewriting modulo a congruence. Theoretical Computer Science, 67(2-3):173-201, 1981.

[6] H. Gomi, M. Oyamaguchi, and Y. Ohta. On the Church-Rosser property of root-E-overlapping and strongly depth-preserving term rewriting systems. Transactions of IPSJ, 39(4):992-1005, 1998. 
[7] B. Gramlich. Confluence without termination via parallel critical pairs. In H. Kirchner, editor, Proc. of CAAP'96, volume 1996 of LNCS, pages 211-225. Springer-Verlag, 2006.

[8] G. Huet. Confluent reductions: abstract properties and applications to term rewriting systems. Journal of the ACM, 27(4):797-821, 1980.

[9] J.-P. Jouannaud and H. Kirchner. Completion of a set of rules modulo a set of equations. SIAM Journal of Computing, 15(4):1155-1194, 1986.

[10] J.-P. Jouannaud, H. Kirchner, and J. L. Remy. Church-Rosser properties of weakly terminating equational term rewriting systems. In A. Bundy, editor, Proc. of 8th IJCAI, pages 909-915, 1983.

[11] D. S. Lankford and A. M. Ballantyne. Decision procedures for simple equational theories with commutative-associative axioms: complete sets of commutative-associative reductions. Technical Report ATP-39, Department of Computer Sciences, University of Texas at Austin, 1977.

[12] D. S. Lankford and A. M. Ballantyne. Decision procedures for simple equational theories with commutative axioms: complete sets of commutative reductions. Technical Report ATP-35, Department of Computer Sciences, University of Texas at Austin, 1977.

[13] D. S. Lankford and A. M. Ballantyne. Decision procedures for simple equational theories with permutative axioms: complete sets of permutative reductions. Technical Report ATP-37, Department of Computer Sciences, University of Texas at Austin, 1977.

[14] A. Middeldorp and M. Starčević. A rewrite approach to polynomial ideal theory. Report CS-R9160, CWI, Amsterdam, 1991.

[15] E. Ohlebusch. Church-Rosser theorems for abstract reduction modulo an equivalence relation. In T. Nipkow, editor, Proc. of RTA-98, volume 1379 of LNCS, pages 17-31. Springer-Verlag, 1998.

[16] S. Okui. Simultaneous critical pairs and Church-Rosser property. In T. Nipkow, editor, Proc. of RTA-98, volume 1379 of $L N C S$, pages 2-16. Springer-Verlag, 1998.

[17] M. Oyamaguchi and Y. Ohta. A new parallel closed condition for Church-Rosser of left-linear TRS's. In H. Comon, editor, Proc. of RTA-97, volume 1232 of LNCS, pages 187-201. Springer-Verlag, 1997.

[18] M. Oyamaguchi and Y. Ohta. On the open problems concerning Church-Rosser of left-linear term rewriting systems. IEICE Trans. Information and Systems, E87-D(2):290-298, 2004.

[19] G. E. Peterson and M. E. Stickel. Complete sets of reductions for some equational theories. Journal of the ACM, 28(2):233-264, 1981.

[20] Y. Toyama. On the Church-Rosser property of term rewriting systems. Technical Report 17672, NTT ECL, 1981.

[21] Y. Toyama. Confluent term rewriting systems (invited talk). In J. Giesl, editor, Proc. of RTA 2005, volume 3467 of $L N C S$, page 1. Springer-Verlag, 2005. Slides are available from http://www.nue.riec. tohoku.ac.jp/user/toyama/slides/toyama-RTA05.pdf.

[22] Y. Toyama and M. Oyamaguchi. Church-Rosser property and unique normal form property of nonduplicting term rewriting systems. In N. Dershowitz and N. Lindenstrauss, editors, Proc. of CTRS-94, volume 968 of $L N C S$, pages 316-331. Springer-Verlag, 1994.

[23] Y. Toyama and M. Oyamaguchi. Conditional linearization of non-duplicating term rewriting systems. IEICE Trans. Information and Systems, E84-D(5):439-447, 2001.

[24] V. van Oostrom. Confluence by decreasing diagrams. Theoretical Computer Science, 126(2):259-280, 1994.

[25] V. van Oostrom. Developing developments. Theoretical Computer Science, 175(1):159-181, 1997.

[26] V. van Oostrom. Confluence by decreasing diagrams: converted. In A. Voronkov, editor, Proc. of RTA 2008, volume 5117 of LNCS, pages 306-320. Springer-Verlag, 2008.

[27] J. Yoshida, T. Aoto, and Y. Toyama. Automating confluence check of term rewriting systems. Computer Software, 26(2):76-92, 2009. In Japanese.

This work is licensed under the Creative Commons Attribution-NoDerivs License. To view a copy of this license, visit http://creativecommons.org/licenses/by-nd/2.0/ or send a letter to Creative Commons, 171 Second St, Suite 300, San Francisco, CA 94105, USA, or Eisenacher Strasse 2, 10777 Berlin, Germany 